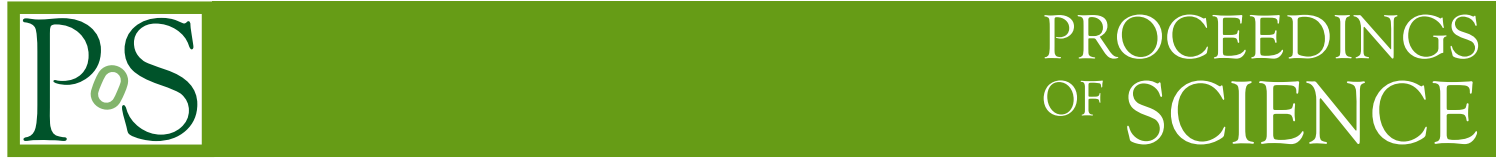

\title{
Lectures on Cosmic Inflation and its Potential Stringy Realizations
}

\section{C.P. Burgess}

Department of Physics \& Astronomy, McMaster University, 1280 Main Street West, Hamilton, Ontario, Canada, L8S $4 M 1$.

Perimeter Institute for Theoretical Physics,

31 Caroline Street North, Waterloo, Ontario, Canada, N2L $2 Y 5$.

These notes present a brief introduction to Hot Big Bang cosmology and Cosmic Inflation, together with a selection of some recent attempts to embed inflation into string theory. They provide a partial description of lectures presented in courses at Dubrovnik in August 2006, at CERN in January 2007 and at Cargèse in August 2007. They are aimed at graduate students with a working knowledge of quantum field theory, but who are unfamiliar with the details of cosmology or of string theory.

School on Particle Physics, Gravity and Cosmology

21 August - 2 September 2006

Dubrovnik, Croatia

School on Cosmology and Particle Physics Beyond the Standard Models

30 July - 11 August 2007

Cargèse, France 
The Hot Big Bang model of cosmology has recently been tested with unprecedented redundancy and precision, and has emerged all the stronger for having done so. The redundancy of these tests gives confidence that the basic picture - the expansion of an initial hot primordial soup is basically right. Their precision allows a detailed inference of the model's parameters, including the first-ever survey of the energy content of the Universe as a whole.

Although the Big Bang works well, it does so only provided that the Universe is started off in a particular way. The theory of Cosmic Inflation [1] was invented in order to try to explain these initial conditions, by postulating a much earlier epoch during which the Universe expanded increasingly rapidly with time. Remarkably, this proposal turns out also to give a good explanation for the properties of the temperature fluctuations that were later seen in the Cosmic Microwave Background Radiation (CMBR) - the residual radiation left over from the first epoch when the universe became transparent to photons, due to its cooling enough to allow ordinary matter to become dominated by neutral atoms.

Because inflation likely takes place at temperatures much higher than any ever seen in the lab on Earth, its study necessarily involves making assumptions about what kinds of physics are involved at such high energies. This, together with the observational successes, has stimulated a variety of attempts to try to find inflationary configurations within string theory, which remains our best candidate for the physics relevant to such high energies. These notes are meant as a brief introduction to inflationary cosmology and its potential stringy realizations, aimed at an audience of graduate students in particle physics.

\section{Hot Big Bang Cosmology}

We start with a description of the geometry of spacetime on which all of the subsequent sections rely, together with a telegraphic summary of the essentials of the Hot Big Bang model. (More details can be found in one of the following excellent books [2,3].) The key underlying assumption in this section is that the universe is homogeneous and isotropic when seen on the largest distance scales. Until relatively recently this assertion about the homogeneity and isotropy of the universe was an assumption, often called the Cosmological Principle. More recently it has become possible to put this assertion on an observational footing, based on large-scale surveys of the distribution of matter and radiation within the universe we see around us. Most notable among these is the incredible uniformity of the observed temperature of the CMBR, for which temperature fluctuations are observed to be of order $\delta T / T \sim 10^{-5}$.

\subsection{Friedman-Robertson-Walker Cosmology}

In General Relativity the geometry of spacetime is specified by its metric tensor, which defines the differential distance, $\mathrm{d} s^{2}=g_{\mu \nu} \mathrm{d} x^{\mu} \mathrm{d} x^{v}$, associated with infinitesimal coordinate displacements, $\mathrm{d} x^{\mu}$. The most general 4D geometry which is consistent with isotropy and homogeneity of its 
spatial slices is described by the Robertson-Walker metric:

$$
\begin{aligned}
\mathrm{d} s^{2} & =-\mathrm{d} t^{2}+a^{2}(t)\left[\frac{\mathrm{d} r^{2}}{1-\kappa r^{2}}+r^{2} \mathrm{~d} \theta^{2}+r^{2} \sin ^{2} \theta \mathrm{d} \phi^{2}\right] \\
& =-\mathrm{d} t^{2}+a^{2}(t)\left[\mathrm{d} \ell^{2}+r^{2}(\ell) \mathrm{d} \theta^{2}+r^{2}(\ell) \sin ^{2} \theta \mathrm{d} \phi^{2}\right],
\end{aligned}
$$

where $0<\theta<\pi$ and $0 \leq \phi<2 \pi$ are the usual angular coordinates on a two-sphere, and we choose ourselves to lie at the origin, $r=0$, of the radial coordinate.

Homogeneity and isotropy dictate that the 3-dimensional spatial slices through this geometry at fixed $t$ are maximally symmetric, and so are described by the three-valued quantity, $\kappa=0,1,-1$. If $\kappa=1$ then the spatial slices are three-spheres and $0<r<1$; if $\kappa=-1$ they are hyperbolic surfaces and $0<r<\infty$; and if $\kappa=0$ they are flat and again $r$ ranges from zero to infinity. The metric of eq. (1.1) follows the standard convention, wherein the freedom to redefine $r \rightarrow \lambda r$ has been used to absorb the radius of curvature of the spatial metric into the overall scale factor, $a(t)$.

The second form given for the metric in eq. (1.1) instead uses the proper distance, $\ell$, (at fixed $t$ ) as the radial coordinate, where $\mathrm{d} \ell=\mathrm{d} r /\left(1-\kappa r^{2}\right)^{1 / 2}$, and so

$$
r(\ell)=\left\{\begin{array}{ccc}
\sin \ell & \text { if } & \kappa=+1 \\
\ell & \text { if } & \kappa=0 \\
\sinh \ell & \text { if } & \kappa=-1
\end{array} .\right.
$$

Exercise 1: Find the rate of change, $V_{H}=\mathrm{d} D / \mathrm{d} t$, of the proper distance, $D=a \Delta \ell$, from us to another co-moving observer located on a galaxy at fixed position $(\ell, \theta, \phi)$. Show that this is given by the Hubble Law: $V_{H}=H D$, where $H=\dot{a} / a$ defines the instantaneous Hubble parameter.

Detailed observations of many, many galaxies broadly confirm that galaxies do recede from us in a way that is consistent with the Hubble law defined in Exercise 1, with a present-day Hubble parameter of $H_{0} \sim 75 \mathrm{~km} / \mathrm{sec} / \mathrm{Mpc}$. Strictly speaking, however, the Hubble law only applies once the peculiar motion due to the gravitational influence of local matter is removed. But since the Hubble law implies that the apparent recession due to the universal expansion becomes more important for more distant galaxies, in practice peculiar velocities are an important complication only for the nearest galaxies.

Exercise 2: For the Robertson-Walker geometry show that if a photon having wavelength $\lambda_{\mathrm{em}}$ is emitted at a time $t_{\mathrm{em}}$, when $a\left(t_{\mathrm{em}}\right)=a_{\mathrm{em}}$, and is received with a wavelength $\lambda_{\mathrm{obs}}$ at a later time $t_{\mathrm{obs}}$ for which $a\left(t_{\mathrm{obs}}\right)=a_{\mathrm{obs}}$, then it experiences a redshift $z=\left(a_{\mathrm{obs}} / a_{\mathrm{em}}\right)-1$, where redshift is defined by $z \equiv\left(\lambda_{\mathrm{obs}}-\lambda_{\mathrm{em}}\right) / \lambda_{\mathrm{em}}$. Notice that this implies that universal expansion (i.e. $a_{\mathrm{obs}}>a_{\mathrm{em}}$ ) implies $z>0$, making the observed wavelength longer (more red) than the emitted one.

How the scale factor evolves with time depends on what kind of matter the universe contains, in a way which is dictated by the field equations for gravity. Assuming these are given by Einstein's General Theory of Relativity implies that this connection between spacetime geometry and 
universal energy content is given by

$$
R_{\mu v}-\frac{1}{2} R g_{\mu v}=8 \pi G T_{\mu v}
$$

where $G$ is Newton's constant, and $R=g^{\mu v} R_{\mu v}$ where $R_{\mu v}$ denotes the Ricci tensor - a particular measure of the curvature of spacetime.

The tensor $T_{\mu \nu}$ on the right-hand-side of eq. (1.3) is the energy-momentum stress tensor of the universe's matter content, which is locally conserved in the sense that $\nabla^{\mu} T_{\mu \nu}=0$. The most general form for $T_{\mu \nu}$ consistent with the homogeneity and isotropy of spacetime has the perfect-fluid form:

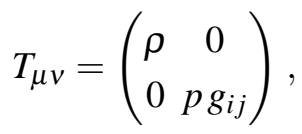

where $\rho$ is the local energy density and $p$ the local pressure. The indices $i, j=1,2,3$ run over the spatial coordinates (as opposed to the spacetime indices $\mu, v=0,1,2,3$ ).

Once eq. (1.3) is specialized to the Robertson-Walker metric, eq. (1.1), and to (1.4), it reduces to two independent equations governing the time-evolution of the scale factor, $a(t)$ : the Friedmann equation,

$$
\left(\frac{\dot{a}}{a}\right)^{2}+\frac{\kappa}{a^{2}}=\frac{\rho}{3 M_{p}^{2}} \quad \text { (Friedmann), }
$$

where $M_{p}^{-2} \equiv 8 \pi G$, and the Raychaudhuri equation,

$$
\frac{\ddot{a}}{a}=-\frac{1}{6 M_{p}^{2}}(\rho+3 p) \quad \text { (Raychaudhuri) } .
$$

It is often useful to trade eq. (1.6) for the equivalent first-order equation which expresses conservation of energy:

$$
\frac{\mathrm{d}}{\mathrm{d} t}\left(\rho a^{3}\right)=-p \frac{\mathrm{d}}{\mathrm{d} t}\left(a^{3}\right) \quad \text { (energy conservation) }
$$

since eqs. (1.5) and (1.7) together imply eq. (1.6).

\subsection{Universal energy content}

At present, the universe appears to be well-described by a fluid which contains four independent contributions to its stress energy,

$$
T_{\mu v}=\sum_{i=1}^{4} T_{\mu v}^{i} .
$$

Furthermore, each component of this fluid appears to exchange energy and momentum negligibly with the others, so $\nabla^{\mu} T_{\mu \nu}^{i}=0$, for each $i$. In terms of the corresponding energy densities, $\rho_{i}$, and pressures, $p_{i},-$ defined for $T_{\mu \nu}^{i}$ as in eq. (1.4) - this implies that each component separately satisfies eq. (1.7).

For the purposes of cosmology, several important things are known about the universal stressenergy content. 


\section{Total Energy Density:}

The best current measurements of the present-day Hubble scale, $H_{0}=(\dot{a} / a)_{0}$, together with the measured overall curvature of space, $\kappa / a_{0}^{2}$, taken with the Friedmann equation, eq. (1.5), tell us the present value of the total energy density, $\rho_{\mathrm{tot}}=\sum_{i} \rho_{i}$, of the universe. The curvature of space, $\kappa / a_{0}^{2}$, can be inferred from the properties of the measured temperature fluctuations of the CMBR together with the measured value of $H_{0}$, and imply $\kappa / a_{0}^{2}$ is presently consistent with zero (i.e. a spatially flat universe). Using this, and the measured value for $H_{0}$, in eq. (1.5) then implies

$$
\rho_{\mathrm{tot}} \sim \rho_{c}=3 M_{p}^{2} H_{0}^{2} \sim 10^{-29} \mathrm{~g} / \mathrm{cm}^{3} .
$$

The Friedmann equation, eq. (1.5), can then be rewritten as

$$
\sum_{i} \Omega_{i}=1
$$

where $\Omega_{i}=\rho_{i} / \rho_{c}$ denotes the present-day fraction of energy density contributed by each fluid component, and the sum runs over all components.

At present there is good evidence for there being the following four components to the cosmic fluid:

\section{Radiation:}

We see the universe around us is filled with photons, whose energy density is dominated by the photons of the CMBR. The pressure and energy density of a gas of photons are related by the equation of state

$$
p_{\mathrm{rad}}=\frac{1}{3} \rho_{\mathrm{rad}} .
$$

These photons are observed to have a thermal distribution, with temperature $2.715 \mathrm{~K}$.

On particle-physics grounds it is also believed that there are also an almost equally large number of Cosmic Relic Neutrinos (CRNs), whose masses are small enough to have been relativistic at least up to very recent epochs of the universe. Furthermore, these neutrinos are calculated to be thermally distributed, with temperature $T_{v} \sim 1.9 \mathrm{~K}$. Since any gas of weakly-interacting relativistic particles satisfies the equation of state, eq. (1.11), these neutrinos are normally lumped together with the photons into the energy density and pressure of cosmic radiation.

The observed total energy density of radiation is a small fraction of the present total energy density,

$$
\Omega_{\mathrm{rad}}=\left(\frac{\rho_{\mathrm{rad}}}{\rho}\right)_{\text {now }} \approx 8 \times 10^{-5}
$$

of which roughly $3 \times 10^{-5}$ comes from the neutrinos.

\section{Baryons:}

The universe also contains ordinary matter (electrons, nuclei, atoms) in large numbers, whose number density is normally counted as a contribution to the conserved density of baryon number (for which neutrons and protons carry +1 unit while electrons carry none). (Although this technically 
does not count the electrons, the overall electrical neutrality of the universe tells us that the number of electrons is the same as the number of protons.)

Since this kind of matter is non-relativistic, its average kinetic energy - i.e. its pressure - is smaller than the energy tied up in its rest mass by an amount of order $v^{2} / c^{2}$, and so its equation of state is

$$
p_{B} \approx 0 .
$$

Even though the number density of baryons is numerically much less numerous than photons, $n_{B} / n_{\gamma} \approx 5 \times 10^{-10}$, their relatively large rest mass implies they make up a larger component of the present day energy density than does the radiation:

$$
\Omega_{B}=\left(\frac{\rho_{B}}{\rho}\right)_{\text {now }} \approx 4 \%
$$

The number of visible baryons is much smaller than this, but the total amount of baryons present can nonetheless be determined because of its influence both on the observed temperature fluctuations of the CMBR and on the relative abundance of light nuclei which were formed in the very early universe.

\section{Dark Matter:}

Observations of how stars move within galaxies, how galaxies move within clusters and of how the gravity of matter as a whole influences galaxy formation and the temperature fluctuations in the CMBR provide good, consistent evidence for the existence of a large amount of non-relativistic matter which gravitates just like ordinary baryons do, also with an equation of state for nonrelativistic matter:

$$
p_{D M} \approx 0 .
$$

Agreement with observations requires the overall abundance of this Dark Matter to be

$$
\Omega_{D M}=\left(\frac{\rho_{D M}}{\rho}\right)_{\text {now }} \approx 26 \%
$$

Since both baryons and Dark Matter share the same equation of state, it is common to lump them together into an overall energy density of non-relativistic matter,

$$
\Omega_{\mathrm{m}}=\Omega_{B}+\Omega_{D M} \approx 30 \% .
$$

\section{Dark Energy:}

For the past decade evidence has been accumulating for the existence of yet another kind of invisible matter, in addition to the Dark Matter just described. The existence of this matter is inferred in two different ways.

First, it is clear that the sum of the energy density of the above-mentioned fluid components does not yet add up to the observed total energy density, $\rho_{c}$. (Fig. 1 shows the accuracy of this 

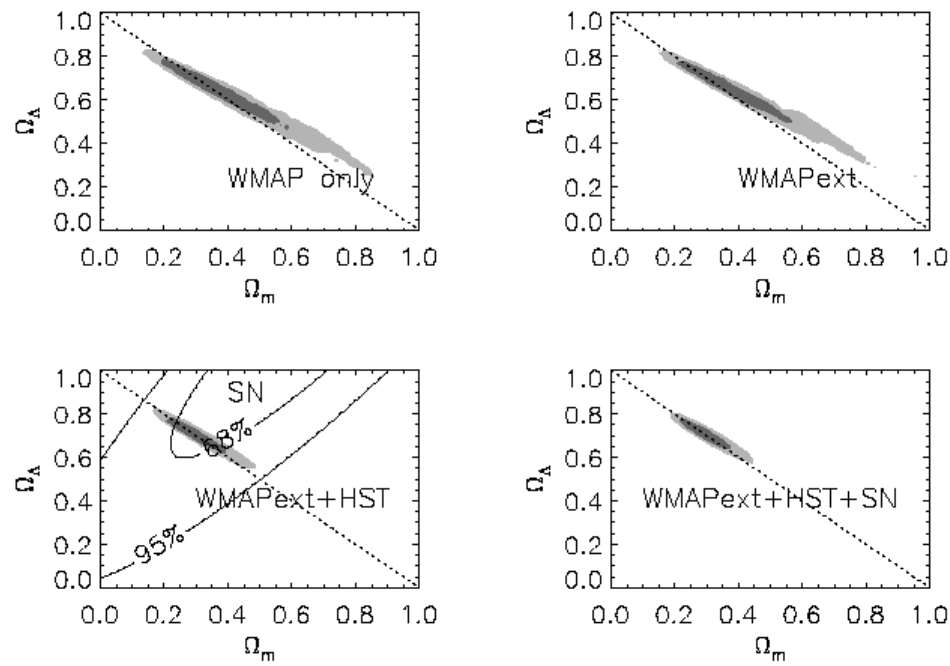

Figure 1: Current constraints on the relative abundance of Dark Matter and Dark Energy, as inferred using properties of the CMBR and measurements of large-scale structure. The diagonal line corresponds to a universe having total density, $\rho=\rho_{c}$, as discussed in the text [4].

determination obtained using CMBR and large-scale structure measurements.) This indicates the need for a missing component — called 'Dark Energy' — satisfying

$$
\Omega_{\Lambda}=\left(\frac{\rho_{D E}}{\rho}\right)_{\text {now }} \approx 70 \%
$$

Second, detailed tests of the Hubble expansion rate using supernovae show that the overall expansion rate of the universe, $H=\dot{a} / a$, appears to be increasing at present. As eq. (1.6) shows, this can only happen for positive energy density, $\rho>0$, if the total pressure is sufficiently negative, $p<-\frac{1}{3} \rho$. Since this is not true for any of the fluid components entertained to this point, something else must exist whose pressure is negative and at present dominates that of the other forms of matter.

Indeed, present-day understanding of the microscopic laws of Nature do allow pressure to be negative, and the simplest candidate is the vacuum itself for which Lorentz invariance implies its stress energy must satisfy $\left\langle T_{\mu \nu}\right\rangle \propto g_{\mu \nu}$, and so is predicted to have the equation of state

$$
p_{D E} \approx-\rho_{D E}
$$

This equation of state is assumed in what follows for Dark Energy, and agrees with the present observational bounds, which imply $p_{D E} / \rho_{D E}<-0.8$. Crucially, the amount of matter having this equation of state which reproduces the observed acceleration in the universal expansion is consistent with the energy density required to ensure $\sum_{i} \Omega_{i}=1$, as required by measurements of $H_{0}$ and $\kappa / a_{0}^{2}$. 


\subsection{Domination by radiation, matter and Dark Energy}

The different equations of state satisfied by radiation, non-relativistic matter (i.e. baryons and Dark Matter) and Dark Energy implies that their relative abundances differed in the past universe because their energy densities vary differently as the universe expands.

\section{Dependence of $\rho$ on $a$}

Notice that each of the above equations of state implies that the ratio $w_{i}=p_{i} / \rho_{i}$ is timeindependent, with

$$
w_{\mathrm{rad}}=\frac{1}{3}, \quad w_{\mathrm{m}}=0 \quad \text { and } \quad w_{D E}=-1
$$

and using this allows eq. (1.7) to be integrated to give

$$
\rho_{i}=\rho_{i 0}\left(\frac{a_{0}}{a}\right)^{\alpha_{i}}
$$

where $\alpha_{i}=3\left(1+w_{i}\right)$, and so

$$
\alpha_{\mathrm{rad}}=4, \quad \alpha_{\mathrm{m}}=3 \quad \text { and } \quad \alpha_{D E}=0
$$

Combining these results shows how the total energy density evolves with time given an initial density, $\rho_{0}$, which is divided into an initial fraction, $f_{i}=\rho_{i 0} / \rho_{0}$, of radiation (rad), non-relativistic matter $(m)$ and Dark Energy $(D E)$ :

$$
\rho(a)=\rho_{0}\left[f_{D E}+f_{m}\left(\frac{a_{0}}{a}\right)^{3}+f_{\mathrm{rad}}\left(\frac{a_{0}}{a}\right)^{4}\right]
$$

Because each term in the sum varies so differently with time, the history of the universe breaks up into epochs during each of which one term or another dominates, and so controls the overall change of $\rho(a)$, as shown in Fig. (2).

Exercise 3: Given the present-day abundances of radiation and matter, and using the relation $a_{0} / a=1+z$ (see Problem 2) between redshift and scale factor, show that the epoch where the energy density in radiation equals that of non-relativistic matter occurs at redshift $z_{\mathrm{eq}} \approx 3600$. Show that if Dark Matter did not exist (so baryons were the only non-relativistic matter), then the epoch of radiation-matter equality would have instead occurred much later, at $z_{\mathrm{Beq}} \approx 480$.

Notice in particular that the contribution to the Friedmann equation, eq. (1.5), of the "curvature term', $\kappa / a^{2}$, falls more quickly than does $\rho_{D E}$ (which does not fall at all), but more slowly than $\rho_{\text {rad }}$ and $\rho_{m}$. Since present-day measurements are consistent with $\kappa / a^{2} \approx 0$, it follows that curvature becomes less and less important the further back into the past we look. 


\section{Energy Density vs Scale Factor}

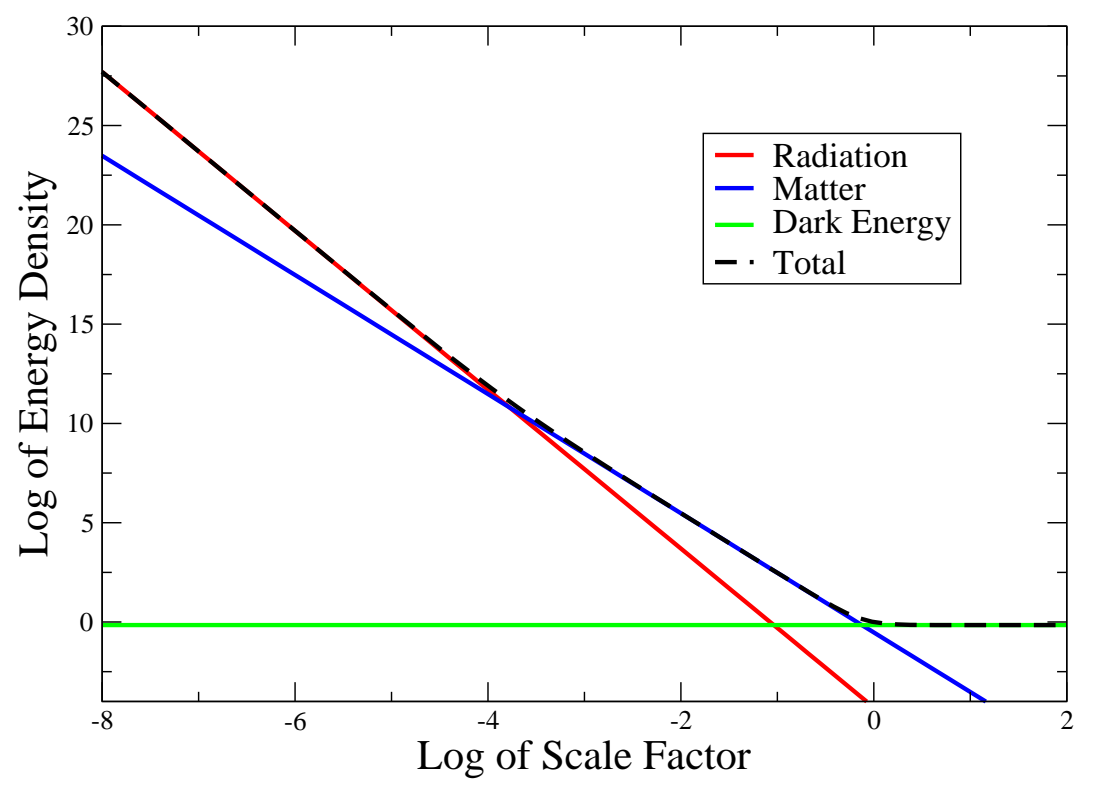

Figure 2: The energy density of radiation, non-relativistic matter and Dark Energy as a function of the universal scale factor, in units for which $\rho=a=1$ at present.

\section{Dependence of $a$ on $t$}

The dependence of $a$ on $t$ (and so also of $H$ on $a$ ), may be obtained from the Friedmann equation, eq. (1.5), using the above expression for $\rho(a)$. Setting $\kappa=0$, this implies

$$
\left(t-t_{0}\right)=\sqrt{3} M_{p} \int_{a_{0}}^{a} \frac{\mathrm{d} \hat{a}}{\hat{a} \sqrt{\rho(\hat{a})}},
$$

In general the right-hand side involves elliptic integrals, however it takes a simple form whenever $\rho(a)$ is dominated by one component of the cosmic fluid (as it almost always is). In this instance we have $\rho(a) \approx \rho_{0}\left(a_{0} / a\right)^{\alpha}$, where $\alpha=3(1+w)$, and so eq. (1.24) is easily integrated, leading to

$$
a(t)=a_{0}\left(\frac{t}{t_{0}}\right)^{\beta} \quad \text { and so } \quad H^{-1}(t)=\frac{t}{\beta},
$$

where $\beta=2 / \alpha=\frac{2}{3}(1+w)^{-1}$ for $w \neq-1$, and so $\beta=\frac{2}{3}$ when $w=0$ and $\beta=\frac{1}{2}$ when $w=\frac{1}{3}$. For later purposes, two things are worth remarking here. First, notice that $a(t)$ grows so quickly that it could have grown from zero size over a finite time interval. Second, $a(t)$ grows more slowly than does the Hubble length, $H^{-1}(t)$, so long as $\beta<1$ (i.e. for $w>-\frac{1}{3}$ ). This is true in particular for both radiation- and matter-dominated universes. 
For the exceptional case $w=-1$ we have $\alpha=0$ and so $\rho=\rho_{\star}$ is constant, so integration gives instead

$$
a(t)=a_{0} \exp \left[H_{\star}\left(t-t_{0}\right)\right] \quad \text { with } \quad H^{-1}(t)=H_{\star}^{-1}=\left(\frac{3 M_{p}^{2}}{\rho_{\star}}\right)^{1 / 2} .
$$

Here $a(t)$ grows more quickly than $H^{-1}(t)$ (which in this case does not grow at all).

\subsection{Major Events}

The Hot Big Bang model for cosmology assumes the universe was initially a hot soup of elementary particles, whose temperature was once at least 10 billion degrees. In broad brush strokes, its later evolution describes the cooling of this hot soup as the universe expands, for which conservation of entropy implies (for relativistic particles)

$$
T(t)=T_{0}\left(\frac{a_{0}}{a(t)}\right)
$$

For the purposes of later observations, there are two main consequences of such a cooling:

- Reduced Reaction Rates: Reaction rates in dilute systems are generically proportional to the number of participants per unit volume, because the reactants must be able to find one another before they are able to react. But since these particle densities fall as the universal volume grows, reaction rates also fall. This implies that one of the main trends of cosmology is the falling out of equilibrium of various thermal and chemical reactions.

- Formation of Bound States: A corollary of the previous point is the appearance of bound states of particles as the universe ages. Although the reactions forming bound states can always occur, at the earliest epochs temperatures are high enough to ensure that collisions very efficiently destroy these bound states - leaving very few to survive in equilibrium conditions. But inter-particle collisions become less violent as the temperature falls, so that eventually the reactions of formation can dominate to leave a population of primordial relict bound states.

At very early epochs phase transitions are also expected to play an important role in the cosmic evolution, but as yet there is no direct evidence that such transitions took place.

Most of the observational consequences of the Hot Big Bang revolve about the detection of such relics, together with the detailed measuring of their properties. A compressed history of the Hot Big Bang era then becomes a summary of which relics have been observed, and when they formed.

Big Bang Nucleosynthesis is the earliest cosmic event - occurring at a redshift $z_{B B N} \sim 10^{10}$ - for which we have direct observational evidence. At this time the temperature first cooled below about $1 \mathrm{MeV}$, at which point light nuclei (isotopes of Hydrogen, Helium, Lithium and Beryllium) first began to accumulate from their constituent protons and neutrons. Observational evidence for this

epoch comes from measuring the relative abundance of these primordial elements, and comparing 
the results with the predictions of nuclear physics. The success of these comparisons also provides a direct measure of the total baryon number density at this epoch, because this density strongly affects the various nuclear reaction rates.

Radiation - Matter Crossover is defined as the epoch when relativistic particles (radiation) stop being the dominant contribution to the cosmic energy density, passing this baton to non-relativistic Dark Matter (and baryons). As seen in Problem 3, this occurs at redshift $z_{\text {eq }} \sim 3600$. An important consequence of this crossover is in the speed with which gravity can enhance the growth of any initial density inhomogeneities. These can grow proportional to $a$ during matter domination, while they only grow logarithmically with $a$ during radiation domination.

Recombination is the epoch where free nuclei and electrons first combine into neutral atoms, at which point the universe first becomes transparent to photons having visible and near-UV wavelengths. For Hydrogen, which dominates the cosmic baryon abundance, this occurs over a comparatively short epoch (spread over a redshift interval of a few hundred) around $z_{\text {rec }} \sim 1100$. The CMBR has its origin as the light which is liberated by the universe's newfound transparency at this epoch, and so measurements of its temperature fluctuations, $\delta T / T \sim 10^{-5}$, provide direct information about the size of primordial density fluctuations in the cosmic environment at this time.

Galaxy Formation occurs once primordial density fluctuations have been amplified to the point that their evolution is no longer well-described as linear perturbations. This picture describes well the observed distribution of galaxies in the universe, but only given the presence of non-relativistic Dark Matter. Dark Matter is required since the amplitude of density fluctuations is known to be very small at the epoch of recombination, and does not grow strongly until after radiation-matter crossover (which occurs much later in the absence of Dark Matter).

\subsection{Special Initial Conditions}

In a nutshell, the previous section describes a simple and consistent picture of the relatively recent universe, described by Hot Big Bang cosmology, which is able to account for the many observations of the overall structure and evolution of the universe which are now being made. This success comes with some cost, however. Besides having to postulate the existence of two new forms of matter - Dark Matter and Dark Energy - for which we have no other evidence outside of cosmology - it is also necessary to start the universe off with a special kind of initial conditions. This section describes these initial conditions, together with a theoretical framework for their explanation in terms of the still-earlier history of the nascent universe.

It is common to couch the discussion of the special initial conditions required by the Big Bang model in terms of initial-condition 'problems', of which there are at least three types.

\section{The Flatness Problem}

The first problem concerns the spatial flatness of the present-day universe, which is suggested by observations of the temperature fluctuations in the CMBR. These observations indicate that the quantity $\kappa / a^{2}$ of the Friedmann equation, eq. (1.5), is at present consistent with zero. In order to 
see why this constitutes a problematic initial condition it is useful to divide this equation by $H(t)$ to give

$$
1+\frac{\kappa}{(a H)^{2}}=\frac{8 \pi G \rho}{3 H^{2}} \equiv \Omega(a) .
$$

Since the product $a H$ decreases with time (during both matter and radiation domination), this shows that the curvature term becomes more and more important as time passes.

The problem arises because observations indicate that at present $\Omega=\Omega_{0}$ is unity to within about $10 \%$. But during the matter-dominated era which is just ending the product $(a H)^{2} \propto a^{-1}$ so, using the result of Problem 3, at the point of radiation-matter equality we must have had

$$
\Omega\left(z_{\mathrm{eq}}\right)-1=\left(\Omega_{0}-1\right)\left(1+z_{\mathrm{eq}}\right)^{-1}=\frac{0.1}{3600} \approx 2.8 \times 10^{-5} .
$$

So if $\Omega_{0}$ is now within $10 \%$ of unity, then it was within a few tens of a millionth at the time of radiation-matter equality.

Earlier than this the universe was radiation-dominated, and so $(a H)^{2} \propto a^{-2}$. Since $z_{\mathrm{BBN}} \sim 10^{10}$ at the epoch of Big Bang Nucleosynthesis we have

$$
\Omega\left(z_{\mathrm{BBN}}\right)-1=\left[\Omega\left(z_{\mathrm{eq}}\right)-1\right]\left(\frac{1+z_{\mathrm{eq}}}{1+z_{\mathrm{BBN}}}\right)^{2}=\frac{0.1}{3600}\left(\frac{3600}{10^{10}}\right)^{2} \approx 3.6 \times 10^{-18},
$$

requiring $\Omega$ to be unity with an accuracy of roughly a part in $10^{18}$. The further back one goes, the more fantastic the accuracy with which we must start $\Omega$ near 1 in order to properly describe the universe as we now see it. One's discomfort of having the success of a theory hinge so sensitively on the precise value of an initial condition in this way is known as the Big Bang's Flatness Problem.

\section{The Horizon Problem}

The Big Bang's Horizon Problem asks why the initial universe is so very homogeneous. In particular, the temperature fluctuations of the CMBR only arise at the level of 1 part in $10^{5}$, and the question is why this temperature should be so incredibly uniform across the sky. Why is this regarded as a problem? After all, gasses on earth often have a uniform temperature, and this is usually understood as a consequence of thermal equilibrium because an initially inhomogeneous temperature distribution equilibrates by having heat flow between the hot and cold areas, until the gas is eventually all at the same temperature.

What makes it odd to see the same temperature in all directions of the sky in the Hot Big Bang model is that the universe generically expands so quickly - c.f. eq. (1.25) - that there has not been enough time for light to travel across the entire sky to bring the news as to what the common temperature is supposed to be. For instance, in a radiation-dominated universe $a(t)=a_{0}\left(t / t_{0}\right)^{1 / 2}$ and $H(t)=1 /(2 t)$ so the maximum proper distance that a light signal can travel by the time of recombination, $t_{\mathrm{rec}}$, is

$$
L_{\mathrm{rec}}=a_{\mathrm{rec}} \int_{0}^{t_{\mathrm{rec}}} \frac{\mathrm{d} \hat{t}}{a(\hat{t})}=2 t_{\mathrm{rec}}=\frac{1}{H_{\mathrm{rec}}}=\frac{1}{H_{0}}\left(\frac{a_{\mathrm{rec}}}{a_{0}}\right)^{3 / 2} \simeq \frac{1}{H_{0}}\left(\frac{1}{1100}\right)^{3 / 2},
$$

which uses $H \propto a^{-3 / 2}$ during matter domination (as is appropriate between recombination and now), and $a_{0} / a_{\text {rec }}=1+z_{\text {rec }} \simeq 1100$. 
Evaluating this using $H_{0}=75 \mathrm{~km} / \mathrm{sec} / \mathrm{Mpc}$ - or (keeping in mind our units for which $c=1$ ), $H_{0}^{-1} \simeq 13 \mathrm{Gyr} \simeq 4 \mathrm{Gpc}-$ gives $L_{\mathrm{rec}} \simeq 0.2 \mathrm{Mpc}$. Now the surface of last scattering for the CMBR at present is at a distance of order

$$
D_{0}=a_{0} \int_{t_{\mathrm{rec}}}^{t_{0}} \frac{\mathrm{d} \hat{t}}{a(\hat{t})}=3 t_{0}-3 t_{0}^{2 / 3} t_{\mathrm{rec}}^{1 / 3}=\frac{2}{H_{0}}\left[1-\left(\frac{a_{0}}{a_{\mathrm{rec}}}\right) \frac{H_{0}}{H_{\mathrm{rec}}}\right]=\frac{2}{H_{0}}\left[1-\left(\frac{a_{\mathrm{rec}}}{a_{0}}\right)^{1 / 2}\right],
$$

(using $a \propto t^{2 / 3}$ and $H \propto a^{-3 / 2}$ ) and so $D_{0} \simeq 2 / H_{0} \simeq 8 \mathrm{Gpc}$. But due to the intervening expansion of the universe, the angle subtended by $L_{\mathrm{rec}}$ placed at this distance away (in a spatially-flat geometry) is really $\theta \simeq L_{\mathrm{rec}} / D_{\text {rec }}$ where $D_{\mathrm{rec}}=\left(a_{\mathrm{rec}} / a_{0}\right) D_{0} \simeq 7 \mathrm{Mpc}$ is its distance at the time of last scattering, leading to $\theta \simeq 1^{\circ}$. Any two directions separated by more than this angle (about twice the angular size of the Moon, seen from Earth) are so far apart that light had not yet had time to reach one from the other since the universe's beginning. How could all the directions we see then have known they were all to equilibrate to the same temperature? It is very much as if we were to find a very uniform temperature distribution, immediately after the explosion of a very powerful bomb.

\section{A Defect Problem?}

A third problem called the Defect Problem ${ }^{1}$ can arise if one extrapolates the Big Bang back to times much earlier than the epoch of Big Bang Nucleosynthesis. Unlike the previous two problems, whether this problem really arises or not depends on the kind of physics describing these very short distances and high energies.

The potential problem arises if the physics of these scales implies the universe passed through the kind of phase transition during an earlier epoch, which produces topological defects. These defects can take the form of very massive particles (possibly carrying magnetic charges, and so called magnetic monopoles); long thin cosmic strings, which could now be stretched across the visible universe; or two-dimensional domain walls or sheets which cross the universe.

These kinds of objects can be fatal to successful late-time cosmology, depending on how many of them survive down to the present epoch. For instance if the defects are monopoles, then they typically are extremely massive and so behave like non-relativistic matter. But these can cause problems, depending on how abundantly they are produced - typically as much one per Hubble volume: $n \sim H^{3}$. For instance, since the energy density of such particles falls more slowly than does radiation as the universe expands, it can easily come to dominate the universe well before the nucleosynthesis epoch. This could cause the universe to expand (and so cool) too quickly as nuclei were forming, and so give the wrong abundances of light nuclei. Even if not sufficiently abundant during BBN, the energy density in relict defects can be inconsistent with measures of the current energy density.

This is clearly a much more hypothetical problem than are the other two, unless you are committed to a particular theory for the high-energy physics of the very early universe which produces these types of defects.

\footnotetext{
${ }^{1}$ Sometimes also known as the Monopole Problem.
} 


\section{Cosmic Inflation}

Cosmic Inflation was initially motivated as a way to understand how these special initial conditions of the Hot Big Bang model might be understood as naturally arising from the dynamics of a much earlier epoch. Quite compellingly, it has been found more recently also to provide a simple explanation for the origin of the primordial density fluctuations whose presence seeds both the observed temperature fluctuations of the CMBR and the formation of galaxies through gravitational collapse. (For textbook treatments of inflation, see ref. [6, 7, 3], and for recent reviews see ref. [8].)

\subsection{The Inflationary Paradigm}

The idea of Cosmic Inflation is that all three of the above problems can be solved if the history of the universe were to have undergone a period of accelerated expansion at some point in its very distant past. For example, suppose the universe were to temporarily pass through an epoch during which the dominant component of the cosmic fluid were to have an approximately constant energy density, $\rho=M_{I}^{4}$, which would require the equation of state $p=-\rho$. This is the equation of state used above for the vacuum, but now the value of the energy density is to be chosen to be much larger, such as $M_{I} \sim 10^{15} \mathrm{GeV}$.

During any such an epoch we have seen that the Hubble scale remains constant, $H_{I} \sim M_{I}^{2} / M_{p}$, and the scale factor grows exponentially, or inflates, according to eq. (1.26): $a(t)=a_{0} \exp \left[H_{I}(t-\right.$ $\left.t_{0}\right)$ ]. This expansion law implies that the combination $a H$ now grows exponentially with time, rather than falling as it did for matter- or radiation-domination. This last observation shows why this kind of expansion can solve the flatness, horizon and defect problems, as we now see.

Flatness Problem: Since $a H$ grows exponentially it does not take long for any initial curvature, $\kappa /(a H)$, to be diluted to extremely small values. Precisely how much dilution is required? For example, suppose the universe were radiation dominated all the way back to an extremely high temperature like $T_{M} \sim M_{I} \sim 10^{15} \mathrm{GeV}$. Since $T \propto 1 / a-$ and since light nuclei form at roughly $T_{B B N} \sim 1 \mathrm{MeV}$ - the universe expands by a factor $a_{B B N} / a_{M}=T_{M} / T_{B B N} \sim 10^{18}$ while cooling from $T_{M}$ to nucleosynthesis. Since $a H \propto 1 / a$ (radiation domination) during this time it also follows that $(a H)_{M} /(a H)_{B B N} \sim 10^{18}$. Comparing with eq. (1.30) shows that the universe must have been very flat indeed at this early epoch:

$$
\begin{aligned}
\Omega\left(z_{M}\right)-1 & \sim\left(\Omega_{0}\left(z_{\mathrm{BBN}}\right)-1\right)\left[\frac{(a H)_{\mathrm{BBN}}}{(a H)_{M}}\right]^{2} \\
& \sim 3.6 \times 10^{-54}\left(\frac{10^{15} \mathrm{GeV}}{T_{M}}\right)^{2} .
\end{aligned}
$$

Since $(a H)_{t} /(a H)_{0}=a(t) / a_{0}=\exp \left[H_{I}\left(t-t_{0}\right)\right]$ during exponential expansion, even such a small initial condition would very easily be explained if the radiation-dominated epoch were preceded by exponential expansion for a period of time, $\Delta t$, satisfying

$$
N_{e} \equiv H_{I} \Delta t \gtrsim \frac{1}{2} \ln \left(3 \times 10^{53}\right) \simeq 62 \text {. }
$$


That is, under these circumstances generic initial conditions get sucked towards very flat geometries by inflation, with sufficient flatness arising even in extreme circumstances given about $60 e$-foldings of inflation.

Horizon Problem: This type of accelerated expansion can also solve the horizon problem because once $a H$ is increasing physical distance scales, $L(t)=a(t) \ell$, grow more quickly than does the Hubble length, $H^{-1}(t)$. Modes which were initially shorter than the Hubble length eventually can be stretched to be larger than the Hubble scale. The larger the co-moving scale, $\ell$, that is involved, the earlier it grows larger than the Hubble length during inflation. This makes it possible to have ordinary causal processes be stretched during inflationary times to appear at late times as if they were too far apart to be causally related.

How much inflation is required to make this work? The largest proper scales presently visible to us are of order $H_{0}^{-1} \sim 4 \mathrm{Gpc}$, and so we focus our attention to scales that are presently this size, $L\left(t_{0}\right)=a_{0} \ell \sim H_{0}^{-1}$, or $\ell \sim 1 /(a H)_{0}$. Because $a H$ decreases during radiation- and matter-dominated epochs, such scales satisfied $L(t)>H^{-1}(t)$ at earlier times, with for example

$$
\frac{L\left(t_{B B N}\right)}{H_{B B N}^{-1}}=\ell(a H)_{B B N}=\left[\frac{(a H)_{B B N}}{(a H)_{\mathrm{eq}}}\right]\left[\frac{(a H)_{\mathrm{eq}}}{(a H)_{0}}\right]=\left(\frac{a_{\mathrm{eq}}}{a_{B B N}}\right)\left(\frac{a_{0}}{a_{\mathrm{eq}}}\right)^{1 / 2} \simeq 2 \times 10^{8},
$$

at the epoch of nucleosynthesis.

During exponential expansion, however, $L / H^{-1}$ grows and so we ask how much exponential expansion is required in order to ensure that this scale also satisfies $L<H^{-1}$ at some earlier time, $t_{\text {he }}$, called the time of horizon exit. For times earlier than this (during or before inflation) causal processes can be at work to explain things like the present-day uniformity of the CMB temperature over these scales. (See Figure 5 for a sketch of the relative sizes of $L$ and $H^{-1}$, during and after inflation.)

For simplicity we assume that inflation ends when $t=t_{\mathrm{end}}$ and the universe then makes an immediate transition from an inflationary epoch, where $\rho=\rho_{I}=M_{I}^{4}$ is approximately constant, to a radiation-dominated epoch whose initial reheat temperature is also $T \sim M_{I}$ (i.e. reheats with perfect efficiency). In this case at the epoch of horizon exit we have (by assumption) $L\left(t_{\mathrm{he}}\right)=\ell a_{\mathrm{he}}=H_{\mathrm{he}}^{-1}$ and so $\ell=(a H)_{0}^{-1}=(a H)_{\mathrm{he}}^{-1}$. Consequently,

$$
1=\frac{a_{0} H_{0}}{a_{\mathrm{he}} H_{\mathrm{he}}}=\left(\frac{a_{\mathrm{end}} H_{\mathrm{end}}}{a_{\mathrm{he}} H_{\mathrm{he}}}\right)\left(\frac{a_{\mathrm{eq}} H_{\mathrm{eq}}}{a_{\mathrm{end}} H_{\mathrm{end}}}\right)\left(\frac{a_{0} H_{0}}{a_{\mathrm{eq}} H_{\mathrm{eq}}}\right),
$$

which we solve for $a_{\mathrm{end}} / a_{\mathrm{he}}=e^{N_{e}}=e^{H_{I}\left(t_{\mathrm{end}}-t_{\mathrm{he}}\right)}$, assuming a constant energy density during inflation, and so $H_{\mathrm{he}} \approx H_{\mathrm{end}}$. Using, as above, $\left(a_{\mathrm{eq}} H_{\mathrm{eq}}\right) /\left(a_{0} H_{0}\right)=\left(a_{0} / a_{\mathrm{eq}}\right)^{1 / 2} \simeq 60$, and $\left(a_{\mathrm{eq}} H_{\mathrm{eq}}\right) /\left(a_{\mathrm{end}} H_{\mathrm{end}}\right)=$ $a_{\mathrm{end}} / a_{\mathrm{eq}}=T_{\mathrm{eq}} / T_{M}$ with $T_{\mathrm{eq}} \sim 3 \mathrm{eV}$ leads to

$$
N_{e} \sim \ln \left[\left(3 \times 10^{23}\right) \times 60\right]+\ln \left(\frac{T_{M}}{10^{15} \mathrm{GeV}}\right) \approx 58+\ln \left(\frac{T_{M}}{10^{15} \mathrm{GeV}}\right) .
$$

Again we see that roughly $60 e$-foldings of exponential expansion can provide a framework for explaining how causal physics might provide the observed correlations that are observed in the CMBR over the largest scales. We shall see below that life is even better than this, because in 
addition to providing a framework in which a causal understanding of correlations could be solved, inflation itself can provide the mechanism for explaining these correlations (given an inflationary scale of the right size).

Defect Problem: Inflation can also solve the defect problem — within theories for which this needs solving - for similar reasons. Consider for example monopoles, which are typically predicted to be produced one per Hubble volume, $H_{f}^{-3}$, at the epoch where they are formed. Consequently their number density at that time would be $n_{f} \sim H_{f}^{3}$. The number density at later times is therefore $n=H_{f}^{3}\left(a_{f} / a\right)^{3}$ and so the number of defects per Hubble volume at later times is $N_{\text {def }}=n H^{-3}=$ $\left[(a H)_{f} /(a H)\right]^{3}$. As such it is clear that this number gets enormously diluted if the monopoles are produced before inflation, because of the enormous exponential suppression which is then possible for $(a H)_{f} /(a H)$.

\subsection{Single-Field Models}

So far so good, but the devil is in the details. Obtaining the benefits of such an exponential expansion requires two things: $(i)$ some sort of physics which can hang the universe up for a relatively long period with a vacuum-dominated equation of state, $p \approx-\rho$; and (ii) some mechanism for ending this epoch to allow the later appearance of the radiation-dominated epoch within which the usual Big Bang cosmology starts. Although a number of models exist for the kinds of physics which might do this, none of these models yet seems completely compelling. This section describes some of the very simplest such models, in order to see some of their successes and limitations, and to see what their implications can be for the large-scale structure seen in the later universe.

No way is known to obtain inflation simply using the known particles and interactions, and so inflationary models are characterized by what kind of new physics is invented to describe the inflationary dynamics. For the vast majority of models this new physics comes from the dynamics of a scalar field, $\varphi(x)$, (called the inflaton) which can be thought to be an order parameter characterizing the nature of the vacuum in the theory which describes the very high energy physics relevant to inflationary cosmology. Although the field $\varphi$ can in principle depend on both position and time, inflation turns out rapidly to smooth out spatial variations, and so it suffices to study $\varphi=\varphi(t)$.

The simplest such a relativistic order parameter has a dynamics which is determined by a potential energy, $V(\varphi)$, and satisfies the following field equation,

$$
\ddot{\varphi}+3 H \dot{\varphi}+V^{\prime}=0
$$

where $V^{\prime}=\mathrm{d} V / \mathrm{d} \varphi$. Its gravitational influence is described by the usual Friedmann and acceleration equations, but including also a $\varphi$-dependent contribution to the energy and pressure: $\rho=\rho_{\text {rad }}+$ $\rho_{\mathrm{m}}+\rho_{\varphi}$ and $p=\frac{1}{3} \rho_{\mathrm{rad}}+p_{\varphi}$, where $\rho_{\text {rad }}$ and $\rho_{\mathrm{m}}$ describe the energy density of relativistic and non-relativistic matter, and

$$
\rho_{\varphi}=\frac{1}{2} \dot{\varphi}^{2}+V(\varphi) \quad \text { and } \quad p_{\varphi}=\frac{1}{2} \dot{\varphi}^{2}-V(\varphi) .
$$

We imagine the Dark Energy of the modern epoch to correspond to there being a very small constant term in $V$, which is assumed to presently dominate. 
As is easy to check, with these choices energy conservation for the $\varphi$ field $-\dot{\rho}_{\varphi}+3(\dot{a} / a)\left(\rho_{\varphi}+\right.$ $\left.p_{\varphi}\right)=0$ follows from the field equation, eq. (2.6), and so $\varphi$ exchanges energy with the rest of the cosmic ingredients purely through their mutual gravitational interactions. The $\varphi$ field is not imagined to be in thermal equilibrium with itself or with the other kinds of matter, and this is selfconsistent because it couples to the other matter only gravitationally (which is too weak to establish equilibrium).

\section{Slow-Roll Inflation}

We seek a solution to these equations for $\varphi(t)$ for which the Hubble parameter, $H$, is approximately constant. This is ensured if the total energy density is dominated by $\rho_{\varphi}$, with $\rho_{\varphi}$ also approximately constant. Energy conservation then requires the pressure to satisfy $p_{\varphi} \approx-\rho_{\varphi}$. It does not matter here that $\varphi$ is not in equilibrium, since for $\varphi$ we ask that this relation between $\rho_{\varphi}$ and $p_{\varphi}$ to follow as a consequence of the field equations and not as an equation of state. Inspection of eqs. (2.7) shows that the regime of interest is when the $\varphi$ kinetic energy is negligible compared with its kinetic energy: $\frac{1}{2} \dot{\varphi}^{2} \ll V(\varphi)$ since then $p_{\varphi} \approx-V(\varphi) \approx-\rho_{\varphi}$. So long as $V(\varphi)$ is also much larger than any other energy densities, it would dominate and $H^{2} \approx V /\left(3 M_{p}^{2}\right)$ would then be approximately constant.

What properties must $V(\varphi)$ satisfy in order to allow such an extended period of slow rolling? Clearly the field equation (2.6) only permits precisely time-independent solutions, $\varphi=\varphi_{0}$, at points where the potential is stationary, $V^{\prime}\left(\varphi_{0}\right)=0$. As we now quantify, a sufficient condition for having a long period of time with $\varphi$ very slowly moving requires both $\dot{\varphi}$ and $\ddot{\varphi}$ to remain small for the entire inflationary period, and so requires both $V^{\prime}$ and $V^{\prime \prime}$ to be close to zero for a sufficiently broad range of $\varphi$.

More specifically, in order to have a prolonged slow roll we must demand $\ddot{\varphi} \ll H \dot{\varphi}$, which allows eq. (2.6) to be approximately written in the following slow-roll approximation

$$
\dot{\varphi} \approx-\left(\frac{V^{\prime}}{3 H}\right) \text {. }
$$

Using this in the condition $\frac{1}{2} \dot{\varphi}^{2} \ll V$ shows $V$ must satisfy $\left(V^{\prime}\right)^{2} /\left(9 H^{2} V\right) \ll 1$, or

$$
\varepsilon \equiv \frac{1}{2}\left(\frac{M_{p} V^{\prime}}{V}\right)^{2} \ll 1
$$

A self-consistency condition for using eq. (2.8) throughout inflation is the requirement that $\ddot{\varphi}$ remains small. Differentiating eq. (2.8) with respect to $t$, and using the approximate constancy of $H$ gives $\ddot{\varphi} \approx-V^{\prime \prime} \dot{\varphi} /(3 H)$. Demanding this remain small (in absolute value) compared with $3 H \dot{\varphi}$, then gives $\left|V^{\prime \prime} /(3 H)^{2}\right| \ll 1$, or $|\eta| \ll 1$ where

$$
\eta \equiv \frac{M_{p}^{2} V^{\prime \prime}}{V}
$$

As we shall see, all of the important predictions of single-field slow-roll inflation for density fluctuations can be expressed in terms of these two small parameters, $\varepsilon$ and $\eta$, together with the value of the Hubble parameter, $H$, during inflation. 
We have seen that the success of inflation relies on obtaining sufficient expansion, and so it is convenient to relate the amount of expansion directly to the distance $\varphi$ traverses in field space. To this end, rewriting eq. (2.8) in terms of $\varphi^{\prime} \equiv \mathrm{d} \varphi / \mathrm{d} a$, leads to

$$
\frac{\mathrm{d} \varphi}{\mathrm{d} a}=\frac{\dot{\varphi}}{\dot{a}}=-\frac{V^{\prime}}{3 a H^{2}}=-\frac{M_{p}^{2} V^{\prime}}{a V},
$$

which when integrated between the initial value, $\varphi_{i}$, and final value, $\varphi_{\text {end }}$, implies the universal expansion during inflation is given by $a_{\text {end }} / a_{i} \equiv \exp \left(N_{I}\right)$, with

$$
N_{I}\left(\varphi_{i}\right)=\int_{a_{i}}^{a_{\text {end }}} \frac{\mathrm{d} a}{a}=\int_{\varphi_{\text {end }}}^{\varphi_{i}} \mathrm{~d} \varphi\left(\frac{V}{M_{p}^{2} V^{\prime}}\right)=\frac{1}{M_{p}} \int_{\varphi_{\text {end }}}^{\varphi_{i}} \frac{\mathrm{d} \varphi}{\sqrt{2 \varepsilon}} .
$$

Since $\varphi_{\text {end }}$ can be defined by the point where the slow-roll parameters are no longer small, this last equation can be read as defining $\varphi_{i}\left(N_{I}\right)$, as a function of the desired number of $e$-foldings. This is most usefully applied to finding the number of $e$-foldings, $N_{e}$, between the the epoch of horizon exit - as defined below eq. (2.4) - and the end of inflation: $N_{e} \equiv N_{I}\left(\varphi_{\text {he }}\right)$, since it is this quantity which is constrained to be large by the horizon and flatness problems. Notice also that if $\varepsilon$ were approximately constant during inflation, then eq. (2.12) implies that $N_{I} \approx\left(\varphi_{i}-\varphi_{\text {end }}\right) /\left(\sqrt{2 \varepsilon} M_{p}\right)$. In such a case $\varphi$ must traverse a range larger than $O\left(M_{p}\right)$ between $\varphi_{i}$ and $\varphi_{\text {end }}$ in order to obtain 60 or more $e$-foldings, unless $\varepsilon \lesssim 10^{-4}$.

\section{Large- and Small-Field Examples}

Consider, for example, the special case where

$$
V=A+\frac{1}{2} B \varphi^{2}+\frac{1}{4} \lambda^{2} \varphi^{4}
$$

and so for which

$$
V^{\prime}=B \varphi+\lambda^{2} \varphi^{3} \quad \text { and } \quad V^{\prime \prime}=B+3 \lambda^{2} \varphi^{2} .
$$

There are two examples of slow rolls which arise in this case and which (for observational purposes) are representative of two of the main classes of inflationary models.

\section{Large-Field Inflation:}

For very large $\varphi$ we have $V \approx \frac{1}{4} \lambda^{2} \varphi^{4}, V^{\prime} \approx \lambda^{2} \varphi^{3}$ and $V^{\prime \prime} \approx 3 \lambda^{2} \varphi^{2}$ and so

$$
\varepsilon \approx \frac{1}{2}\left(\frac{4 M_{p}}{\varphi}\right)^{2} \quad \text { and } \quad \eta \approx \frac{12 M_{p}^{2}}{\varphi^{2}}
$$

while the scale for inflation is $M_{I}^{4} \equiv V \approx \frac{1}{4} \lambda^{2} \varphi^{4}$ and so $H_{I} \approx \lambda \varphi^{2} /\left(2 \sqrt{3} M_{p}\right)$. [More generally, for $M_{I}^{4}=V \approx \frac{1}{n} \lambda^{2} \varphi^{n}, V^{\prime} \approx \lambda^{2} \varphi^{n-1}$ and $V^{\prime \prime} \approx(n-1) \lambda^{2} \varphi^{n-2}$ and so $\varepsilon \approx \frac{1}{2}\left(n M_{p} / \varphi\right)^{2}$ and $\eta \approx$ $n(n-1) M_{p}^{2} / \varphi^{2}$, and the Hubble scale for inflation is $H_{I} \approx \lambda \varphi^{2} /\left(\sqrt{3 n} M_{p}\right)$.]

In this case $\eta \approx \frac{3}{2} \varepsilon>0$ and both are small provided $\varphi \gg M_{p}$ (which is consistent with the large- $\varphi$ approximation being used). In this regime $\varphi$ (and so also $V$ and $H$ ) remains approximately constant despite there being no stationary point for $V$ at large $\varphi$ because Hubble friction keeps $\varphi$ 
from sliding down the potential very quickly. Since $\varphi$ evolves towards smaller values, eventually slow roll ends once $\eta$ and $\varepsilon$ become $O(1)$. Since $\eta>\varepsilon$, it is convenient to define $\varphi_{\text {end }}$ by $\eta=\frac{3}{4}$, which implies $\varphi_{\text {end }}=4 M_{p}$.

The number of $e$-foldings between horizon exit and $\varphi_{\mathrm{end}}=4 M_{p}$ is given by eq. (2.12), which becomes

$$
N_{e} \equiv N_{I}\left(\varphi_{\mathrm{he}}\right)=\int_{\varphi_{\mathrm{end}}}^{\varphi_{\mathrm{he}}} \mathrm{d} \varphi\left(\frac{\varphi}{4 M_{p}^{2}}\right)=\frac{\varphi_{\mathrm{he}}^{2}}{8 M_{p}^{2}}-2 .
$$

This shows that obtaining $N_{e}>60 e$-foldings requires choosing $\varphi_{\text {he }} \gtrsim 22 M_{p}$.

\section{Small-Field Inflation:}

Alternatively, imagine again using the potential of eq. (2.13), but instead assuming $B=-\mu^{2}<0$ and so $V$ has a local maximum at $\varphi=0$. Sufficiently near this maximum,

$$
\varphi^{2} \ll \min \left(\frac{2 A}{\mu^{2}}, \frac{2 \mu^{2}}{\lambda^{2}}\right),
$$

we have $V \approx A \equiv M_{I}^{4}, V^{\prime} \approx-\mu^{2} \varphi$ and $V^{\prime \prime} \approx-\mu^{2}$. If so, the slow-roll parameters become

$$
\varepsilon \approx \frac{1}{2}\left(\frac{\mu^{2} M_{p} \varphi}{A}\right)^{2} \quad \text { and } \quad \eta \approx-\left(\frac{\mu^{2} M_{p}^{2}}{A}\right) .
$$

In this case $\eta<0$ and $\varepsilon=\frac{1}{2}\left(\eta \varphi / M_{p}\right)^{2} .|\eta|$ is small provided $\mu^{2} M_{p}^{2} \ll A$ and since we have assumed $\varphi$ to be small we see that generically in this case $\varepsilon \ll|\eta|$. Again the slow-roll regime is consistent with the small- $\varphi$ approximation with which we start. The inflationary scale is $V \approx A=$ $M_{I}^{4}$, and so $H=M_{I}^{2} /\left(\sqrt{3} M_{p}\right)$.

Physically, the scalar potential in this case can dominate the energy density because there is always an unstable solution to the equations of motion corresponding to sitting with $\varphi$ precisely at rest at the local maximum, where $V^{\prime}=0$. Solutions near this static solution can therefore be very slow if they start sufficiently close to the maximum, or if the maximum is sufficiently shallow. As we see below, only the second of these two options provides a bona fide inflationary model.

Since $\eta$ is constant, the end of inflation occurs once either $\varepsilon$ becomes $O(1)$ or once the small$\varphi$ conditions, eq. (2.17), break down. Since $\varepsilon=O(1)$ requires $\varphi=O\left(M_{p} /|\eta|\right)$, it is well outside of the assumed small-field regime and so it is the failure of eq. (2.17) which kicks in first: $\varphi_{\text {end }}^{2} \sim$ $\min \left(2 A / \mu^{2}, 2 \mu^{2} / \lambda^{2}\right)$. The total number of $e$-foldings after $\varphi=\varphi_{\text {he }}$, becomes in this case

$$
N_{e} \equiv N_{I}\left(\varphi_{\mathrm{he}}\right)=\int_{\varphi_{\mathrm{end}}}^{\varphi_{\mathrm{he}}} \mathrm{d} \varphi\left(\frac{A}{M_{p}^{2} B \varphi}\right)=\frac{A}{M_{p}^{2} \mu^{2}} \ln \left(\frac{\varphi_{\mathrm{end}}}{\varphi_{\mathrm{he}}}\right)=\frac{1}{|\eta|} \ln \left(\frac{\varphi_{\mathrm{end}}}{\varphi_{\mathrm{he}}}\right) .
$$

Since this only depends logarithmically on $\varphi_{\text {end }} / \varphi_{\text {he }}$, obtaining $N_{e} \gtrsim 60$ generically requires $|\eta| \lesssim$ $1 / 60=0.017$. Taking, for instance, $A=M_{I}^{4}$ with $M_{I}=10^{14} \mathrm{GeV}$, then implies $\mu \lesssim M_{I}^{2} / M_{p}=10^{10}$ $\mathrm{GeV}$.

Another way to make $N_{e}$ large would be to take $\varphi_{\mathrm{he}} \rightarrow 0$, since in this limit $N_{e} \rightarrow \infty$ corresponding to the solution which sits at the top of the maximum for an indefinitely long period. 
At first sight this choice seems attractive because it appears always to be possible, regardless of how steeply the potential falls away from this maximum. However, in reality the inflaton field is subject to fluctuations, such as due to quantum vacuum fluctuations which arise because the scalarfield Hamiltonian - for which the vacuum is an eigenstate - does not commute with the field, $\phi$, itself. $\varphi$ is only a classical approximation to $\langle\phi\rangle$, but in an exponentially-expanding universe the fluctuations about this value turn out to be of order $\delta \varphi \sim H_{I}$. Generically, then, we can only choose $\varphi_{\text {he }}=0$ to within an accuracy $\delta \varphi \sim H_{I}$, and so should restrict $\varphi_{\text {he }} \gtrsim H_{I}$. For the potential of current interest this implies $\varphi_{\mathrm{he}} \gtrsim H_{I} \sim M_{I}^{2} / M_{p}$ and so since $\varphi_{\mathrm{end}} \sim \min \left(M_{I}^{2} / \mu, \mu / \lambda\right)$, we have $\varphi_{\text {end }} / \varphi_{\text {he }} \lesssim M_{p} / \mu$ or $\varphi_{\text {end }} / \varphi_{\text {he }} \lesssim \mu M_{p} /\left(\lambda M_{I}^{2}\right) \sim \sqrt{|\eta|} / \lambda$, showing that large values for $N_{e}$ really do require $|\eta|$ to be small.

\section{Consistency of the Approximations}

It is important for any inflationary model to ask whether the choices made for inflation are consistent with approximations which are made when writing down a scalar potential. There are three important criteria which must be satisfied.

1. Perturbation theory: Analyzing the dynamics of $\varphi$ as a classical field (rather than a quantum one) assumes the semi-classical approximation. For instance, the validity of this is justified in the case studied above when $\lambda \ll 1$ and $\varphi^{2} \lesssim|B| / \lambda^{2}$.

2. Quantum Gravity: Neglect of the complications of quantum gravity require that no energy densities should ever be allowed to be greater than Planck density. That is, $\frac{1}{2} \dot{\varphi}^{2} \ll M_{p}^{4}$ and $V \ll M_{p}^{4}$. In the example above this implies choosing $A \ll M_{p}^{4}, \varphi / M_{p} \ll M_{p} / \mu$ and $\varphi / M_{p} \ll \lambda^{-1 / 2}$. Note this can permit the large- $\varphi$ regime, $\varphi \gg M_{p}$, provided $\lambda$ and $\mu / M_{p}$ are sufficiently small.

3. High-Energy Corrections to $V$ : Typically the integrating out of higher-energy physics generates corrections to the shape of $V$, with the contributions due to physics at mass scale $M$ generically contributing terms of order $\delta V \sim \varphi^{k} / M^{k-4}$ for all possible choices for $k$. If the success of the inflationary model depends on the particular form for $V$ it is therefore necessary to understand why these corrections are not present or important in the example of interest. For small-field inflation it is the absence of terms with $k \leq 4$ which require explanation, since these are not automatically suppressed by powers of $\varphi / M$. Since $M$ is typically smaller than $M_{p}$, large-field inflation is sensitive to a potentially enormous range of $k$ 's, which is to say that it must be understood why these corrections do not change the large-field form of the potential.

\subsection{Primordial Density Fluctuations}

One of the successes of the Hot Big Bang is its description of the origins of galaxies, which are understood as the final result of the gravitational amplification of what were initially very small density inhomogeneities. This picture of structure formation very successfully describes the observed distribution of galaxies, as well as how this distribution correlates with the observed small 
temperature fluctuations of the CMBR. The structure-formation picture assumes the existence of initially small primordial fluctuations about the homogeneous universe, and its success depends on assumptions made about their detailed properties. These are simply taken as an initial condition of the Big Bang Model, with no attempt made to understand their origin.

Although originally motivated as a solution to the horizon and flatness problems, a bonus for inflationary models is their subsequent success in predicting the properties of the primordial fluctuations which the Hot Big Bang requires. This prediction describes the fluctuations as being due to ordinary microscopic quantum fluctuations of the inflaton field, $\delta \varphi$, and the metric, $\delta g_{\mu \nu}$, which become stretched up to cosmologically interesting scales by the inflationary expansion of the universe. This section provides a heuristic description of these fluctuations before quoting the final results which follow from more sophisticated calculations.

\section{Fluctuation Phenomenology}

Before describing what inflation can say about the properties of primordial fluctuations, first recall how these fluctuations are characterized. Since the universe seems to be spatially flat, it is convenient for these purposes to use $\kappa=0$ for the background geometry, and to Fourier transform fluctuating quantities. For instance, writing the fluctuating energy density in non-relativistic matter as $\rho(\mathbf{r}, t)=\rho_{\mathrm{m}}(t)[1+\delta(\mathbf{r}, t)]$, we have

$$
\delta(\mathbf{r}, t)=\int \frac{\mathrm{d}^{3} k}{(2 \pi)^{3}} \delta_{k}(t) \exp [i \mathbf{k} \cdot \mathbf{x}]=\int \frac{\mathrm{d}^{3} k}{(2 \pi)^{3}} \delta_{k}(t) \exp [i(\mathbf{k} / a) \cdot \mathbf{r}],
$$

where homogeneity and isotropy of the background cosmology implies $\delta_{k}(t)$ depends only on $k=|\mathbf{k}|$ and $t$. $\mathbf{x}$ here denotes the co-moving coordinate, corresponding to physical distance $\mathbf{r}=a \mathbf{x}$, so the physical wavelength associated with co-moving wave-number $k$ is $\lambda=2 \pi a / k$.

A useful statistic for quantifying the galaxy distribution is the density-density autocorrelation function, $\xi_{\rho}(\mathbf{r}, t)$, defined by

$$
\xi_{\rho}(\mathbf{r})=\left\langle\delta\left(\mathbf{r}^{\prime}+\mathbf{r}\right) \delta\left(\mathbf{r}^{\prime}\right)\right\rangle=\int \frac{\mathrm{d}^{3} k}{(2 \pi)^{3}} P_{\rho}(k) \exp [i(\mathbf{k} / a) \cdot \mathbf{r}],
$$

where the average is over all $\mathbf{r}^{\prime}$. This measures how likely it is to find a density excursion at a physical distance $\mathbf{r}$ from a given density excursion. The integrand in the second equality defines the power spectrum of density fluctuations, $P_{\rho}(k)$, which can be related to $\delta_{k}$ by $P_{\rho}(k) \propto\left|\delta_{k}\right|^{2}$. A dimensionless measure of the power spectrum is obtained by performing the angular integration in eq. (2.21), leading to

$$
\xi_{\rho}(\mathbf{r})=\int_{0}^{\infty} \frac{\mathrm{d} k}{k} \Delta_{\rho}^{2}(k) \frac{\sin (k r / a)}{k r / a}
$$

where $\Delta_{\rho}^{2}=k^{3} P_{\rho}(k) /\left(2 \pi^{2}\right)$.

Theoretically, the evolution of linear perturbations within the Hot Big Bang allows $P_{\rho}(k, t)$ to be computed in terms of the primordial spectrum, $P_{\rho}^{0}(k)$, of initial density distributions, according to

$$
P_{\rho}\left(k, t_{\text {now }}\right)=P_{\rho}^{0}(k) \mathscr{T}\left(k, t_{0}, t_{\text {now }}\right),
$$




\section{Linear Power Spectrum}

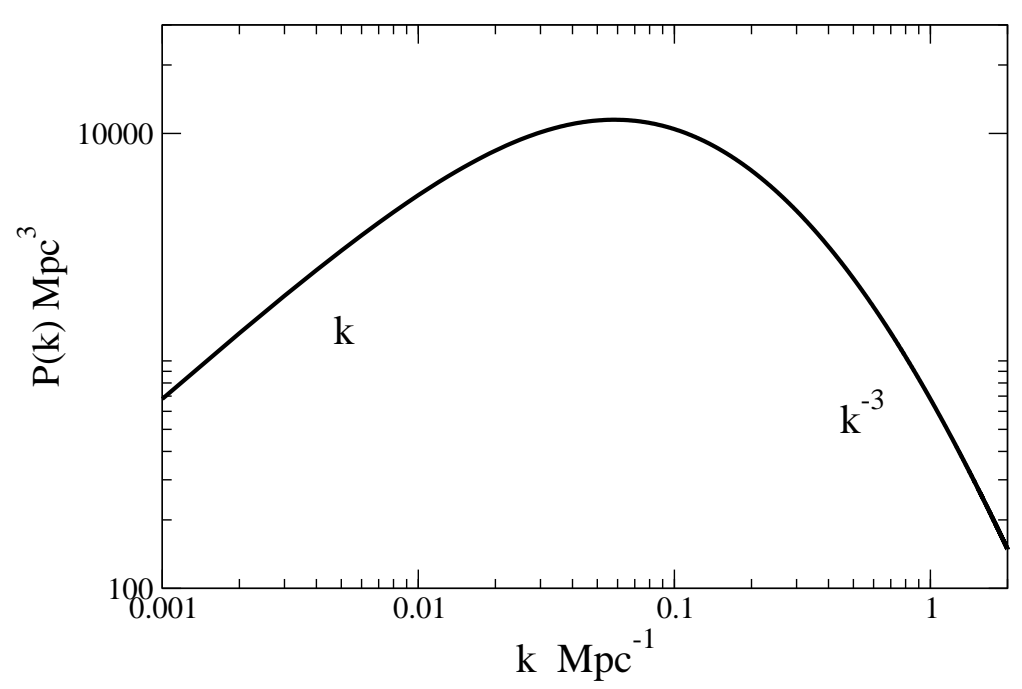

Figure 3: A sketch of the linear density power spectrum, $P_{\rho}(k)$.

where $\mathscr{T}\left(k, t, t^{\prime}\right)$ is a calculable transfer function. $\mathscr{T}$ has the property that it is approximately independent of $k$ for small $k$, and is proportional to $k^{-4}$ for large $k$, with the transition between these regimes occurring for co-moving wave-numbers satisfying $k \simeq(a H)_{\mathrm{eq}}$ at the epoch of radiationmatter equality.

Physically, this form for $\mathscr{T}$ arises because those modes satisfying $k>k_{\text {eq }} \equiv(a H)_{\text {eq }}$ re-enter the Hubble scale before radiation-matter equality, while those with $k<k_{\text {eq }}$ do so afterwards. However, density fluctuations only grow logarithmically with $a$ during radiation domination, but can grow proportional to $a$ during matter domination. All other things being equal, one therefore expects modes with $k<k_{\text {eq }}$ to have a $k$-dependent amplitude, because they grow over the $k$-dependent time interval during which the universe expands by a factor $a_{0} / a_{k} \propto k^{2}$, where $a_{k}$ is defined as the scale factor at the epoch where $a H=k$, and we use that $a H \propto a^{-1 / 2}$ during matter domination to conclude $a_{k} \propto k^{-2}$. By contrast, modes with $k>k_{\text {eq }}$ all start growing at radiation-matter equality, and so are amplified by a $k$-independent factor: $a_{0} / a_{\text {eq }}$. This implies modes with $k>k_{\text {eq }}$ are stunted by an amount proportional to $\left(k_{\mathrm{eq}} / k\right)^{2}$ relative to what one would get by extrapolating from the amplitude of modes with $k<k_{\text {eq }}$, so their contribution to the power spectrum is suppressed by $\mathscr{T} \propto 1 / k^{4}$.

Observationally, $P_{\rho}(k)$ can be related to the galaxy-galaxy correlation function, which can be measured from surveys of galaxy distributions. It can also be used to compute the temperature fluctuations observed in the CMBR. These measure the correlations between the temperature deviations seen in two directions, $\mathbf{n}$ and $\mathbf{n}^{\prime}$, as a function of their relative direction, $\cos \theta=\mathbf{n} \cdot \mathbf{n}^{\prime}$, with the result averaged over all possible orientations of these two vectors (for fixed relative direction, $\theta$ ) in the sky. The result is conventionally expressed by expanding in a Legendre series,

$$
\left\langle\frac{\delta T}{T}(\mathbf{n}) \frac{\delta T}{T}\left(\mathbf{n}^{\prime}\right)\right\rangle=\frac{1}{4 \pi} \sum_{l=0}^{\infty}(2 l+1) C_{l} P_{l}(\cos \theta),
$$




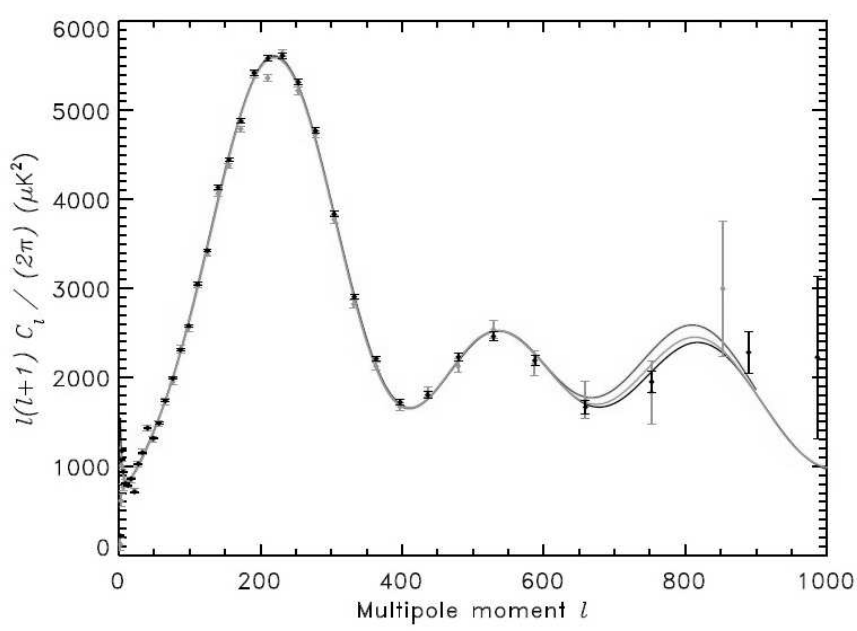

Figure 4: Legendre coefficients for the CMBR temperature correlations, as measured by the WMAP collaboration [5].

and quoting the measured values for $C_{l}$. (See Fig. 4 for recent measurements of these coefficients.)

Perturbations to the Dark Matter density, $\delta \rho_{\mathrm{m}}$, are related to $\delta T / T$ because the temperature fluctuations arise due to the redshift of CMBR photons as they climb out of the gravitational potential wells that are generated by $\delta \rho_{\mathrm{m}}-$ a phenomenon called the Sachs-Wolfe effect. (In a matter-dominated universe, the quantity $\Phi+\delta T / T$ turns out to be a constant along a photon trajectory [3]). Measurements agree well with what is expected theoretically, provided the primordial power spectrum has a simple power-law form $P_{\rho}^{0}(k)=A k^{n_{s}}$, with $n_{s} \simeq 1$.

The choice $n_{s}=1$ is called the Harrison-Zel'dovich (HZ) spectrum, and is special because it corresponds to the case where the dependence of $\Delta_{\rho}^{2}$ is approximately scale invariant for modes which re-enter the horizon during the recent radiation-dominated universe: $\Delta_{\rho}^{2} \propto k^{0}$ for $k>k_{\text {eq }}$ (and so $\Delta_{\rho}^{2} \propto k^{4}$ for $k<k_{\text {eq }}$ ). It also corresponds to scale-invariant fluctuations for the Newtonian gravitational potential, $\Phi$ (defined in more detail for the relativistic case below), when $k<k_{\text {eq. }}$. To see why, notice that $\Phi$ is related to $\rho$ by the Poisson equation - i.e. $\nabla^{2} \Phi=4 \pi G \rho-$ and so the power spectra for $\Phi$ and $\rho$ should be related by $P_{\Phi}(k) \propto P_{\rho}(k) / k^{4}$. Consequently, if $P_{\rho}(k) \propto k^{n_{s}}$ for small $k$, then $P_{\Phi}(k) \propto k^{n_{s}-4}$ and the corresponding dimensionless power spectrum is $\Delta_{\Phi}^{2}(k) \propto$ $k^{3} P_{\Phi}(k) \propto k^{n_{s}-1}$, which is independent of $k$ when $n_{s}=1$.

\section{Evolution of Primordial Fluctuations}

Since inflation provides the past from which the Hot Big Bang later evolves, it is natural to try to compute quantities like $P_{\Phi}^{0}(k)$, assuming they arise from this earlier epoch. To this end it is necessary to follow the evolution of small fluctuations in the inflaton, $\delta \varphi$, as well as the metric, $\delta g_{\mu \nu}$, during and after the inflationary epoch. ${ }^{2}$

\footnotetext{
${ }^{2}$ The discussion here follows the excellent treatment in [3].
} 
The perturbations of the metric, $\delta g_{\mu \nu}$ come in three kinds: scalar, vector and tensor fluctuations, which differ in how they transform under rotations (and so evolve independently of one another at linear order in the fluctuations). After transforming to conformal time, $\hat{\eta}=\int \mathrm{d} t / a$, the scalar perturbations may be written

$$
\delta_{S} g_{\mu \nu}=a^{2}\left(\begin{array}{cc}
2 \phi & \partial_{j} \mathscr{B} \\
\partial_{i} \mathscr{B} & 2 \psi \delta_{i j}+\partial_{i} \partial_{j} \mathscr{E}
\end{array}\right)
$$

while the vector and tensor ones become

$$
\delta_{V} g_{\mu \nu}=a^{2}\left(\begin{array}{cc}
0 & \mathscr{V}_{j} \\
\mathscr{V}_{i} & \partial_{i} \mathscr{W}_{j}+\partial_{j} \mathscr{W}_{i}
\end{array}\right) \quad \text { and } \quad \delta_{T} g_{\mu \nu}=a^{2}\left(\begin{array}{cc}
0 & 0 \\
0 & h_{i j}
\end{array}\right) .
$$

The freedom to perform infinitesimal coordinate transformations allows these functions to be changed, so it is useful to define the following coordinate-invariant combinations:

$$
\begin{aligned}
\Phi & =\phi-\frac{1}{a}\left[a\left(\mathscr{B}-\mathscr{E}^{\prime}\right)\right]^{\prime}, \quad \Psi=\psi+\frac{a^{\prime}}{a}\left(\mathscr{B}-\mathscr{E}^{\prime}\right) \\
\delta \chi & =\delta \varphi-\varphi^{\prime}\left(\mathscr{B}-\mathscr{E}^{\prime}\right), \quad V_{i}=\mathscr{V}_{i}-\mathscr{W}_{i} \quad \text { and } h_{i j}
\end{aligned}
$$

in terms of which all physical inferences can be drawn. Here primes denote differentiation with respect to conformal time, $\hat{\eta}$. Notice that $\Phi, \Psi$ and $V_{i}$ reduce to $\phi, \psi$ and $\mathscr{V}_{i}$ in the gauge choice where $\mathscr{B}=\mathscr{E}=\mathscr{W}_{i}=0$, and so $\Phi$ is the relativistic generalization of the Newtonian potential.

Exercise 4: Show that the combinations given in eqs. (2.27) are invariant under infinitesimal coordinate transformations: $\delta \varphi=\xi^{\mu} \partial_{\mu} \varphi$ and $\delta g_{\mu \nu}=\xi^{\lambda} \partial_{\lambda} g_{\mu v}+\partial_{\mu} \xi^{\lambda} g_{\lambda v}+$ $\partial_{\nu} \xi^{\lambda} g_{\lambda \mu}$

These functions are evolved forward in time by linearizing the relevant field equations:

$$
\square \varphi-V^{\prime}(\varphi)=0 \quad \text { and } \quad R_{\mu v}-\frac{1}{2} R g_{\mu \nu}=\frac{T_{\mu \nu}}{M_{p}^{2}},
$$

and provided we use the invariant stress-energy perturbations,

$$
\begin{aligned}
\delta \mathscr{T}^{0}{ }_{0} & =\delta T^{0}{ }_{0}-\left[t^{0}{ }_{0}\right]^{\prime}\left(\mathscr{B}-\mathscr{E}^{\prime}\right), \\
\delta \mathscr{T}^{0}{ }_{i} & =\delta T^{0}{ }_{i}-\left[t^{0}{ }_{0}-\frac{1}{3} t^{k}{ }_{k}\right] \partial_{i}\left(\mathscr{B}-\mathscr{E}^{\prime}\right), \\
\delta \mathscr{T}^{i}{ }_{j} & =\delta T^{i}{ }_{j}-\left[t^{i}{ }_{j}\right]^{\prime}\left(\mathscr{B}-\mathscr{E}^{\prime}\right),
\end{aligned}
$$

(where $t^{\mu}{ }_{v}$ denotes the background stress-energy), the results can be expressed purely in terms of the gauge-invariant quantities, eqs. (2.27).

The equations which result show that in the absence of vector stress-energy perturbations, the vector perturbation $V_{i}$ is not sourced, and decays very rapidly in an expanding universe, allowing it to be henceforth ignored. Similarly, in the absence of off-diagonal stress-energy perturbations it is also generic that $\Psi=\Phi$. 


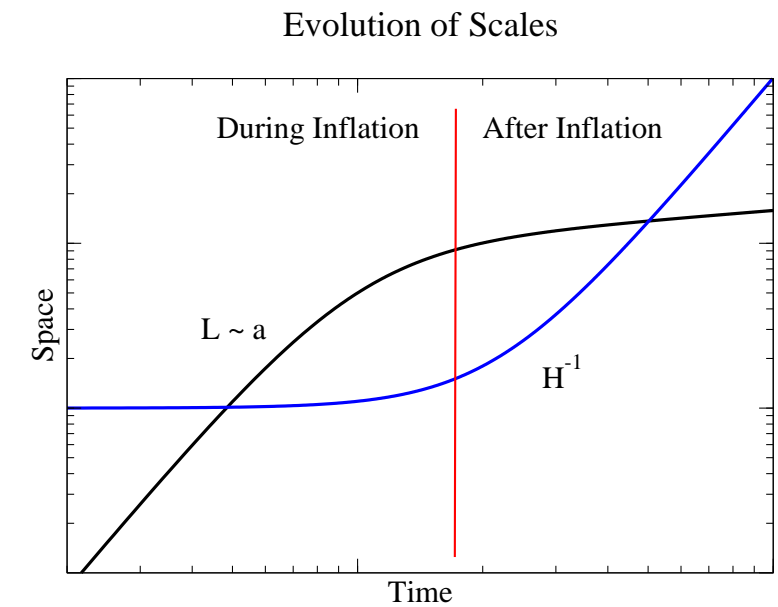

Figure 5: A sketch of the relative growth of physical scales, $L(t)$, (in black) and the Hubble length, $H^{-1}$, (in blue) during and after inflation.

The equations which govern the evolution of tensor modes then become (after Fourier transforming)

$$
\ddot{h}_{i j}+3 H \dot{h}_{i j}+\frac{k^{2}}{a^{2}} h_{i j}=0,
$$

while the scalar fluctuations similarly reduce to

$$
\begin{gathered}
\delta \ddot{\chi}+3 H \delta \dot{\chi}+\frac{k^{2}}{a^{2}} \delta \chi+V^{\prime \prime}(\varphi) \delta \chi-4 \dot{\varphi} \dot{\Phi}+2 V^{\prime}(\varphi) \Phi=0 \\
\text { and } \quad \dot{\Phi}+H \Phi=\frac{\dot{\varphi}}{2 M_{p}^{2}} \delta \chi
\end{gathered}
$$

which shows that it is the time-dependence of the background configurations which forces $\delta \chi$ and $\Phi$ to mix with one another. The homogeneous background fields in these expressions themselves satisfy the equations

$$
\ddot{\varphi}+3 H \dot{\varphi}+V^{\prime}(\varphi)=0 \quad \text { and } \quad 3 M_{p}^{2} H^{2}=\frac{1}{2} \dot{\varphi}^{2}+V(\varphi) .
$$

Exercise 5: Derive eq. (2.30). (Hint: use conformal time, $\hat{\eta}=\int \mathrm{d} t / a$.)

\section{Scalar Perturbations}

The character of the solutions of these equations depends strongly on the size of $k / a$ relative to $H$, since this dictates the extent to which the frictional terms can compete with the spatial derivatives. For instance, an approximate form for the two independent solutions for $\delta \chi$ that applies when $k / a \gg H$ is given by damped oscillations

$$
\delta \chi_{k} \propto \frac{1}{a \sqrt{k}} \exp \left[ \pm i k \int^{t} \frac{\mathrm{d} t^{\prime}}{a\left(t^{\prime}\right)}\right] .
$$


A similar expression in the limit $k / a \ll H$ is also obtainable during inflation by using the slow-roll approximation, for which we neglect $\delta \ddot{\chi}, \ddot{\varphi}$ and $\dot{\Phi}$. In this case the approximate non-decaying solution to

$$
3 H \delta \dot{\chi}+V^{\prime \prime}(\varphi) \delta \chi+2 V^{\prime}(\varphi) \Phi \simeq 0 \quad \text { and } \quad 2 M_{p}^{2} H \Phi \simeq \dot{\varphi} \delta \chi,
$$

is given (after Fourier transformation) by

$$
\delta \chi_{k} \simeq C_{k} \frac{V^{\prime}(\varphi)}{V(\varphi)} \quad \text { and } \quad \Phi_{k} \simeq-\frac{C_{k}}{2}\left(\frac{V^{\prime}(\varphi)}{V(\varphi)}\right)^{2} .
$$

where $C_{k}$ is a (potentially $k$-dependent) constant of integration.

Exercise 6: Verify that eqs. (2.35) satisfy eqs. (2.34).

The transition between these two qualitatively different kinds of behaviour occurs when $k / a \simeq$ $H$. When the product $a H$ is shrinking (such as during radiation and matter domination) the condition $k / a=H$ is satisfied for successively smaller values of $k$ (longer wavelengths) as time goes on. Conversely, when $a H$ grows (as during inflation) it is the larger values of $k$ (shorter wavelengths) which satisfy $k / a=H$ at later times. A typical mode with wavelength $\lambda=2 \pi a / k$ smaller than the Hubble length, $H^{-1}$, during inflation is therefore stretched until it eventually becomes larger than $H^{-1}$, at the epoch of horizon exit. It continues to grow compared with the Hubble scale until inflation ends, after which it is $H^{-1}$ which grows faster than $\lambda$ (see Fig. 5).

During inflation, the modes of interest initially start off with $k / a \gg H_{I}$, and any initial oscillations are efficiently damped by the exponential factor $1 / a \propto e^{-H_{I} t}$ in eq. (2.33), removing all memory of the initial configuration. However, eventually $k / a$ falls far enough that the mode 'leaves the horizon' to satisfy $k / a \ll H_{I}$. At this point the growing solution, eq. (2.35), starts to dominate. During inflation the growth of this solution is slow, because $\delta \chi_{k} \propto \sqrt{\varepsilon}$ and $\Phi_{k} \propto \varepsilon$, where the slowroll parameter, $\varepsilon$, of eq. (2.9), is necessarily small. This evolution need no longer remain small once inflation ends, but at this point the slow-roll assumption used to derive the solution, eq. (2.35) breaks down.

\section{Source of Fluctuations}

The primordial fluctuation amplitude derived in this way depends on the integration constants $C_{k}$, which are themselves set by the initial conditions for the fluctuation at horizon exit, during inflation. But why should this amplitude be nonzero given that all previous evolution is strongly damped, as in eq. (2.33)? The result remains nonzero (and largely independent of the details of earlier evolution) because quantum fluctuations in $\delta \chi$ continually replenish the perturbations long after any initial classical configurations have damped away.

The starting point for the calculation of the amplitude of scalar perturbations is the observation that the inflaton and metric fields whose dynamics we are following are quantum fields, not classical ones. For instance, for spatially-flat spacetimes the linearized inflaton field, $\delta \chi$, is described by the operator

$$
\delta \chi(x)=\int \frac{\mathrm{d}^{3} k}{(2 \pi)^{3}}\left[c_{k} u_{k}(t) e^{i \mathbf{k} \cdot \mathbf{r} / a}+c_{k}^{*} u_{k}^{*}(t) e^{-i \mathbf{k} \cdot \mathbf{r} / a}\right]
$$


where we expand in a basis of eigenmodes of the scalar field equation in the background metric, $u_{k}(t) e^{i \mathbf{k} \cdot \mathbf{x}}$, labelled by the co-moving momentum $\mathbf{k}$. For constant $H$ the time-dependent mode functions are

$$
u_{k}(t) \propto \frac{H}{k^{3 / 2}}\left(i+\frac{k}{a H}\right) \exp \left(\frac{i k}{a H}\right),
$$

which reduces to the standard flat-space form (up to a slowly-varying phase), $u_{k}(t) \propto a^{-1} k^{-1 / 2} e^{-i k t / a}$, when $k / a \gg H$. The quantities $c_{k}$ and their adjoints $c_{k}^{*}$ are annihilation and creation operators, which define the adiabatic vacuum state, $|\Omega\rangle$, through the condition $c_{k}|\Omega\rangle=0$ (for all $\mathbf{k}$ ).

The $\delta \chi$ auto-correlation function in this vacuum, $\left\langle\delta \chi(x) \delta \chi\left(x^{\prime}\right)\right\rangle$, describes the quantum fluctuations of the field amplitude in the quantum ground state. Assuming these quantum fluctuations get decohered sometime during or after inflation in an as-yet poorly understood way (for preliminary discussions see ref. [9]), sometime between horizon exit and horizon re-entry these quantum fluctuations eventually become converted into classical statistical fluctuations of the classical field, $\varphi$, about its spatial mean, by an amount of order $\left|\delta \chi_{k}\right| \sim\left[\left\langle\delta \chi_{k} \delta \chi_{-k}\right\rangle\right]^{1 / 2} \propto\left|u_{k}(t)\right|$. Although the details of this decoherence remain unclear, for observational purposes all that matters is that the classical variance of these statistical fluctuations is well-described by the corresponding quantum auto-correlations - a property that is expected to be a good approximation given the kinds of 'squeezed' quantum states which are generated during inflation $[11,3]$.

Evaluating $\delta \chi_{k} \sim u_{k}$ at $t_{\text {he }}$ (where $k=a H$ ) and equating the result to the fluctuation of eq. (2.35) allows the integration constant in this equation to be determined

$$
C_{k}=u_{k}\left(t_{\mathrm{he}}\right)\left(\frac{V}{V^{\prime}}\right)_{\varphi_{\mathrm{he}}},
$$

where both $t_{\text {he }}$ and $\varphi_{\text {he }}=\varphi\left(t_{\text {he }}\right)$ implicitly depend on $k$. Using this to compute $\Phi_{k}$ in eq. (2.35) then gives, near the end of inflation

$$
\Phi_{k}\left(t_{\mathrm{end}}\right)=-\frac{1}{2} u_{k}\left(t_{\mathrm{he}}\right)\left(\frac{V}{V^{\prime}}\right)_{\varphi_{\mathrm{he}}}\left(\frac{V^{\prime}}{V}\right)_{\varphi_{\mathrm{end}}}^{2}=-\varepsilon\left(t_{\mathrm{end}}\right)\left(\frac{u_{k}}{\sqrt{2 \varepsilon} M_{p}}\right)_{t_{\mathrm{he}}} .
$$

Notice that the factors depending on $t_{\text {end }}$ are generically $O(1)$ if taken at the end of inflation, and do not affect the $k$-dependence of the result.

\section{Post-Inflationary Evolution}

For the case of single-field inflation discussed here, the subsequent post-inflationary evolution of the fluctuation $\Phi$ - which is what governs both $\delta \rho_{\mathrm{m}}$ and $\delta T / T$ - can be solved quite generally (in single-field slow-roll models), so long as $k / a \ll H$. This is because it can be shown that when $k \ll a H$ the quantity

$$
\zeta=\Phi+\frac{2}{3}\left(\frac{\Phi+\dot{\Phi} / H}{1+w}\right)=\frac{1}{3(1+w)}\left[(5+3 w) \Phi+\frac{2 \dot{\Phi}}{H}\right]
$$

is conserved, $\dot{\zeta} \simeq 0$, where $w \equiv p / \rho$ is not assumed to be constant. This is a very powerful result because it can be used to evolve fluctuations using $\zeta\left(t_{i}\right)=\zeta\left(t_{f}\right)$, assuming only that they 
involve a single scalar field, and that the modes in question are well outside the horizon: $k / a \ll H$. Furthermore, although $\dot{\Phi}$ in general becomes nonzero at places where $w$ varies strongly with time, this time dependence quickly damps due to Hubble friction for modes outside the Hubble scale. We may therefore neglect the dependence of $\zeta$ on $\Phi$ provided we restrict $t_{i}$ and $t_{f}$ to epochs during which $w$ is roughly constant. This allows the expression $\zeta\left(t_{i}\right)=\zeta\left(t_{f}\right)$ to be simplified to

$$
\Phi_{f}=\frac{1+w_{f}}{1+w_{i}}\left(\frac{5+3 w_{i}}{5+3 w_{f}}\right) \Phi_{i}
$$

where $w_{i}=w\left(t_{i}\right)$ and $w_{f}=w\left(t_{f}\right)$, implying in particular $\Phi_{f}=\Phi_{i}$ whenever $w_{i}=w_{f}$.

Exercise 7: Use the conservation of $\zeta$ to show that (when $k / a \rightarrow 0$ ), $\Phi_{\mathrm{m}}=\frac{9}{10} \Phi_{\mathrm{rad}}$ for modes evaluated well before and well after the transition from radiation to matter domination.

Exercise 8: Show that $1+w \simeq \dot{\varphi}^{2} / V \simeq \frac{2}{3} \varepsilon$ during single-field slow-roll inflation, and use this with eq. (2.41) to provide an alternate derivation of eq. (2.39). That is, show that (when $k / a \rightarrow 0$ ), $\Phi_{f} / \Phi_{i}=\varepsilon_{f} / \varepsilon_{i}$ for times $t_{i}$ and $t_{f}$ both well within the inflationary epoch.

To infer the value of $\Phi$ in the later Hot Big Bang era we choose $t_{i}$ just after horizon exit (where $w_{i} \simeq-1+\frac{2}{3} \varepsilon_{\mathrm{he}}-$ see Exercise 8 ). $t_{f}$ is then chosen in the radiation dominated universe (where $w_{f}=\frac{1}{3}$ ), either just before horizon re-entry for the mode of interest, or just before the transition to matter domination, whichever comes first. Eqs. (2.41) and (2.39) then imply

$$
\Phi_{f} \simeq\left(\frac{6 \Phi}{\varepsilon}\right)_{\mathrm{he}} \simeq-\left(\frac{3 \sqrt{2} u_{k}}{\sqrt{\varepsilon} M_{p}}\right)_{\mathrm{he}} .
$$

Using this in the definition of the dimensionless power spectrum for $\Phi, \Delta_{\Phi}^{2}=k^{3} P_{\Phi} /\left(2 \pi^{2}\right)$, then leads to

$$
\Delta_{\Phi}^{2}(k) \sim k^{3}\left|\Phi_{k}\left(t_{f}\right)\right|^{2} \sim \frac{\left|k^{3 / 2} u_{k}\left(t_{\mathrm{he}}\right)\right|^{2}}{\varepsilon\left(\varphi_{\mathrm{he}}\right) M_{p}^{2}} \sim\left(\frac{H^{2}}{\varepsilon M_{p}^{2}}\right)_{\varphi_{\mathrm{he}}} \sim\left(\frac{V}{\varepsilon M_{p}^{4}}\right)_{\varphi_{\mathrm{he}}} .
$$

Once the order-unity factors are included from a more detailed calculation one finds

$$
\Delta_{\Phi}^{2}(k)=\frac{k^{3} P_{\Phi}(k)}{2 \pi^{2}}=\left(\frac{H^{2}}{8 \pi^{2} M_{p}^{2} \varepsilon}\right)_{\text {he }}=\left(\frac{V}{24 \pi^{2} M_{p}^{4} \varepsilon}\right)_{\text {he }},
$$

We see that because it is $V / \varepsilon$ which controls the amplitude of density fluctuations, measurements of this amplitude provide information about the energy scale which dominates the universe during inflation. For the purposes of comparison it is convenient to define [7] the quantity $\delta_{H}(k)$ by $\delta_{H}^{2}=(4 / 25) \Delta_{\Phi}^{2}(k)$, since the observed amplitude of large-angle temperature fluctuations requires

$$
\delta_{H}(\hat{k})=1.91 \times 10^{-5},
$$


when evaluated at $k=\hat{k} \sim 7.5 a_{0} H_{0}$. In terms of $V$ this implies

$$
\left(\frac{V}{\varepsilon}\right)^{1 / 4}=6.6 \times 10^{16} \mathrm{GeV}
$$

The smaller $\varepsilon$ becomes, the smaller a potential energy is required, and for $\varepsilon \sim 0.01$ we have $V \sim 2 \times$ $10^{15} \mathrm{GeV}$. This is remarkably close to the scale where the couplings of the three known interactions appear to unify, and may indicate a connection between inflation and more exotic physics like the physics of Grand Unification. ${ }^{3}$

\section{Spectra}

We now compute in more detail what eq. (2.44) implies for the $k$-dependence of the primordial fluctuation spectrum. Notice to this end that to first approximation the size of $\Delta^{2}(k)$ is set by $H$ and $\varepsilon$ and does not depend explicitly on $k$ at all. This observation underlies the approximate scaleinvariance of the primordial power spectrum which inflation predicts for the later universe.

However, inflation does predict a weak $k$-dependence for the right-hand-side because it must be evaluated for $\varphi=\varphi_{\text {he }}$, defined as the value taken by $\varphi(t)$ at the epoch $t_{\text {he }}=t_{\text {he }}(k)$ when the co-moving wavelength $k$ of interest is just exiting the Hubble length $k=a\left(t_{\text {he }}\right) H\left(t_{\text {he }}\right)$. It is this $k$ dependence of the horizon-exit time which introduces small deviations from scale invariance into the predicted power spectrum.

To quantify this more precisely, recall that in earlier sections a successful phenomenological parametrization of the density power spectrum was given by $P_{\rho}(k) \propto k^{n_{s}}$, and that this choice implies the primordial gravitational power spectrum satisfies $\Delta_{\Phi}^{2}=A k^{n_{s}-1}$. Deviations from scale invariance may be computed by evaluating

$$
n_{s}-\left.1 \equiv \frac{\mathrm{d} \ln \Delta_{\Phi}^{2}}{\mathrm{~d} \ln k}\right|_{\mathrm{he}},
$$

and using the condition $k=a H$ (and the constancy of $H$ during inflation) to write $\mathrm{d} \ln k=H \mathrm{~d} t$. Since the right-hand side of eq. (2.44) depends on $\varphi$, it is convenient to use the slow-roll equations, eq. (2.8) to further change variables from $t$ to $\varphi$ : $\mathrm{d} t=-\left(3 H / V^{\prime}\right) \mathrm{d} \varphi$, and so

$$
\frac{\mathrm{d}}{\mathrm{d} \ln k}=-M_{p}^{2}\left(\frac{V^{\prime}}{V}\right) \frac{\mathrm{d}}{\mathrm{d} \varphi}
$$

These expressions allow the derivation of the following relation between $n_{s}$ and the slow-roll parameters, $\varepsilon$ and $\eta$ :

$$
n_{s}-1=-6 \varepsilon+2 \eta
$$

where the right-hand side is evaluated at $\varphi=\varphi_{\text {he }}$.

Notice that this prediction for the spectral index makes $n_{s}<1$ for both the large- and smallfield inflation models considered above. Recall that for large-field models (with $V=\frac{1}{n} \lambda^{2} \varphi^{n}$ ) we

\footnotetext{
${ }^{3}$ Of course, $V$ can be much smaller if $\varepsilon$ is smaller as well, or if primordial fluctuations come from another source. For instance generating primordial fluctuations from $\mathrm{TeV}$ scale inflation [12] would require $\varepsilon \simeq 10^{-55}$.
} 
had $\varepsilon=\frac{1}{2} n^{2}\left(M_{p} / \varphi\right)^{2}>0$ and $\eta=2(1-1 / n) \varepsilon$ and so $-6 \varepsilon+2 \eta=-(2+4 / n) \varepsilon<0$. On the other hand, for small-field models (where $V=M_{I}^{4}-\mu^{2} \varphi^{2}+\cdots$, we had $\eta \leq 0$ because we work near $\varphi=0$, which is a maximum of $V$. Since in this case $\eta \leq 0$ and $\varepsilon \geq 0$, they necessarily both make negative contributions to $-6 \varepsilon+2 \eta$.

Observational inferences of $n_{s}$ from the detailed shape of the CMBR temperature fluctuation spectrum now give a central value of $n_{s}=0.951 \pm 0.016$, with $n_{s}=1$ beginning to be disfavoured [5] (assuming no tensor fluctuations - see Fig. 6 below).

\section{Tensor Fluctuations}

A very similar story goes through for the tensor fluctuations that are generated by quantum fluctuations, although in this case these fluctuations have not yet been observed. Just like for scalar fluctuations, for each propagating mode these are generated with amplitude $H /(2 \pi)$, but unlike for scalar modes it is not necessary for the inflaton to mix with a gravitational mode to obtain an observable effect, and so the power spectrum for tensor perturbations does not share the singular factor of $1 / \varepsilon$.

Similar arguments to those given above then lead to the following dimensionless tensor power spectrum

$$
\Delta_{T}^{2}(k)=\frac{8}{M_{p}^{2}}\left(\frac{H}{2 \pi}\right)^{2}=\frac{2 V}{3 \pi^{2} M_{p}^{4}} .
$$

As expected, this differs from the scalar power spectrum by depending only on the value of $V$ and not also on the slow-roll parameter $\varepsilon$. Consequently, should both scalar and tensor modes be measured, a comparison of their amplitudes provides a direct measure of the slow-roll parameter $\varepsilon$. A more precise version of this comparison can be phrased in terms of a parameter $r$, which is defined as a ratio of the scalar and tensor power spectra

$$
r \equiv \frac{\Delta_{T}^{2}}{\Delta_{\Phi}^{2}}=16 \varepsilon
$$

The failure to detect these perturbations to date places a relatively weak upper limit: $r<0.30$ (95\% CL) [5], or $\varepsilon<0.02$.

Once tensor modes are detected, more information can be found from its power spectrum as a function of $k$. In particular, the tensor spectral index, $n_{T}$, is defined by

$$
n_{T} \equiv \frac{\mathrm{d} \Delta_{T}^{2}}{\mathrm{~d} \ln k}=-2 \varepsilon=-\frac{r}{8},
$$

where the last equality evaluates the derivative by changing variables from $k$ to $\varphi$. Again the result is to be evaluated at the epoch when observable modes leave the horizon during inflation, $\varphi=\varphi_{\text {he }}$.

\section{Implications for the CMBR}

In summary, quantum fluctuations generated during slow-roll inflation provide a natural source for the small temperature variations visible in the CMBR, which also appear to have seeded galaxy 

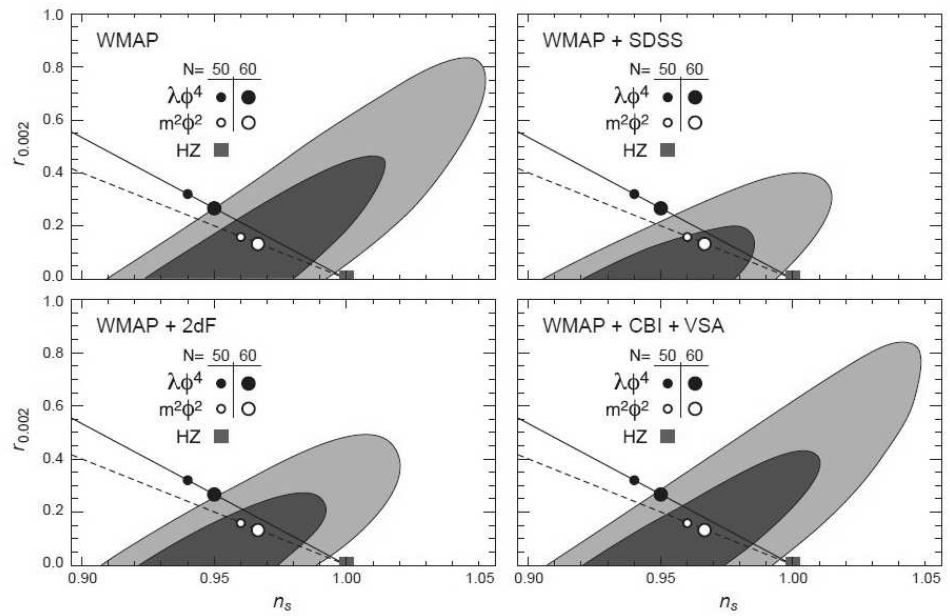

Figure 6: Best fits to the ratio of tensor to scalar fluctuations and spectral index from WMAP, compared with the predictions of various inflationary models [5].

formation. Furthermore, single-field slow-roll inflation makes the following detailed, yet successful, predictions for the form of the primordial fluctuation spectrum which are inferred from the large-angle properties of temperature fluctuations for the CMB photons.

Gaussian Fluctuations: Because inflation requires such a slow roll, the fluctuations in the inflaton field are very weakly coupled to one another. This turns out to imply that the late-time density fluctuations are predicted obey Gaussian statistics. To date no non-Gaussian correlations have been seen in the CMBR (more about this below).

In-Phase Perturbations: The process whereby fluctuations freeze while they are outside of the Hubble scale, and then begin to evolve again once liberated by re-entering the Hubble scale during our much-later epoch, implies these fluctuations all enter the horizon in phase. Being in phase allows for the coherent peaks and valleys of the $C_{l}$ 's which are seen in Fig. 4, and is not predicted by many of the alternative theories of the primordial density fluctuations (such as their production by cosmic strings or other defects).

Adiabatic Perturbations: The process of re-entry of fluctuations, after their having been frozen over long periods while outside the Hubble scale implies the fluctuations enter the horizon at rest. This is crucial for determining the $l$ value for the position of the first peak in the CMB spectrum, and is verified by the observations that this peak occurs at $l \approx 200$. This prediction need no longer hold if more than one scalar field is involved in inflation.

Almost Scale Invariant Spectrum: Inflation predicts a spectrum of fluctuations which is close to, but not exactly, scale invariant. For instance if $N_{e} \sim 60$ implies $\varepsilon \sim|\eta| \sim 1 / 60$, then the deviation $n_{s}-1$ should be a few percent. Current measurements prefer such a deviation, with an accuracy that is on the verge of excluding an interesting part of the parameter space of inflationary models.

Scalar to Tensor Ratio: The same parameters which determine the scalar fluctuation spectrum also predict the tensor fluctuation properties. A good test of the theory is provided once tensor 
modes are observed, because the tensor and scalar fluctuations are characterized by 4 observable quantities (amplitude and spectral index for both scalar and tensor modes), and the theory predicts these in terms of three parameters: $H, \varepsilon$ and $\eta$. The present status of these observational tests is given in Fig. 4 [13, 5, 14].

\subsection{Problems With Inflation?}

The general idea of there being an epoch of accelerated expansion as a solution for the horizon and flatness problems is very simple and attractive, and the additional feature that it also accounts for the primordial spectrum of temperature fluctuations is quite compelling. Nevertheless some conceptual problems remain with inflation, and are mostly associated with our ignorance about the physics which governs the enormously high energies which inflation could probe. Many of these potential problems can be phrased in terms of naturalness issues that arise once specific models having an inflationary dynamics are made (such as the single-field slow-roll models examined in earlier sections).

Some of the main concerns of this sort are now listed, with an eye to seeing how the next section's contact with string theory might help.

Initial Conditions: As was seen in the models studied above, inflation tends to arise only for particular kinds of initial conditions for the fields. For instance, small-field inflation requires the initial value of $\varphi$ to be very close to a maximum of the potential, and it is not clear why the universe should start off in this region. (By contrast, large-field inflation occurs over a broader range of initial conditions, but relies on having reliably-calculable potentials for large field values, $\varphi \gg M_{p}$.) Relying on special initial conditions is uncomfortable because inflation was invented to provide a physical explanation for the origin of the unnatural initial conditions which are required for the success of the Hot Big Bang. If we are happy to choose special initial conditions to obtain inflation, why not instead simply choose the special conditions required by the Big Bang?

Special Potentials: The success of the inflationary models studied above relies on the potential energy being quite flat, since $V^{\prime} / V$ and $V^{\prime \prime} / V$ must be suppressed to make the slow-roll parameters $\varepsilon$ and $\eta$ sufficiently small. But it is difficult to make such choices for a scalar potential stable against quantum corrections, since they are very sensitive to the microscopic particle content of the theory which underlies the inflationary model. It remains to be seen if this remains a problem once the best theories we have for the relevant microscopic physics (like string theory) are used to try to produce inflation.

Reheating: Since inflation ruthlessly 'inflates away' any previously existing particles and energy, it can only precede the Hot Big Bang epoch if it comes with a mechanism for transferring energy into the heating of the contents of the observable post-inflationary universe. How is this energy transfer accomplished? [15]

Predicting in the Multiverse: In general causality forbids a completly homogeneous field evolution, with fields in causally-disconnected regions of spacetime evolving independent of one another. This means that we should only imagine the above inflationary picture for $\varphi$ describing one 
of these regions, with other regions being described by slightly different initial conditions (and possibly also different scalar potentials, if the couplings of the inflaton is related to the values taken of other fields). But since each region evolves dramatically differently depending on whether it inflates or not, how does one make predictions in such a diverse universe? One might expect that inflation exponentially rewards those parts of the universe that choose the initial conditions leading to inflation, even if these conditions are comparatively improbable, because of the exponential growth of the volume of the region which does so. Does this contain the seeds of a probabilistic understanding of the properties of the later universe[16, 17]?

It remains to be seen how serious each of these problems really is, but there is considerable motivation to understand them in some detail given the simplicity of the inflationary understanding of the large-scale features of the observed CMBR temperature fluctuations.

\section{Towards String Inflation}

The last section closed with a list of potential problems for inflation, whose resolution requires an understanding of the physics at the potentially enormous energies - possibly as large as $M_{I} \lesssim$ $10^{15} \mathrm{GeV}$ - at which inflation can take place. What guidance can particle physics provide as to what this physics might be?

Since the energies involved could be not much lower than the Planck scale, $M_{p}=(8 \pi G)^{-1 / 2} \sim$ $10^{18} \mathrm{GeV}$, it is not unreasonable to look to theories including quantum gravity when searching for this guidance. At present, the theory which provides the best-developed and best-motivated framework of quantum gravity is string theory, making this a natural laboratory for seeking inflationary dynamics. This section describes some recent progress along these lines, with several possible inflationary mechanisms being identified. Since the target audience is not string theorists, the description will be in broad brush-strokes rather than fine detail, with an eye towards the broader inflationary lessons that are being learned.

\section{What One Might Hope to Learn}

Before launching into a lengthy technical preamble to building inflationary scenarios within string theory, it is worth first stating why one might be interested in doing so in the first place. (See ref. [18] for reviews of string-based inflation.) After all, present observations can just barely differentiate amongst the simplest single-field slow-roll models, so one might reasonably ask why bother building the inevitably more baroque string models. The thinking is that string theory potentially can provide new insight into several issues in inflationary cosmology.

Robustness of Inferences: Much of the observational evidence for inflation rests on it being the source of the primordial fluctuations, but its success in doing so is largely based on the predictions of very simple single-field models. But is the single-field approximation too simple given the many fields which typically arise in fundamental theories? Even if not, if microscopic physics is being stretched by inflation up to cosmological distances, can the physics of much smaller scales be similarly stretched [19], and so influence inflationary predictions in unexpected ways? If so, then the 
observational evidence for inflation would be undermined by this introduction of an uncontrollable theoretical error into its predictions [20]. Such questions can be tested in string theory, with current evidence supporting the robustness of the predictions of simple inflationary models [21].

Validity of Approximations: Single field models often rely for their validity on approximations whose validity cannot be properly established without better understanding the high-energy limit of the theory. For instance, for large-field inflationary models successful inflation relies on fields taking large values, $\varphi \gg M_{p}$, and this is also typically required to obtain observably large primordial tensor fluctuations [22]. But whether such large fields make sense depends on properly understanding the shape of the scalar potential for such large field values. String theory can shed light on this by providing a physical interpretation for the inflaton (such as being the distance between two branes [23]), and so can identify upper limits in its range (such as it not being larger than the size of the extra dimensions in which the branes move [24]). Detailed arguments like these have led to the conjecture that observable primordial tensor fluctuations may be unlikely to be obtained from string theoretic inflation [26].

Initial Conditions and Naturalness: How unusual is inflation? Inflationary models can require comparatively flat potentials and special initial conditions, but an understanding of how special these are requires a broader understanding of the shape of the scalar potential, and of the likely initial conditions before inflation, which only a fundamental theory like string theory can ultimately provide.

Reheating: As noted above, the energy density which drives inflation must ultimately get transformed to heat for the later Hot Big Bang. Just as having a warm house in the winter requires both a good furnace and good insulation, successful reheating after inflation requires two things: (i) a sufficiently strong coupling between the inflaton and the ordinary Standard Model particles we now see around ourselves; and (ii) the absence of too strong couplings between the inflaton and any other, currently unobserved, degrees of freedom. It is clear that the second part of this question cannot be properly addressed without knowing the full theory describing all the degrees of freedom which are relevant at the energies available after inflation.

Mind Broadening: Simple inflationary models make simplifying assumptions which need not be true, but which tend to guide our search for models. Embedding inflation into string theory has already exposed some of these assumptions, and may yet expose more. For instance, it is often assumed that the inflaton field remains around after inflation ends and still appears in the low-energy theory describing the later Hot Big Bang epoch. However if the inflaton were the separation between a brane and antibrane which mutually annihilate at inflation's end [24, 25], then the inflaton does not even make sense as a field in the later universe. Similarly, although inflation now seems compelling to us in the context of field theory, perhaps string theory provides novel alternative ways [27] to solve the initial condition problems which inflation was originally invented to solve. 


\subsection{General Framework}

String theory is much more complicated than the simple inflaton models discussed above, involving a potentially infinite number of particle types (string modes), moving in more than 4 dimensions. The space of vacua which is allowed is only partially understood, but that part which is already well explored shows that it is incredibly vast and diverse - involving many possible vacuum values for many possible low-energy fields. (See ref. [28] for textbook descriptions of string theory, and [29] for useful reviews.)

Part of this complexity can be traced to there being a large number of scales in string theory, and for inflationary purposes there are at least three which are very important: the string scale, $M_{s}$; the compactification - or Kaluza-Klein $(\mathrm{KK})$ - scale(s), $M_{c}$; and the inflationary scale, $M_{I}$ (and so also $H_{I} \sim M_{I}^{2} / M_{p}$ ). For strings moving in 10D Minkowski space, $M_{s}$ characterizes the mass splitting among generic string modes. $M_{c}$ describes the mass splitting within each string mode when it is placed in a non-trivial background, such as when all but 4 of the dimensions are compactified. For simple geometries characterized by a single length scale, $\ell,(e . g$. a curvature radius, or a volume, $\mathscr{V}_{n}=\ell^{n}$ ), the compactification scale is of order $M_{c} \sim 1 / \ell$. The 4D Planck mass is not an independent scale because it is calculable in terms of the others.

Much of what is known in string theory is restricted to the case $M_{c} \ll M_{s}$, since in this case the effective theory describing energies $E \ll M_{S}$ is given by a higher-dimensional (usually 10 or 11 dimensional) supergravity. If all but 4 of the dimensions are compactified at similar scales, then the physics of energies $E \ll M_{c}$ is described by some sort of 4D effective theory. The 4D Planck scale is typically of order $M_{p} \sim g_{s}^{-1} M_{s}\left(M_{s} / M_{c}\right)^{3} \gg M_{s}$, where $g_{s} \ll 1$ is the string coupling (which in string theory is related to the value of one of the background scalar fields). The field content and symmetries (like supersymmetry) of this low-energy 4D theory depend on the details of the kind of higher-dimensional supergravity, and of its compactification, that is under consideration. In what follows it is always assumed that $M_{c} \ll M_{S}$.

The complexity of an inflationary model in string theory depends crucially on how large is the inflationary Hubble scale, $H_{I} \sim M_{I}^{2} / M_{p}$, compared with both $M_{s}$ and $M_{c}$.

- If $M_{s} \lesssim H_{I}$ then inflation is an intrinsically stringy phenomenon. It is stringy because the time-dependence of the background geometry is sufficient to produce particles having masses up to $O\left(H_{I}\right)$, and this includes nontrivial string modes by assumption. In this case inflation can only be convincingly demonstrated by working with all of the complexity of string theory.

- If $M_{c} \ll H_{I} \ll M_{s}$, then inflation can be described within the effective higher-dimensional field theory, without requiring all the stringy bells and whistles. However in this regime all of the extra-dimensional physics is important, and one is seeking solutions to the full higher-dimensional supergravity equations.

- If $H_{I} \ll M_{c} \ll M_{s}$, then inflation can be intrinsically 4-dimensional, since the energies available to be pair-produced by the time-dependent geometry are generically not high enough to excite any of the KK modes associated with the existence of the extra dimensions. 
Most of the inflationary models proposed to date ${ }^{4}$ are formulated within the last of these categories, with $H_{I} \ll M_{c} \ll M_{s}$, since in this case the problem reduces to searching for time-dependent inflating solutions to the effective 4D field equations. Because these models are being constructed in an explicitly 4D limit, we should not be surprised to find them to share many features of 4D inflationary models, and this is indeed what is found. Of more interest is finding those ways in which inflation differs when the field theory in which it is found arises as a low energy 4D effective theory in string theory, and a few of the known examples of this will be discussed.

\subsection{Multiple Scalars}

Although inflation asks only for one scalar field to be the inflaton, it is a generic feature of string vacua that their low-energy limit contains more than one scalar field. This opens up the possibility that more than one of these fields plays an inflationary role, and so suggests re-examining slow-roll inflation in multi-field models.

\section{Hybrid Inflation}

A useful starting point for multi-scalar inflationary models is Hybrid Inflation [31]. In its simplest form this corresponds to the following action for two scalar fields, $\varphi$ and $\chi$,

$$
S=-\int \mathrm{d}^{4} x \sqrt{-g}\left[\frac{M_{p}^{2}}{2} R+\frac{1}{2} \partial^{\mu} \varphi \partial_{\mu} \varphi+\partial^{\mu} \chi \partial_{\mu} \chi+V(\varphi, \chi)\right],
$$

with scalar potential

$$
V(\varphi, \chi)=\frac{m^{2}}{2} \varphi^{2}+\frac{\lambda^{2}}{4} \varphi^{4}+\frac{g^{2}}{4}\left(\chi^{2}-v^{2}\right)^{2}+\frac{h^{2}}{4} \varphi^{2} \chi^{2}
$$

Here $\lambda, g$ and $h$ are dimensionless, real coupling constants and an additive constant has been chosen to ensure that $V=0$ when evaluated at the potential's global minimum, which is situated at $\varphi=0$ and $\chi=v$.

For inflationary purposes our interest is in the case where the dimensionful constants satisfy $0 \leq m \ll g v$, and where $\varphi$ starts out very large. The derivatives of the potential are

$$
V_{, \varphi}=\varphi\left[m^{2}+\lambda^{2} \varphi^{2}+\frac{h^{2}}{2} \chi^{2}\right] \quad \text { and } \quad V_{, \chi}=\chi\left[g^{2}\left(\chi^{2}-v^{2}\right)+\frac{h^{2}}{2} \varphi^{2}\right],
$$

and so both vanish at the global minimum $(\varphi=0$ and $\chi=v)$ as well as at a saddle point at $\varphi=\chi=0 . V_{, \chi}$ vanishes along the entire line $\chi=0$, along which the curvature of the potential is given by

$$
\left(\begin{array}{cc}
V_{, \varphi \varphi} & V_{, \varphi \chi} \\
V_{, \chi \varphi} & V_{, \chi \chi}
\end{array}\right)=\left(\begin{array}{cc}
m^{2}+3 \lambda^{2} \varphi^{2} & 0 \\
0 & \frac{1}{2} h^{2} \varphi^{2}-g^{2} v^{2}
\end{array}\right)
$$

showing that this line is a trough (local minimum in the $\chi$ direction) if $\varphi>\varphi_{\star}=\sqrt{2} g v / h(\sim v$ if $g \sim h$ ), which gets steeper and steeper the larger $\varphi$ is. Otherwise, for $\varphi<\varphi_{\star}$, the line $\chi=0$ is a

\footnotetext{
${ }^{4}$ Here an inflationary model means one having both an accelerated expansion and a mechanism for it to end, and so excludes in particular higher-dimensional configurations having only accelerated 4D expansion [30].
} 


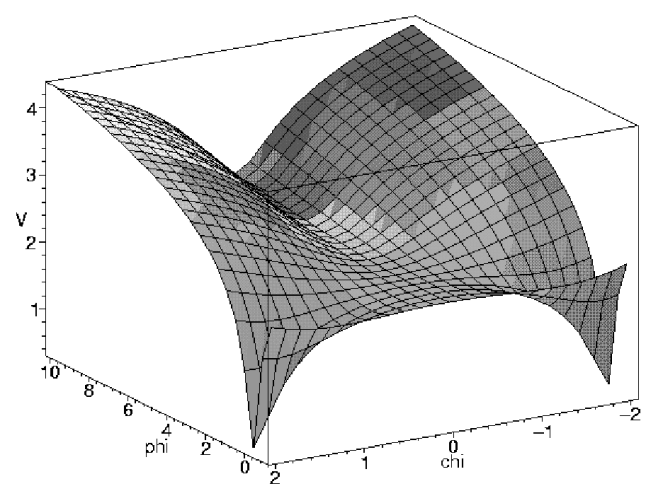

Figure 7: A sketch of the scalar potential for Hybrid Inflation.

ridge (local maximum in the $\chi$ direction), which is steepest at the saddle point at $\varphi=0$. (See Fig. 7 for a sketch of this potential.)

If $\varphi$ starts off initially much bigger than $\varphi_{\star}$, with $\chi=0$, then the potential keeps $\chi$ at zero but allows $\varphi$ to roll towards smaller values. Furthermore, if $\frac{1}{4} g^{2} v^{4} \gg \frac{1}{2} m^{2} \varphi^{2}+\frac{1}{4} \lambda^{2} \varphi^{4}$ (as is generically true for $\varphi \sim v$ if $\lambda \ll g$ and $m \ll g v)$ then $V(\varphi, \chi=0) \approx \frac{1}{4} g^{2} v^{4}$ is approximately constant during this roll. Inflation can occur provided the kinetic energy is much smaller than this constant, which the discussion of earlier sections shows occurs if the slow roll parameters describing the motion in the $\varphi$ direction,

$$
\varepsilon=\left[\frac{M_{p} \varphi\left(m^{2}+\lambda^{2} \varphi^{2}\right)}{g^{2} v^{4}}\right]^{2} \quad \text { and } \quad \eta=\frac{4 M_{p}^{2}\left(m^{2}+3 \lambda^{2} \varphi^{2}\right)}{g^{2} v^{4}}
$$

are both small. This provides an inflationary epoch, which lasts either until the slow roll parameters become too large, or until $\varphi$ falls below $\varphi_{\star}$, and so $\chi$ becomes destabilized away from zero, provoking a fast roll towards the absolute minimum at $\chi=v$. The condition $\varphi>\varphi_{\star}$ would be the first to fail if $\varepsilon$ and $\eta$ are small for $\varphi \sim v$, which is true if $m / g v$ and $\lambda / g$ are both much smaller than $v / M_{p}$.

This provides an intrinsically two-field inflationary model, where the second field can play a crucial role in bringing inflation to an end. The additional parameters available also allow a wide range for the slow roll parameters at horizon exit, and so allow examples both with $n_{s}>1$ (unlike the previous single-field models) as well as with $n_{s}<1$. For an example with $n_{s}>1$, consider the case where $\lambda \approx 0$, and $h \simeq g$, so that $\varphi_{\star} \simeq v$. Taking also $v / M_{p}=O(\delta)$ and $m / g v \sim O\left(\delta^{2}\right)$ for some $\delta \ll 1$, the number of $e$-foldings after horizon exit becomes

$$
N_{e}=\frac{1}{M_{p}} \int_{\varphi_{\star}}^{\varphi_{\mathrm{he}}} \frac{\mathrm{d} \varphi}{\sqrt{2 \varepsilon}} \simeq \frac{g^{2} v^{4}}{\sqrt{2} m^{2} M_{p}^{2}} \ln \left(\frac{\varphi_{\mathrm{he}}}{\varphi_{\star}}\right),
$$

which is $O\left(\delta^{-2}\right)$ even when $\varphi_{\text {he }}$ is also of order $v$. But for $\varphi_{\text {he }} \sim v$ we have $\eta=O\left(\delta^{2}\right) \gg \varepsilon=$ $O\left(\delta^{6}\right)>0$, which implies $n_{s}>1$ when used in eq. (2.49). 


\section{General Multi-scalar Models}

Although hybrid inflation shows that multi-field inflationary models can have interesting properties in their own right, the form of the action, eq. (3.1), is not general enough to capture the generic kinds of scalar dynamics which emerge in the low-energy limit of string theory.

The most general action describing the low-energy evolution of $N$ real scalar fields, $\phi^{a}$, is

$$
S=-\int \mathrm{d}^{4} x \sqrt{-g}\left[\frac{M_{p}^{2}}{2} R+\frac{1}{2} G_{a b}(\phi) \partial^{\mu} \phi^{a} \partial_{\mu} \phi^{b}+V(\phi)\right],
$$

where $V$ is the scalar potential, and $G_{a b}=G_{b a}$ is a positive definite symmetric matrix of functions. Notice that there is no loss in not having a function of $\phi^{a}$ in front of the Ricci curvature scalar, such as $\mathscr{L} \propto \sqrt{-g} A(\phi) R$, because any such term can be removed by performing an appropriate $\phi$-dependent Weyl re-scaling of the metric: $g_{\mu \nu} \rightarrow A^{-1}(\phi) g_{\mu \nu}$. This choice of metric which makes the Einstein-Hilbert action $\phi^{a}$-independent is called the Einstein Frame.

One way the action for Hybrid inflation, eq. (3.1), differs from eq. (3.7) is by having $G_{a b}=\delta_{a b}$, and one might ask whether this can always be arranged by performing an appropriate redefinition among the scalar fields. Although this can be done quite generally when only one scalar field is present, for more than one field it can be done (as well as ensuring $\partial_{a} G_{b c}=0$ ) only when evaluated at a specific point, $\phi^{a}=\phi_{0}^{a}$, but not simultaneously for all $\phi^{a}$. To see why this is true, notice that $G_{a b}$ transforms as a rank two tensor under field redefinitions, $\phi^{a} \rightarrow f^{a}(\phi)$ (see Exercise 9). Since $G_{a b}$ is also positive definite, it therefore has a geometrical interpretation of being a metric on the 'target' space, $M$, in which the $\phi^{a}$ take their values. As a result, we know that a change of coordinates can only ensure $G_{a b}=\delta_{a b}$ everywhere if its Riemann tensor, $R_{b c d}^{a}$, vanishes everywhere. On the other hand, the freedom to arrange $G_{a b}\left(\phi_{0}\right)=\delta_{a b}$ at any specific point $\phi_{0}^{a}$ corresponds to choosing Gaussian normal coordinates at this point.

Exercise 9: Show that under a field redefinition, $\delta \phi^{a}=\xi^{a}(\phi)$, the action of eq. (3.7) returns to the same form with $V \rightarrow V+\xi^{a} \partial_{a} V$ and $G_{a b} \rightarrow G_{a b}+\xi^{c} \partial_{c} G_{a b}+G_{a c} \partial_{b} \xi^{c}+$ $G_{c b} \partial_{a} \xi^{c}$. This shows that $V$ transforms as a scalar field, and $G_{a b}$ transforms like a rank-two tensor.

The scalar field equations for the action (3.7) are

$$
\ddot{\phi}^{a}+\Gamma_{b c}^{a}(\phi) \dot{\phi}^{b} \dot{\phi}^{c}+3 H \dot{\phi}^{a}+G^{a b} V_{, a}=0,
$$

where $V_{, a}=\partial_{a} V=\partial V / \partial \phi^{a}, G^{a b}$ is the inverse metric for $G_{a b}$ and $\Gamma_{b c}^{a}=\frac{1}{2} G^{a d}\left[\partial_{b} G_{c d}+\partial_{c} G_{b d}-\right.$ $\left.\partial_{d} G_{b c}\right]$ is the Christoffel symbol built from the target-space metric, $G_{a b}$. These are to be supplemented by the standard Friedmann (eq. (1.5)) and Raychaudhuri (eq. (1.6)) equations (or energy conservation, eq. (1.7)), where $p$ and $\rho$ are given by

$$
\rho=\frac{1}{2} G_{a b}(\phi) \dot{\phi}^{a} \dot{\phi}^{b}+V(\phi) \quad \text { and } \quad p=\frac{1}{2} G_{a b}(\phi) \dot{\phi}^{a} \dot{\phi}^{b}-V(\phi) .
$$

As before a sufficient condition for inflation is to have $V \gg \frac{1}{2} G_{a b} \dot{\phi}^{a} \dot{\phi}^{b}$ and approximately constant, and this is ensured if we may drop both the $\ddot{\phi}^{a}$ and $\Gamma_{b c}^{a} \dot{\phi}^{b} \dot{\phi}^{c}$ terms of eqs. (3.8), leading to 
the slow-roll equations, $3 H \dot{\phi}^{a}=-G^{a b} V_{, b}$. These slow-roll conditions remain good approximations for an appreciable time provided the multi-scalar generalizations of the slow-roll parameters are small over a broad enough region. As the Hybrid inflation example shows, it is important when defining these to be sure that they measure the derivatives of the potential only along the steepest direction down the potential, since this is also the direction of motion if the field starts out close to rest.

Since the gradient, $V_{, a}(\phi)$, of the scalar potential automatically points in the direction of steepest ascent for the potential, its negative naturally provides the direction down which an initiallystatic configuration starts to roll from any point, $\phi^{a}$, in the target space. Consequently, the generalization of $\varepsilon$ which measures the first derivative of the potential in this direction can be taken to be,

$$
\varepsilon=\frac{M_{p}^{2} G^{a b} V_{, a} V_{, b}}{2 V^{2}}
$$

Notice that because this transforms as a scalar under field redefinitions, it may be evaluated using any choice of fields and (unlike the formulae given earlier for single-field inflation) its use does not assume the choice $G_{a b}\left(\phi_{0}\right)=\delta_{a b}$. Furthermore, it agrees with standard multi-field definitions [7] for $\varepsilon$, since it reduces to these in normal coordinates (for which $G_{a b}\left(\phi_{0}\right)=\delta_{a b}$ ).

A multi-scalar generalization of $\eta$ is given by the smallest of the eigenvalues of the matrix of second derivatives of the potential, $V_{, a b}\left(\phi_{0}\right)$, since this defines the most unstable direction (at least in a slow-roll region where $V_{, a}$ is negligible). (Notice that if this eigenvalue is negative then we are looking for the negative eigenvalue having the largest absolute value.) In order to ensure a slow enough evolution for $\phi^{a}$ near $\phi^{a}=\phi_{0}^{a}$ it is important to evaluate this second derivative matrix only after transforming to (Gaussian normal) coordinates to ensure that $G_{a b}\left(\phi_{0}\right)=\delta_{a b}$. Alternatively, this definition can be written in a way which is equally good when evaluated using an arbitrary choice of coordinates on the target space, as follows. First define the eigenvalues, $\lambda$, of the matrix $N^{a}{ }_{b}(\phi)$, defined by

$$
N^{a}{ }_{b} e^{b}=\lambda e^{a}, \quad \text { with } \quad N^{a}{ }_{b}=\frac{M_{p}^{2} G^{a c} V_{; c b}}{V},
$$

where $V_{; c b}=V_{, c b}-\Gamma_{b c}^{a} V_{, a}$ is the covariant derivative of $V_{, b}$ using the target-space connection $\Gamma_{b c}^{a}(\phi)$. Then in an arbitrary coordinate frame $\eta=\min \lambda$, minimized over all of the possible eigenvalues of $N_{b}^{a}$. This is the appropriate generalization because as defined $\lambda$ is a scalar under scalar-field redefinitions, and because it agrees with standard definitions [7] when evaluated in the canonical Gaussian normal frame.

\section{The special case of Kähler metrics}

An important special case of the above discussion is the case which arises when the scalar fields can be grouped in to complex fields, $\left\{\phi^{a}\right\}=\left\{\phi^{i}, \bar{\phi}^{\bar{l}}\right\}$, where $\bar{\phi}^{\bar{l}}$ denotes the complex conjugate of $\phi^{i}$. In this case, if the nonzero components of the metric, $G_{a b}$, locally can be written $G_{i \bar{\jmath}}=\partial_{i} \partial_{\bar{\jmath}} K$, for some function $K=K(\phi, \bar{\phi})$, then the metric is called a Kähler metric, with $K$ being its Kähler potential. 
In this case the definition for $\varepsilon$ becomes [66]

$$
\varepsilon=\frac{M_{p}^{2} G^{i \bar{\jmath}} V_{, i} V_{, \bar{\jmath}}}{V^{2}}
$$

and $\eta$ is defined in terms of the smallest eigenvalue of the matrix

$$
\left(\begin{array}{cc}
N^{i} & N^{i} \\
N^{\bar{l}} & N^{\bar{l}}
\end{array}\right),
$$

where

$$
N_{j}^{i}=\frac{M_{p}^{2} G^{i \bar{k}} V_{\overline{, k j}}}{V} \quad \text { and } \quad N_{j}^{\bar{\imath}}=\frac{M_{p}^{2} G^{\bar{l} k}}{V}\left[V_{, k j}-G^{l \bar{m}} K_{, j k \bar{m}} V_{, l}\right]
$$

while $N_{\bar{J}}$ and $N_{\bar{J}}^{i}$ are the complex conjugates of these.

Exercise 10: Derive eqs. (3.14), by first showing that the only nonzero Christoffel symbols for a Kähler metric are $\Gamma_{j k}^{i}=G^{i \bar{m}} K_{, j k \bar{m}}$, and its complex conjugate, $\Gamma_{\bar{\jmath} \bar{k}}^{\bar{l}}$.

\section{Primordial Fluctuations}

The presence of many scalars also changes the kinds of primordial fluctuations which are possible, because with several scalars there can be perturbations, $\delta \phi^{a}$, for which the total energy density remains unchanged, $\delta \rho=0$. Any such a fluctuation is called an 'isocurvature' fluctuation, in contrast to the 'adiabatic' fluctuations involving nonzero $\delta \rho$ considered previously.

There are strong observational constraints against the existence of such isocurvature fluctuations re-entering the Hubble scale during the Hot Big Bang era. Constraints exist because isocurvature perturbations at this scale correspond to metric perturbations which emerge into the subHubble world with a zero initial amplitude, $\Phi_{i}=0$, but nonzero velocity, $\dot{\Phi}_{i} \neq 0$ (in contrast with adiabatic modes, which emerge with nonzero initial amplitude, $\Phi_{i} \neq 0$, and initially vanishing speed, $\dot{\Phi}_{i}=0$ ). This phase difference is measurable in the CMBR because it changes the value of $l$ for which the maximum peak occurs in Fig. 4. Current observations are consistent with purely adiabatic oscillations at horizon re-entry.

Multi-field inflationary models must therefore either not generate primordial isocurvature perturbations at all at horizon exit, or any such primordial perturbations must disappear sometime after horizon exit but before horizon re-entry. The absence of such fluctuations must be checked in any specific model [32].

Primordial isocurvature modes need not be a problem for an inflationary model even should they be generated at horizon exit, however, provided they are subsequently erased before horizon re-entry. This possibility exists because in the multi-field case no simple conservation law like eq. (2.40) ensures the model-independent survival of perturbed quantities. In particular, all isocurvature modes are erased if a period of thermal equilibrium occurs between Hubble exit and re-entry, because in this case all perturbations are encoded into temperature fluctuations, whose presence necessarily also perturbs the energy (and so also the gravitational potential). 


\subsection{Moduli and their Stabilization}

We now return to the main development: the description of explicit inflationary models that are grounded in stringy vacua. By restricting attention to the case $M_{c} \ll M_{s}$, the discussion can be framed within higher-dimensional field theory.

\section{D Supergravity}

The string solutions about which most is known are those which preserve some of the supersymmetries of the theory, and the higher-dimensional field theories which describe their properties below $M_{s}$ are supergravities, of which there are several in 10 dimensions. It is the bosonic fields of these supergravities that are relevant to their classical dynamics, and these always include the metric, $g_{M N}$, together with its bosonic partners under supersymmetry: a scalar dilaton, $\phi$, and a rank-2 antisymmetric gauge potential, $B_{M N}$. Other bosonic fields can also arise, depending on which supergravity is of interest. These can include gauge potentials, $A_{M}^{a}$, for 10D gauge supermultiplets (where the index ' $a$ ' runs over the generators of the relevant gauge group), as well as various kinds of $n$ th-rank skew-tensor gauge potentials, $C_{M_{1} \ldots M_{n}}$.

In addition to these 'bulk' fields, the low-energy supergravity can also include the positions, $x^{M}\left(\sigma^{\alpha}\right)$, within 10D spacetime of each of any D-branes that are allowed for the supergravity. ${ }^{5}$ Here $\sigma^{\alpha}$ are coordinates on the D-brane world sheet, with $\alpha=0,1, \ldots, p+1$ running over one time and $p$ space directions for a $\mathrm{D} p$-brane.

The action governing the dynamics of these fields comes as the sum of brane and bulk terms, $S_{10}=S_{\mathrm{br}}+S_{B}$, where the bulk action has the generic form

$$
\begin{aligned}
S_{B}=-\int \mathrm{d}^{10} x \sqrt{-g} M_{s}^{8}[ & \frac{1}{2} R+\frac{1}{2} \partial^{M} \phi \partial_{M} \phi+\frac{1}{6} e^{-\phi} H^{M N P} H_{M N P} \\
& \left.+\sum_{n} \frac{e^{c_{n} \phi}}{2(n+1) !} F_{M_{1} \ldots M_{n+1}} F^{M_{1} \ldots M_{n+1}}+\cdots\right],
\end{aligned}
$$

and $F=\mathrm{d} C$ is the exterior derivative which corresponds to the field strength appropriate to each of the skew-tensor gauge fields. (These sometimes also contain Chern-Simons terms, which in the above action are rolled into the ellipses.) The number of fields summed over, and the values of the numerical constants $c_{n}$, depend on the precise supergravity of interest. For instance, for the Type IIB supergravity of later interest the bulk action has one rank-0 potential, $C$, (i.e. a scalar), no rank-1 gauge potentials, $C_{M}$, one additional rank-2 potential, $C_{M N}$, no rank-3 potentials, $C_{M N P}$ and one rank-4 potential $C_{M N P Q}$, while the constants are $c_{0}=2, c_{2}=1$ and $c_{4}=0$.

The brane action has a similar form,

$$
S_{\mathrm{br}}=\sum_{b} T_{b} \int_{\Sigma_{b}} \mathrm{~d}^{p_{b}+1} \sigma \sqrt{-\gamma} e^{\lambda_{b} \phi}(1+\cdots)+\mu_{b} \int_{\Sigma_{b}}\left(\Omega_{b}+\cdots\right)
$$

where the sum is over the branes present, and the integral is over the $(p+1)$-dimensional worldvolume of each $\mathrm{D} p$-brane. Here $\lambda_{b}$ is a known constant, equal to $\left(p_{b}-3\right) / 4$ for 10D supergravity,

\footnotetext{
${ }^{5}$ In principle, Type IIA supergravity allows D0, D2, D4, D6 and D8 branes, while Type IIB supergravity allows D1, D3, D5, D7 and D9 branes. No D-branes arise at all in heterotic vacua. 5+1 dimensional surfaces called NS5-branes can also exist for each of these supergravities.
} 
and the form $\Omega_{b}$ appearing in the second integral is either the particular potential, $C_{M_{1} \ldots M_{p+1}}$, whose rank is $p+1$, or the Hodge dual (obtained by contracting one of the $C$ 's with the 10D Levi-Civita tensor, $\varepsilon_{M_{1} \ldots M_{10}}$ ) of a form of rank $9-p$. One such a form exists for each kind of brane allowed by each of the possible supergravities. The dimensionful constants $T_{b}$ and $\mu_{b}$ in these expressions are proportional to $M_{s}^{p+1}$ (with known numerical coefficients). $T_{p}$ has the physical interpretation of the brane tension, or energy per unit world-volume. Finally, the world sheet 'metric' appearing in eq. (3.16) is given by

$$
\gamma_{\alpha \beta}(\sigma)=\partial_{\alpha} x^{M} \partial_{\beta} x^{N}\left[g_{M N}+B_{M N}+\frac{1}{M_{s}^{2}} F_{M N}\right],
$$

where $F_{M N}=\partial_{M} A_{N}-\partial_{N} A_{M}$ is the $U(1)$ gauge field associated with those open strings both of whose ends terminate on the brane in question. (A more complicated expression holds when $N$ branes sit at the same point in spacetime, since this promotes the gauge group to $U(N)$.)

\section{Moduli}

Of particular interest are those string vacua for which only the 4 dimensions of everyday experience are noncompact, and the other 6 dimensions are compactified with a size corresponding to an energy scale $M_{c}$. For $M_{c} \ll M_{s}$ these correspond to semiclassical solutions to the corresponding $10 \mathrm{D}$ supergravity equations. A considerable amount is known about these solutions in the case that the compactification preserves at least one supersymmetry in 4D.

In the absence of branes the supersymmetric solutions have a metric of the product form [33]

$$
\mathrm{d} s^{2}=\eta_{\mu v} \mathrm{~d} x^{\mu} \mathrm{d} x^{v}+g_{m n}(y) \mathrm{d} y^{m} \mathrm{~d} y^{n},
$$

where $x^{\mu}$ are coordinates for the noncompact 4 dimensions, $y^{m}$ label the compact 6 dimensions and $\eta_{\mu \nu}$ is the usual 4D Minkowski metric. Among other things, $N=1$ supersymmetry in 4D requires the extra-dimensional metric, $g_{m n}$, to be Calabi-Yau (i.e. Ricci-flat geometries having $S U(3)$ holonomy). There is generically a many-parameter family of such metrics which all share the same (fairly complicated) topology, $g_{m n}(y)=g_{m n}(y ; \omega)$, where $\omega_{a}$ represent the parameters required to fully describe the geometry.

The parameters required to describe a geometry are known as moduli, and generically arise when solving the Einstein equations. A simple example of a geometry having moduli is given by the 2-dimensional torus, which is defined by the condition that its Riemann curvature vanishes: $R^{m}{ }_{n p q}=0$ in a 2D space with boundary conditions $y^{1} \simeq y^{1}+1$ and $y^{2} \simeq y^{2}+1$. The general $2 \mathrm{D}$ metric which solves this equation is

$$
\mathrm{d} s^{2}=a\left[\left(\mathrm{~d} y^{1}\right)^{2}+2 b \mathrm{~d} y^{1} \mathrm{~d} y^{2}+c\left(\mathrm{~d} y^{2}\right)^{2}\right],
$$

where $a, b$ and $c$ are arbitrary constants, and so are the three moduli of a 2-torus. One of these, $a$, describes overall re-scalings of the size of the metric (the so-called breathing mode), and is generically a modulus because of a scale invariance of the supergravity equations in higher dimensions. The other two moduli describe changes to the geometry at fixed volume (specifically changes to 
what is called its complex structure). A Calabi-Yau geometry can have hundreds of similar moduli, which can be divided into two categories: those describing modifications to its complex structure, and the rest - including the breathing mode - that are known as Kähler moduli.

Moduli are of particular interest when studying compactifications because the classical field equations guarantee the existence of a massless 4D scalar field for each modulus of the extradimensional metric. To see how this works, first recall how to compactify a fluctuation in a 10D scalar field, $\delta \phi(x, y)$, whose 10D field equation is $\square_{10} \delta \phi=g^{M N} D_{M} D_{N} \delta \phi=0$. Evaluated for a product metric like eq. (3.18), this becomes $\left(\square_{4}+\square_{6}\right) \delta \phi=0$, where $\square_{6}=g^{m n} D_{m} D_{n}$ and $\square_{4}=$ $\eta^{\mu v} \partial_{\mu} \partial_{v}$. If we decompose $\delta \phi(x, y)$ in terms of eigenfunctions, $u_{k}(y)$, of $\square_{6}-i . e$. where $\square_{6} u_{k}=$ $-\mu_{k}^{2} u_{k}$ - we have

$$
\delta \phi(x, y)=\sum_{k} \varphi_{k}(x) u_{k}(y),
$$

and the equations of motion for $\phi$ imply $\left(\square_{4}-\mu_{k}^{2}\right) \varphi_{k}=0$. The 10D field decomposes as an infinite number of 4D Kaluza-Klein fields, each of whose 4D mass is given by the corresponding eigenvalue, $\mu_{k}$. In particular a massless mode in 4D corresponds to a zero eigenvalue: $\square_{6} u_{k}=0$.

A similar analysis also applies for the fluctuations, $\delta g_{M N}(x, y)$, in the $10 \mathrm{D}$ metric about a specific background geometry such as eq. (3.18). Focussing on metric components in the extra dimensions, $\delta g_{m n}(x, y)$, allows an expansion similar to eq. (3.20)

$$
\delta g_{m n}(x, y)=\sum_{k} \varphi_{k}(x) h_{m n}^{k}(y),
$$

where $h_{m n}(y)$ are tensor eigenfunctions for a particular 6D differential operator (the Lichnerowitz operator) obtained by linearizing the Einstein equations, $\Delta_{6} h_{m n}^{k}=-\mu_{k}^{2} h_{m n}^{k}$. Again the 10D equation of motion, $\Delta_{10} \delta g_{m n}=0$, implies each 4D mode, $\varphi_{k}(x)$, satisfies $\left(\square_{4}-\mu_{k}^{2}\right) \varphi_{k}=0$, and so has mass $\mu_{k}$.

The significance of moduli is that they provide zero eigenfunctions for $\Delta_{6}$, and so identify massless 4D scalar fields within the KK reduction of the extra-dimensional metric. The zero eigenfunction is given by the variation of the background metric in the direction of the moduli. Schematically, if $\omega_{a}$ are the moduli of the background metric, $g_{m n}(y ; \omega)$, and if $h_{m n}^{a}=\partial g_{m n} / \partial \omega_{a}$, then $\Delta_{6} h_{m n}^{a}=0$. Physically, these are zero eigenfunctions because varying a modulus in a given solution to the Einstein equations gives (by definition) a new solution to the same equations, and so in particular an infinitesimal variation in this direction is a zero mode of the linearized equations.

Because the 4D moduli fields, $\varphi_{a}(x)$, are massless they necessarily appear in the low-energy $4 \mathrm{D}$ effective action which governs the dynamics at scales below the KK scale, $M_{c}$. If we focus purely on the moduli and the 4D metric (and ignore other fields), then the low-energy part of this action must take the general form of eq. (3.7), but with a potential, $V$, which is independent of the moduli, $\varphi^{a}(x)$.

\section{Moduli and inflation}

Moduli (and any other classically massless scalars) are a mixed blessing for inflationary models. The Good News is they provide a large number of candidate scalar fields in the $4 \mathrm{D}$ effective 
theory, any of which might play the role of the inflaton. Furthermore, a slow roll could be possible because their potential is often very shallow, being required to be flat to the accuracy with which it is known that configurations like $g_{m n}(y ; \omega)$ are solutions for all $\omega_{a}$. Typically the field in question is only approximately a modulus, although some can be exactly massless if one of the supersymmetries is unbroken. Even in supersymmetric cases it often happens that moduli remain massless to all orders in perturbation theory, but appear in the 4D scalar potential once non-perturbative effects are considered.

Indeed a number of these scalar fields have been proposed as possible inflatons [34], however before the discovery of branes within string theory all of the proposed inflationary scenarios had difficulties. One difficulty for the moduli of supersymmetric vacua was the need to compute nonperturbative contributions, which made the calculation of the inflaton potential difficult. Branes provide a way forward on two fronts: they allow supersymmetry-breaking effects to be more simply computed, such as with the use of brane-antibrane dynamics; and they play a central role in the geometries arising in the modulus-stabilization programme. The ability to compute explicitly led to an explosion of inflaton proposals, including metric moduli [35], massless modes arising from extra-dimensional gauge fields [36], inter-brane separations [23, 24, 25, 37, 38], more stringy modes, [39] and so on.

On the other hand, the Bad News is that it is usually impossible to know for sure whether a given light scalar can be the inflaton until the full potential is understood which governs the dynamics of all of the low-energy moduli. This is because a slow roll requires the potential to be shallow in its steepest downward direction. If one finds an inflaton potential that is shallow enough to obtain inflation before understanding the corrections which stabilize some of the moduli, one must worry that these corrections ruin the inflationary solution by providing steeper directions along which the inflaton could roll without inflating. Unfortunately, progress on understanding modulus stabilization was a long time coming in string theory, and the lack of this understanding proved to be a long-standing obstacle to identifying how inflation might arise within a stringy context.

\section{Modulus Stabilization: Branes and Fluxes}

Major progress on string inflation became possible with the development of tools for understanding how to stabilize most of the moduli for a few kinds of stringy vacua. This progress started with the identification of how to generalize [40] the 4D supersymmetric compactifications of the field equations of Type IIB supergravity in 10 dimensions to include the presence of parallel D3, D7 branes (plus 7+1-dimensional surfaces having negative tension, called orientifold planes).

These branes complicate the dynamics of the internal dimensions in several important ways. First, they do so through the gravitational fields they create, which have the effect of modifying the metric of eq. (3.18) into the following form,

$$
\mathrm{d} s^{2}=h^{-1 / 2}(y) \eta_{\mu v} \mathrm{~d} x^{\mu} \mathrm{d} x^{v}+h^{1 / 2}(y) g_{m n}(y) \mathrm{d} y^{m} \mathrm{~d} y^{n}
$$

with the warp factor, $h(y)$, depending on the positions of the various branes. The metric $g_{m n}(y)$ 
appearing here is a Ricci-flat Calabi-Yau type metric, of the form which arose in the absence of the branes.

A second important difference to the dynamics of the internal dimensions which arises once branes are present is the presence of nontrivial configurations of the various antisymmetric tensor fields, for which they act as sources. The total flux of these fields through topologically nontrivial surfaces in the extra dimensions is quantized, such as

$$
M_{s}^{2} \int_{S} F \propto n_{1} \quad \text { and } \quad M_{s}^{2} \int_{S} H \propto n_{2},
$$

where $S$ is a 3-cycle, $F=\mathrm{d} C$ and $H=\mathrm{d} B$ are 3 -form fluxes, and $n_{1}$ and $n_{2}$ are integers that depend on which 3-surface $S$ is considered. The presence of such fluxes has two important consequences: (i) they can (but need not) break the remaining 4D supersymmetry, and (ii) they can remove some of the moduli of the extra-dimensional geometry, such as changes to the area of these surfaces $S$. These are no longer moduli when fluxes are present because flux quantization implies the value of fields like $C_{M N}$ must grow as the areas of these surfaces shrink, ensuring such changes come with an energy cost.

A third potential contribution of branes to extra-dimensional dynamics is the tension of the branes themselves. In particular, since D7 branes fill 7 spatial dimensions, and only 3 of these are the noncompact ones we see, they must also extend into 4 of the compact 6 dimensions. Typically they do so by 'wrapping' themselves around a non-contractable surface, or 4-cycle, in these extra dimensions. But D7 branes have a fixed tension, $T_{7} \propto M_{s}^{8}$, and so such wrappings provide an energy cost for increasing the moduli describing the volume of the cycles about which branes wrap. Precisely what this energy cost is depends on the relative number of different kinds of branes (positive tension $D 7$ branes, or negative tension orientifold $O 7$ and $O 3$ planes) wrapping any given cycle, a number which is itself subject to the topological constraint ('tadpole condition') that the net $D 3$ and $D 7$ charges must vanish (much in the same way that Gauss' Law requires the net electric charge in any compact volume to vanish).

In the end one expects such geometries having both branes and fluxes to have fewer moduli than do those without branes and fluxes, and this is indeed what is found. In particular, for the supersymmetric Type IIB compactifications described here, the fluxes and branes turn out to remove all of the complex structure moduli that are associated with the Calabi-Yau metric, $g_{m n}$, appearing in eq. (3.22). But not all of the moduli of $g_{m n}$ are lifted in this way, with the Kähler moduli (including the breathing mode) remaining at the classical level, even in the presence of branes and fluxes.

\section{Warped Throats}

The extra dimensions which result in this way can have a complicated and rich geometry, including the possibility of warped throats along which the warp factor, $h(y)$, varies strongly. The $6 \mathrm{D}$ geometry in such a throat is well approximated by the following polar-coordinate-like form

$$
\mathrm{d} s^{2}=h^{-1 / 2} \eta_{\mu v} \mathrm{~d} x^{\mu} \mathrm{d} x^{v}+h^{1 / 2}\left[\mathrm{~d} \rho^{2}+\rho^{2} \mathrm{~d} s_{5}^{2}\right] \quad \text { with } \quad h \simeq a^{4}+b^{4} / \rho^{4},
$$




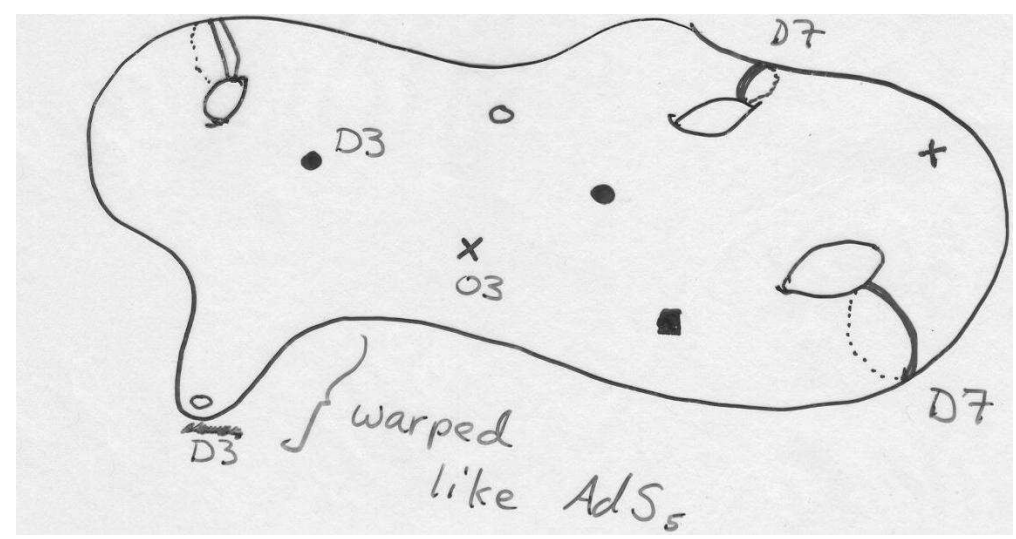

Figure 8: A cartoon of a Type IIB extra-dimensional configuration.

where $\rho$ denotes proper distance along the throat (measured with the metric $g_{m n}$ ) and $\mathrm{d} s_{5}^{2}$ is a known metric describing the 5 other 'angular' directions. These approximations work well away from the throat's 'base' (i.e. $\rho \gg b / a$, where $h$ becomes more slowly varying and joins into the bulk of the internal dimensions). They also apply not too close to its 'tip' ( $\rho \rightarrow 0$, where the conical singularity generically present in the metric, $g_{m n}$, becomes smoothed out).

Notice that for $\rho \ll b / a$ we have $h \propto \rho^{-4}$ and so the metric, eq. (3.24), takes the approximate form

$$
\begin{aligned}
\mathrm{d} s^{2} & \simeq \frac{\rho^{2}}{b^{2}} \eta_{\mu v} \mathrm{~d} x^{\mu} \mathrm{d} x^{v}+\frac{b^{2} \mathrm{~d} \rho^{2}}{\rho^{2}}+\mathrm{d} s_{5}^{2} \\
& =e^{2 \xi / b} \eta_{\mu v} \mathrm{~d} x^{\mu} \mathrm{d} x^{\nu}+\mathrm{d} \xi^{2}+\mathrm{d} s_{5}^{2}
\end{aligned}
$$

where we change variables using $\rho=\rho_{0} e^{\xi / b}$, and absorb a factor of $\rho_{0}$ into the 4D coordinates, $x^{\mu}$. The restriction of this metric to the 5 dimensions spanned by the coordinates $\left\{x^{\mu}, \xi\right\}$ is the 5D de Sitter metric, and so eq. (3.25) shows that the 4D warp factor varies exponentially quickly with proper distance, $\xi$, along the throat. (Once corrections to the geometry near the throat's tip are included one finds $h_{\text {tip }}=h(\rho \rightarrow 0)$ does not diverge. $)$ This is precisely the kind of fast variation of $4 \mathrm{D}$ scale in the extra dimensions which could play a role in the hierarchy problem, à la Randall and Sundrum [41].

\section{The 4D Point of View}

The Type IIB compactifications to 4 dimensions of ref. [40] generically all share two properties: $(i)$ they are either $N=1$ supersymmetric in $4 \mathrm{D}$, or break this supersymmetry by a small amount compared to $M_{c}$; and (ii) they preserve at least one (but usually many) massless moduli at the classical level. Consequently they can have an interesting dynamics at energies well below $M_{c}$, which it should be possible to capture with an $N=1$ supersymmetric $4 \mathrm{D}$ effective field theory.

The field content of any such a 4D supergravity generically consists of: ( $i$ ) chiral matter multiplets, whose bosonic components are complex scalar fields, $\varphi^{i}$; (ii) gauge multiplets, whose 
bosonic components are gauge potentials, $A_{\mu}^{a}$; and (iii) the supergravity multiplet, whose bosonic component is the massless KK mode of the 4D metric itself, $g_{\mu \nu}$. (If more than one 4D supersymmetry were to survive to energies below $M_{c}$ then a fourth kind of multiplet, consisting of a gravitino and a gauge boson, would also be required.) Since the surviving moduli are 4D scalars, we expect these to fall into 4D chiral multiplets, and so be represented by complex scalar fields, $\varphi^{i}$.

Once expressed in the 4D Einstein frame (i.e. with the metric chosen so that the 4D gravity lagrangian density is $\mathscr{L}=-\frac{1}{2} M_{p}^{2} \sqrt{-g} g^{\mu v} R_{\mu v}$ ) the interactions amongst these fields are described by 4D $N=1$ supergravity [42], which (at low energies, where the lowest derivatives dominate) is completely characterized by three functions of the chiral scalars: $(i)$ the holomorphic superpotential, $W(\varphi)$; (ii) the holomorphic gauge coupling function, $f_{a b}(\varphi)$; and (iii) the Kähler potential, $K(\varphi, \bar{\varphi})$. In particular, the kinetic terms for the gauge potentials, $A_{\mu}^{a}$, are given in terms of $f_{a b}$ by

$$
\frac{\mathscr{L}_{g k i n}}{\sqrt{-g}}=-\frac{1}{4}\left(\operatorname{Re} f_{a b}\right) F_{\mu v}^{a} F_{a}^{\mu v}
$$

and so if $f_{a b}=f_{a} \boldsymbol{\delta}_{a b}$ then the gauge coupling is given by $1 / g_{a}^{2}=\operatorname{Re} f_{a}$. The scalar-field kinetic terms and self-interactions are similarly given by

$$
\frac{\mathscr{L}_{s}}{\sqrt{-g}}=-G_{i \bar{\jmath}}(\varphi, \bar{\varphi}) \partial^{\mu} \varphi^{i} \partial_{\mu} \bar{\varphi}^{\bar{\jmath}}-V(\varphi, \bar{\varphi})
$$

with target space metric for the scalars given by $G_{i \bar{j}}=K_{, i \bar{j}}$, which is a Kähler metric, and we adopt Planck units for which $M_{p}=(8 \pi G)^{-1 / 2}=1$.

The scalar potential is $V=V_{F}+V_{D}$, where

$$
V_{D}=\frac{1}{2} f^{a b} D_{a} D_{b} \quad \text { with } \quad D_{a}=K_{, i} \delta_{a} \varphi^{i}
$$

$f^{a b}$ is the matrix inverse of the gauge coupling matrix, $\operatorname{Re} f_{a b}$, and $\delta_{a} \varphi^{i}$ denotes the variation of the scalar fields under a gauge transformation (so $V_{D}$ arises only when there are low-energy gauge multiplets present, coupled to the scalars). The remaining term in $V$ is

$$
V_{F}=e^{K}\left[G^{\bar{j} j} \overline{D_{i} W} D_{j} W-3|W|^{2}\right],
$$

where, as usual, $G^{\bar{j} j}$ is the inverse metric to $G_{i \bar{j}}$, and the quantity $D_{i} W$ denotes the Kähler covariant derivative of $W$, defined by

$$
D_{i} W=W_{, i}+K_{, i} W
$$

It turns out that $D_{i} W$ is the order parameter for supersymmetry breaking, and so must vanish for stationary points of this potential to preserve supersymmetry.

Exercise 11: Show that any solution to $D_{i} W=0$ (for all $i$ ) is also a stationary point for $V_{F}$. Show also that gauge invariance of the superpotential, $W_{, i} \delta_{a} \varphi^{i}=0$, ensures that $D_{i} W=0$ implies $V_{D}=0$. 
The functions $K$ and $W$ can be computed semiclassically for the remaining moduli in the Type IIB compactifications of ref. [40] by directly dimensionally reducing the higher-dimensional action, and this gives

$$
K=-2 \ln \left(M_{s}^{6} \mathscr{V}_{6}\right) \quad \text { and } \quad W=W_{0},
$$

where $\mathscr{V}_{6}$ denotes the volume of the internal 6 dimensions, as measured using the metric $g_{m n}$ and expressed as a function of its complex moduli, $\varphi^{i}$. $W_{0}$, on the other hand, is a $\varphi^{i}$-independent constant, which can be computed in terms of the extra-dimensional fluxes which have been turned on [43]. If the fluxes involved do not break supersymmetry, then $W_{0}$ vanishes, but $W_{0}$ is typically nonzero if these fluxes break the remaining 4D supersymmetry.

Example with one modulus: For example, one modulus which always survives at the classical level (due to a classical scale invariance of the higher-dimensional supergravity equations) is the field corresponding to the overall breathing mode of the extra dimensions. Writing the internal metric as $g_{m n}(y)=r^{2} \hat{g}_{m n}(y)$, with, say, $M_{s}^{6} \int \mathrm{d}^{6} y \sqrt{\hat{g}}=1$, then we first seek the complex field, $\varphi$, which contains the 4D modulus $r(x)$. In principle this can be obtained by examining the supersymmetry transformation laws, to see which fields transform in the standard form for a 4D multiplet [44], but a shortcut to the result can be found by examining the dependence on $r$ of the gauge kinetic terms for a gauge field on one of the D7 branes wrapped about some 4-cycle $\Sigma$. The result obtained by dimensional reduction is

$$
\begin{aligned}
\mathscr{L}_{g k i n} & =-\frac{1}{4} \int_{\Sigma} \mathrm{d}^{4} y \sqrt{-g} g^{\mu v} g^{\lambda \rho} F_{\mu \lambda} F_{v \rho}+\cdots \\
& =-\frac{r^{4}}{4} \eta^{\mu v} \eta^{\lambda \rho} F_{\mu \lambda} F_{v \rho} \int \mathrm{d}^{4} y \sqrt{-\hat{g}} h(y)+\cdots,
\end{aligned}
$$

which, when compared with the supersymmetric 4D gauge kinetic function shows that $\operatorname{Re} f=k r^{4}$, with $k \propto \int_{\Sigma} \mathrm{d}^{4} y \sqrt{\hat{g}} h$. Since 4D supersymmetry requires $f$ to be a holomorphic function of the complex modulus $\varphi$, it follows that we can define $\varphi$ such that $f=\varphi$, with $\operatorname{Re} \varphi=k r^{4}$.

Given this relation between $\varphi$ and $r$ we may compute the Kähler potential $K$, using the known $r$-dependence of the 6D volume: $M_{s}^{6} \mathscr{V}_{6}=M_{s}^{6} r^{6} \int \mathrm{d}^{6} y \sqrt{\hat{g}}=r^{6}$. This shows that $\mathscr{V}_{6} \propto(\operatorname{Re} \varphi)^{3 / 2}$, and so

$$
K(\varphi, \bar{\varphi})=-2 \ln \left(M_{s}^{6} \mathscr{V}_{6}\right)=-3 \ln (\varphi+\bar{\varphi}),
$$

up to an irrelevant additive constant. The fact that $K$ depends only on $\operatorname{Re} \varphi$ can also be deduced on symmetry grounds once the supersymmetry transformations are used to identify which fields appear in $\operatorname{Im} \varphi . K$ cannot depend on $\operatorname{Im} \varphi$ at the classical level because the theory turns out to be invariant under constant shifts of $\operatorname{Im} \varphi$.

Exercise 12: Verify that using the Kähler potential of eq. (3.33) in eq. (3.27) gives the correct kinetic terms for $r(x)$, by comparing the result with what you obtain by directly dimensionally reducing the higher-dimensional Einstein-Hilbert action, $\mathscr{L}=$ $-\frac{1}{2} M_{s}^{8} \sqrt{-g} R$, using the metric, eq. (3.22), with $g_{m n}=r^{2}(x) \hat{g}_{m n}(y)$. Do not forget to go to the 4D Einstein frame by also re-scaling the 4D metric, $g_{\mu \nu} \rightarrow r^{-6} g_{\mu \nu}$. 
A check on the whole picture comes when the above results for $W$ and $K$ are used to compute the scalar potential for $\varphi$, using the general expression, eq. (3.29). Consistency requires the result must vanish, $V=0$, since $\varphi$ is a modulus and so cannot have a scalar potential (to the accuracy used to derive $W$ and $K$ ). Notice first that $\varphi$ does not transform under gauge transformations (so long as none of the D7 gauge groups are anomalous), so $V_{D}=0$ and $V=V_{F}$. Specializing eq. (3.29) for $V_{F}$ to a constant superpotential, $W=W_{0}$, then gives

$$
V=e^{K}\left[G^{\bar{l} j} K_{, \bar{l}} K_{, j}-3\right]\left|W_{0}\right|^{2} .
$$

Finally, using eq. (3.33) in this expression gives $V_{F} \equiv 0$ for all $\varphi$, because the Kähler potential satisfies the remarkable identity

$$
G^{\bar{l} j} K_{, \bar{l}} K_{, j} \equiv 3 .
$$

Models whose Kähler potential satisfies this identity are known as no-scale models [42, 45]. They play an important role in low-energy string theory because they capture the property that the lowenergy 4D potential cannot depend on moduli fields.

Since $V$ vanishes, any value of $\varphi$ provides an equally good classical vacuum for the low-energy $4 \mathrm{D}$ theory. Notice, however, that if $W_{0} \neq 0$ then supersymmetry is typically broken for most of these values, since the order parameter for supersymmetry breaking is $D_{\varphi} W=K_{, \varphi} W_{0}$. This ensures the

effective $4 \mathrm{D}$ picture agrees with the higher-dimensional point of view, because $W_{0}$ is only nonzero if the higher-dimensional fluxes break 4D supersymmetry.

Examples with several moduli: A second example of practical later interest is to compactifications for which more than one modulus survives at the classical level, corresponding to a collection of complex moduli, $\varphi^{i}$. For many of these the Kähler potential, $K$, of the moduli has been explicitly computed, with some having the form

$$
K(\varphi, \bar{\varphi})=-2 \ln \left[\left(\tau^{1}\right)^{3 / 2}-\sum_{i \neq 1} k_{i}\left(\tau^{i}\right)^{3 / 2}\right],
$$

where $\tau^{i}=\operatorname{Re} \varphi^{i}$ and $k_{i}$ are calculable constants for a given Calabi-Yau geometry. In these models $V=V_{F}$, and the superpotential is constant, $W=W_{0}$, so we are again led to eq. (3.34) as the scalar potential. Remarkably, we again obtain $V_{F} \equiv 0$ in this case, because the Kähler potential, eq. (3.36), also satisfies the no-scale identity $G^{\bar{\imath} j} K_{, \bar{l}} K_{, j} \equiv 3$.

Exercise 13: Explicitly show that the Kähler potential given in eq. (3.36) satisfies the no-scale identity, eq. (3.35).

\section{Corrections to the Semi-classical Picture}

A consistent low-energy 4D picture for the dynamics of moduli exists for Type IIB string vacua, but so far the resulting scalar dynamics does not inflate because the scalar potentials are precisely flat. However the functions $K$ and $W$ used to this point are computed by direct dimensional reduction using the higher-dimensional classical action, and the potential can become more 
complicated once corrections are included which introduce an energy cost to changing the value of the low-energy fields, $\varphi^{i}$.

There are two important kinds of corrections of this sort which are known to arise: $(i)$ string loop corrections, involving powers of $g_{s} \sim e^{\phi}$; and (ii) $\alpha^{\prime}$ corrections, to do with the higherdimensional supergravity equations themselves only being low-energy approximations to the full string theory. (The notation $\alpha^{\prime} \propto M_{s}^{-2}$ is defined for historical reasons, and controls the second type of corrections because they are typically suppressed by powers of a low-energy scale (like $M_{c}$ ) compared with $1 / M_{s}^{2}=\alpha^{\prime}$.)

Some of the effects of these corrections on $K, W$ and $f_{a b}$ are known. It is known that the holomorphic superpotential, $W$, does not receive either of these kinds of corrections, to all orders in perturbation theory, a result called the non-renormalization theorem [46]. It can, however, be corrected once non-perturbative contributions are included. The Kähler potential, $K$, is not similarly protected, however, with the contribution of higher-curvature $\alpha^{\prime}$ corrections in the extradimensional action correcting $K$ to become [47]

$$
K=-2 \ln \left(M_{s}^{6} \mathscr{V}_{6}+\frac{\xi}{2}\right)
$$

where $\xi=-\chi(\mathscr{M}) /\left[2(2 \pi)^{3}\right]$ being a calculable coefficient depending on the Euler number, $\chi(\mathscr{M})$, of the extra-dimensional geometry, $\mathscr{M}$. Notice that the new term inside the logarithm is suppressed relative to the first one by powers of $1 / \mathscr{V}_{6}$, as is typical for $\alpha^{\prime}$ corrections. Notice also that the corrected Kähler potential no longer satisfies the no-scale identity, eq. (3.35).

\section{The KKLT Framework}

The first approach to fix all of the moduli within the Type IIB framework - by Kachru, Kallosh, Linde and Trivedi, or KKLT [48] - starts with the assumption that only one modulus, $\varphi$, survives the flux compactification, leading to a constant superpotential, $W=W_{0}$, and the Kähler potential of eq. (3.33). The remaining modulus is then imagined to be fixed through a non-perturbative correction to the superpotential, of the form

$$
W(\varphi)=W_{0}+A \exp [-a \varphi]
$$

where $A$ and $a$ are both constants. This functional form for the non-perturbative correction to $W$ is known to arise in two kinds of situations: in the presence of some brane-related instantons [49], or if the low-energy gauge group associated with some of the D7 branes contains an asymptoticallyfree non-abelian gauge group, $G$. (For instance, since the gauge coupling function is $f_{a b}(\varphi)=\varphi \delta_{a b}$ for such a gauge group, if $G=S U(N)$ and there are no matter multiplets carrying $S U(N)$ quantum numbers, then condensation of gauginos $[50,51]$ in the vacuum leads to a superpotential of the above form, with $A$ nonzero and $a=2 \pi / N$. In this case the exponential dependence of $W$ on $\varphi$ reflects a vacuum energy which depends non-perturbatively on the gauge coupling constant, $g^{-2} \propto \operatorname{Re} \varphi$.)

KKLT analyze the potential generated using the non-perturbative superpotential of eq. (3.38) together with the uncorrected Kähler potential of eq. (3.33). Is it consistent to use non-perturbative 
corrections to $W$ when not keeping perturbative contributions to $K$ ? It can be, depending on the size of $W_{0}$. To see this imagine that $K=K_{0}+K_{p}$ and $W=W_{0}+W_{n p}$, where $K_{p}$ denotes the perturbative corrections to $K$ and $W_{n p}$ is the (much smaller) non-perturbative contribution to $W$. The corresponding contributions to $V_{F}$ then have the schematic form $V_{F}=V_{0}+V_{p}+V_{n p}$ where $V_{0}=0$ because of the no-scale form of the Kähler potential, while

$$
\begin{aligned}
V_{p} & =O\left(K_{p}\left|W_{0}\right|^{2}\right)+O\left(K_{p}^{2}\left|W_{0}\right|^{2}\right)+\cdots \\
V_{n p} & =O\left(W_{0} W_{n p}\right)+O\left(K_{p} W_{0} W_{n p}\right)+O\left(\left|W_{n p}\right|^{2}\right)+\cdots,
\end{aligned}
$$

and the ellipses contain further subdominant terms. For generic values of $W_{0}$ the perturbative contributions to $V_{F}$ dominate the non-perturbative ones, but if $W_{0}$ should be anomalously small, e.g. $W_{0} \sim W_{n p}$, then the terms involving $K_{p}$ become subdominant even when $W_{n p}$ cannot be neglected.

Using the leading-order Kähler potential, eq. (3.33), and including the non-perturbative superpotential, eq. (3.38), gives a potential which depends nontrivially on $\varphi$, with $V \rightarrow 0$ as $|\varphi| \rightarrow \infty$, falling to a nontrivial minimum for nonzero $\varphi=\varphi_{m}$ [48]. Furthermore, although the domain of validity of the $\alpha^{\prime}$ expansion is large $\operatorname{Re} \varphi$, this domain can extend down to small enough values to trust the position of this minimum provided we choose $W_{0} \sim W_{n p}\left(\varphi_{m}\right)$.

The resulting minimum turns out to be supersymmetric, since

$$
\left.D_{\varphi} W\right|_{\varphi_{m}}=-a A e^{-a \varphi_{m}}-\frac{3\left[W_{0}+A e^{-a \varphi_{m}}\right]}{\varphi_{m}+\bar{\varphi}_{m}}=0
$$

there, and so

$$
V\left(\varphi_{m}, \bar{\varphi}_{m}\right)=-\frac{3\left|W_{0}+A e^{-a \varphi_{m}}\right|^{2}}{\left(\varphi_{m}+\bar{\varphi}_{m}\right)^{3}}=-\frac{\left|a A e^{-a \varphi_{m}}\right|^{2}}{3\left(\varphi_{m}+\bar{\varphi}_{m}\right)}<0 .
$$

\section{Uplifting}

Although this successfully fixes the last of the moduli, it does so in a way which does not break supersymmetry, and with the geometry of the noncompact 4 dimensions being given by antide Sitter space due to the negative vacuum energy density, eq. (3.41). For this reason it is useful to modify the system slightly, both to break supersymmetry and to raise the vacuum energy to zero (or positive) values. The idea is to do so in a way which does not ruin the success of the modulus stabilization just discussed.

KKLT suggested doing so by adding an anti-D3 brane to the system. The problem is that such a $\overline{\mathrm{D} 3}$ breaks all of the supersymmetries that are preserved by the Calabi-Yau geometry, and so need not appear within the effective 4D theory in a way that is captured by 4D $N=1$ supergravity. Although this gives much less control over the corrections to the calculation, the damage can be kept small if the contribution of the antibrane to the low-energy action can be made parametrically weak. This can plausibly be done in the case that there is a strongly warped throat, because in this case the antibrane can minimize its energy by moving to the throat's tip. It can do so because at the tip the dimensional reduction of the anti-brane tension (starting in the 10D Einstein frame) is small, with

$$
\mathscr{L}_{\overline{D 3}}=-T_{3} \int \mathrm{d}^{4} x \sqrt{-g}=-T_{3} \int \mathrm{d}^{4} x \frac{\sqrt{-\hat{g}}}{h_{\text {tip }} r^{12}}=-k^{3} T_{3} \int \mathrm{d}^{4} x \frac{\sqrt{-\hat{g}}}{h_{\text {tip }}(\operatorname{Re} \varphi)^{3}} .
$$


Here the second equality uses $g_{\mu \nu}=r^{-6} \hat{g}_{\mu \nu}$, as is required to go to the 4D Einstein frame once we re-scale the internal metric by $g_{m n}=r^{2} \hat{g}_{m n}$, and the third equality uses the connection $\operatorname{Re} \varphi=k r^{4}$. Since the value of the warp factor at the throat's tip turns out to depend on $r$ like $h_{\text {tip }}=h_{0} r^{-4}=$ $k h_{0}(\operatorname{Re} \varphi)^{-1}$, we see that the antibrane contribution to the potential becomes

$$
V_{\overline{D 3}}=\frac{\mathscr{E}}{(\operatorname{Re} \varphi)^{2}},
$$

where $\mathscr{E} \simeq k^{2} T_{3} / h_{0}>0$.

The point of this exercise is that the value of the parameter, $h_{0}$, can be tuned over an extremely wide range of values because it is given in Type IIB compactifications as an exponential of the various integers which label the quantized fluxes within the extra dimensions. Consequently, it is possible to adjust these integers to ensure that $h_{0}$ is sufficiently large that the contribution of the antibrane to the low-energy action can be computed perturbatively in $\mathscr{E}$, which to leading order means simply adding eqs. (3.29) and (3.43). Once this is done, the resulting potential can be adjusted to continue having a local minimum at $\varphi \simeq \varphi_{m}$ for which $V$ vanishes or is positive. The asymptotic region at $|\varphi| \rightarrow \infty$, where $V \rightarrow 0$, is then separated from this minimum by a potential barrier, making the local minimum unstable to tunnelling. However the barrier width can easily be wide enough to make the lifetime of this tunnelling long enough to be stable for all practical purposes.

An alternative tack on uplifting is to try to do so using physics which does not itself badly break supersymmetry (unlike the D3-bar) and so which can be described purely within the framework of $4 \mathrm{D} N=1$ supergravity. One way to do so is to turn on magnetic fluxes on some of the $\mathrm{D} 7 \mathrm{branes}$, since this allows supersymmetry to be broken in a parametrically small way. The resulting energy is positive, and appears within the low-energy supergravity as a contribution to the positive potential, $V_{D}$, of eq. (3.28) [52]. It can be tricky to realize this mechanism explicitly in brane constructions, due to the need to ensure that the low-energy theory does not acquire new light fields, and so modify the KKLT stabilization argument [53]. (See also [54] for a different uplifting proposal.)

\subsection{Some Inflationary Models}

With this lengthy preamble it is now possible to describe briefly some of the inflationary proposals that have been made to date. The examples presented here are not meant to be exhaustive, but instead are chosen to illustrate some of the insights which stand to be gained by making a connection between inflation and string theory.

\section{Racetrack Models}

The simplest approach is to ask if moduli themselves can play the role of the inflaton $[35,55]$. More precisely, do the $4 \mathrm{D}$ effective potentials for those vacua having a small number of moduli have regions for which the slow-roll conditions are satisfied? Although this appears not to be possible for the simplest single-modulus example examined by KKLT, it does seem to be possible for only marginally more complicated cases having two complex moduli, $\varphi^{1}$ and $\varphi^{2}$ [56]. 


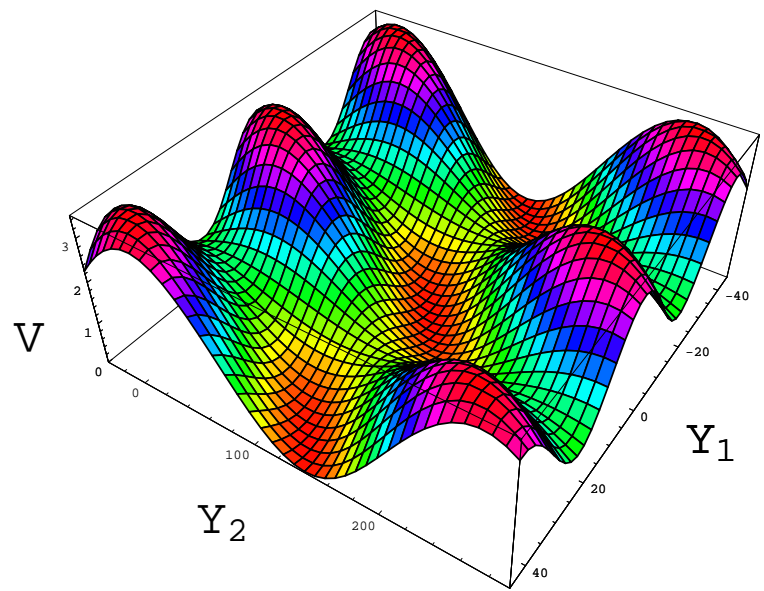

Figure 9: A sketch of the scalar potential as a function of the imaginary parts of the two moduli once the real parts are minimized, for the $\mathbb{P}_{[1,1,1,6,9]}^{4}$ model of ref. [56].

The simplest such an example is based on the Calabi-Yau manifold $\mathbb{P}_{[1,1,1,6,9]}^{4}$, which has a Kähler potential of the form of eq. (3.36) [57], with $k_{2}=1$. The non-perturbative superpotential for this case may also be computed, and is given by

$$
W=W_{0}+A e^{-a \varphi^{1}}+B e^{-b \varphi^{2}},
$$

for calculable constants $A, B, a$ and $b$. Finally, motivated by what would arise in the presence of a D3, the uplifting potential can be taken to be $V_{\overline{D 3}}=\mathscr{E} / \mathscr{V}_{6}^{2}$. As may be seen from Figure 9 , the scalar potential which results has a complicated form as a function of the four real fields, $\operatorname{Re} \varphi^{i}$ and $\operatorname{Im} \varphi^{i}$. Although inflation is not generic for this potential, a numerical search shows that it can occur for specific choices for the various parameters appearing within the superpotential [56]. It is not yet known whether the precise values required can plausibly arise from explicit choices for the underlying Calabi-Yau geometry.

This example - called 'Better' Racetrack Inflation - already teaches us a number of things about string inflation. First, the inflationary trajectories generically involve complicated motions in the 4-dimensional field space, which are not well described by having only the imaginary or real part of one of the moduli $\varphi^{i}$ evolving with all of the others held fixed. However, as Figure 10 shows, because these fields are typically rolling roughly in a fixed direction over the comparatively short interval of horizon exit, its observational predictions (such as a scalar spectral index $n_{s} \simeq 0.95$ ) are nonetheless well captured by a single-field estimate. Because inflation occurs near the top of a saddle point for $V$, the relevant single-field model is in this case of the small-field form. This, together with the generic decoupling of high-energy modes which is a feature of the effective field theories during inflation [20,21], gives confidence that string modifications do not undermine the basic observational evidence that inflation may have taken place.

Another important feature of the Racetrack models is their strong sensitivity to the parameters chosen for the superpotential. The very existence of a slow roll can be destroyed merely by varying 


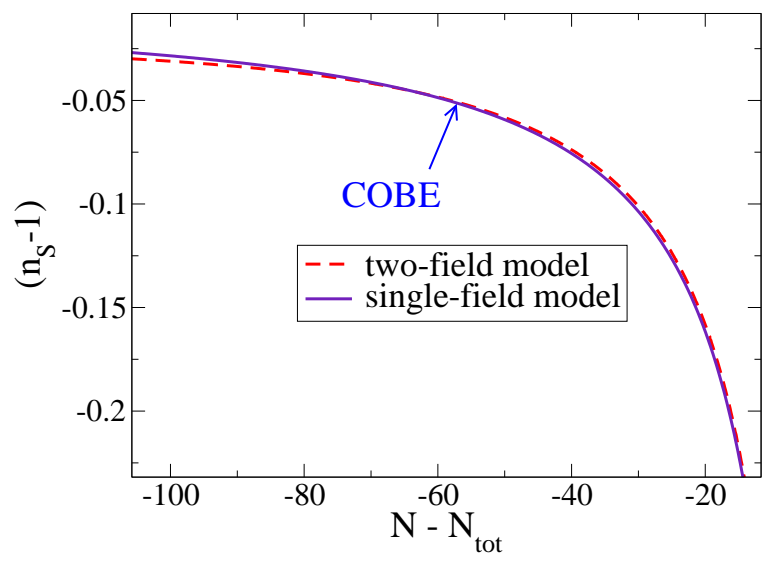

Figure 10: A comparison of a single-field calculation of the scalar spectral index with the full result for the $\mathbb{P}_{[1,1,1,6,9]}^{4}$ model of ref. [56].

these parameters by a percent or less [56]. This is similar to what is encountered in simple singlefield models, where potential parameters must be adjusted with similar accuracy in order to ensure both $\varepsilon$ and $\eta$ are small enough to provide sufficient inflation. The slightly more complicated model described in the next section may be more successful on this particular score.

\section{Kähler Moduli Inflation}

Kähler Moduli Inflation [58,59] works within a class of Type IIB string vacua that are interesting in their own right, which differ from the KKLT minima by not assuming $W_{0}$ to be anomalously small. In this case the perturbative $\alpha^{\prime}$ corrections to $K$ are no longer negligible, and their presence gives rise to new minima for the potential. In order to trust these new minima within the context of the $\alpha^{\prime}$ expansion, it is necessary to work with Calabi-Yau vacua having more than one modulus [60]. Among their attractive phenomenological features are the enormous range of volumes, $\mathscr{V}_{6}$, which are possible for the underlying Calabi-Yau space (due to the exponential dependence of $\mathscr{V}_{6}$ on the parameters of the compactification), as well as the fact that supersymmetry is not preserved at the minimum (even before uplifting by a $\overline{\mathrm{D} 3}$ brane) since $D_{i} W\left(\varphi_{m}\right) \neq 0$.

The simplest models of this class known to have scalar potentials that inflate involve three moduli, $\varphi^{i}$ with $i=1,2,3$. Their Kähler potential is as given in eq. (3.36), supplemented by the perturbative correction of eq. (3.37), and their superpotential is

$$
W=W_{0}+\sum_{i} A_{i} e^{-a_{i} \varphi^{i}}
$$

The full scalar potential is then obtained by combining the resulting supersymmetric expression, $V_{F}$, with an uplifting term of the form $V_{\overline{D 3}} \propto 1 / \mathscr{V}_{6}^{2}$.

Denoting $\tau^{i}=\operatorname{Re} \varphi^{i}$, this potential can lead to inflation in the regime where $\tau^{3}$ is much larger than the others, with $e^{-a_{3} \tau^{3}} \sim O\left(\mathscr{V}_{6}^{-2}\right) \ll 1$. In this case the motion largely involves only $\tau^{3}$, with 
$V$ approximated by the expression

$$
V \simeq V_{0}-C\left(\tau_{c}^{3}\right)^{4 / 3} \exp \left[-c\left(\tau_{c}^{3}\right)^{4 / 3}\right]
$$

where $\tau_{c}^{3}$ denotes the canonically normalized variable along the $\tau^{3}$ direction [58]. Slow roll in this case requires only $\tau_{c}^{3}$ to be sufficiently large, which lies within the domain of the approximations used to compute $V$. Furthermore, since the roll is towards smaller values of $\tau_{c}^{3}$, eventually this condition fails and corrections to eq. (3.46) become important, providing an exit from inflation.

The attractive new feature of this model is the insensitivity of the slow-roll conditions from specific choices for the parameters (like $c$ and $C$ ) that are explicitly given in the potential. Whether it is similarly independent of other implicit choices of parameters, such as those possibly arising once string loop corrections are incorporated into the potential, is not yet known.

\section{Inflation due to Brane Motion}

Another broad class of inflationary constructions within string theory relies on using the positions of various branes as the inflaton [23]. In particular, using the separation between an antibrane and a brane (or configuration of other branes) as the inflaton turns these models into useful tools for exploring inflationary possibilities in string theory, by allowing supersymmetry breaking to be incorporated in a calculable way [24].

Within this framework inflaton dynamics is governed by the potential describing the various forces acting between the various branes. Finding inflation is difficult for these models because although inter-brane forces typically fall off like a power of the inter-brane separation, branes can never get far enough apart from one another within the extra dimensions to allow this falloff to become shallow enough for a slow roll to occur [24]. This observation has led to the proposal of a variety of mechanisms for achieving sufficiently weak inter-brane forces, involving the interactions of branes oriented at angles to one another [37], dual formulations of branes at angles [36], D3 branes falling towards D7 branes [61], and so on [62]. These models usually resemble Hybrid Inflation in their predictions, because of the appearance of an open-string tachyon (expressing their instability towards annihilation) once the branes approach to within the string length of one another.

Since brane positions, $z^{i}$, appear in the low-energy effective theory together with other moduli, real progress has become possible once these ideas were embedded into a framework which stabilizes the various moduli [63]. The simplest proposal starts with the basic one-modulus model defined with extra dimensions having a strongly warped throat à la KKLT. Brane dynamics is then added by including a mobile D3 brane which is free to move, and is drawn down the throat by its attraction towards the anti-D3 which sits at its tip. The trick to make this precise is to cast both the modulus-stabilizing and inter-brane forces in terms of an effective 4D supergravity, since this gives control over the corrections which are possible to the leading semiclassical approximations.

A D3 brane added to a Type IIB vacuum in this way changes both the Kähler potential and superpotential of the low-energy 4D supergravity, and each of these changes describes a different kind of inter-brane force. Modifications making the Kähler function depend on the presence of the 
3-brane position, $z^{i}$, modifies eq. (3.33) to take the form

$$
K(\varphi, z, \bar{\varphi}, \bar{z})=-3 \ln [\varphi+\bar{\varphi}-\kappa k(z, \bar{z})],
$$

where $\kappa$ is a constant and $k(z, \bar{z})$ is the Kähler potential for the Calabi-Yau metric, $g_{m n}(y)$, itself, in the sense that $g_{i \bar{j}}(z, \bar{z})=\partial_{i} \partial_{\bar{j}} k$ for an appropriate choice of coordinates. The correctness of this form for the Kähler potential may be inferred by requiring agreement with the dimensionally-reduced kinetic term, eq. (3.16) for the D3-brane [64], and requiring that the supersymmetric potential for the modulus vanishes identically when $W=W_{0}$ (see Exercise 14).

Exercise 14: Show that the Kähler potential, $K$, of eq. (3.47) satisfies the no-scale identity, eq. (3.35), and so $V_{F}=0$ when the superpotential is constant, $W=W_{0}$.

The potential, eq. (3.47), describes a force on the D3 brane once the moduli get stabilized because once $W$ depends on $\varphi, V_{F}$ acquires nontrivial dependence on $z^{i}$. Physically, the absence of such a potential when $W=W_{0}$ expresses the absence of a net static force between the D3 and the other branes present in the extra dimensions. However this absence of a net force happens due to the cancelling (due to the supersymmetry of the background geometry) of a variety of interbrane forces having their origin in the exchange of massless bulk states (gravitons, dilatons, and so on). However, if the D3 is moved within the extra dimensions the distribution of forces acting on the branes adjusts, as they try to maintain their cancellation at the new position of the D3. This adjustment in turn causes the volume modulus, $\varphi$, to change, as the internal geometry responds to new distribution of forces. The change of the extra-dimensional volume costs no energy so long as the breathing mode is a modulus. But once this modulus has been stabilized (by having $W$ depend on $\varphi$ ) the energy cost associated with this adjustment induces a force (expressed by the interactions between $\varphi$ and $z^{i}$ in $K$ ) which tends to localize the D3 at a specific position within the extra dimensions.

Modifications that introduce a $z^{i}$ dependence directly into $W$ describe a second kind of force experienced by the D3. This force arises due to the back-reaction of the D3 onto the background extra-dimensional geometry, since this changes the volume of the cycle wrapped by any D7 branes, and thereby changes the gauge couplings of the interactions on these branes (such as those which generate $W_{n p}$ ). In the low-energy supergravity this effect appears as a calculable $z$-dependence to the constant $A=A(z)$ appearing in eq. (3.38) [65].

\section{KKLMMT-type Models}

Ref. [63] performed the first search for inflation, using eq. (3.47) with the non-perturbative superpotential, eq. (3.38), together with the uplifting term, eq. (3.43). They found that although the strong warping in the throat tends to favor a slow D3 roll, the coupling between $z^{i}$ and $\varphi$ embodied by eq. (3.47) generically steepens this potential sufficiently to prevent inflation's occurrence.

Inflation within this context requires a more detailed balancing of the forces acting on the D3 brane. One way this might occur would arise if the above-mentioned volume-stabilization force were to localize the brane at a position removed from the tip of the throat, because in this case the 


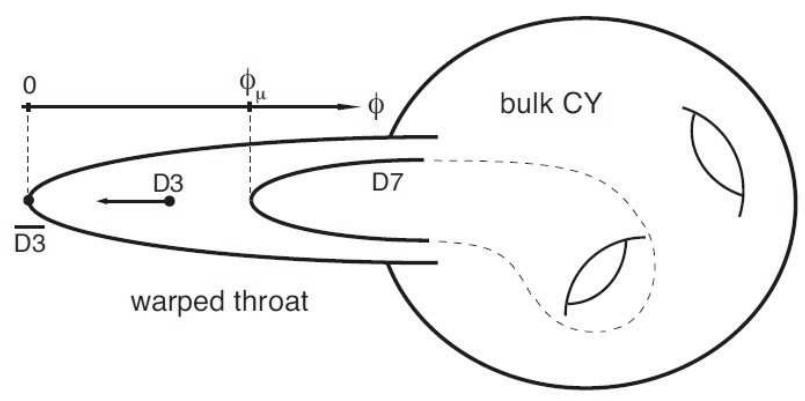

Figure 11: A sketch of a D7 descending partially into a warped throat, as assumed in the inflationary scenario of ref. [68].

pull of the mobile D3 towards this point can be balanced against its Coulomb attraction towards the anti-D3 which is situated at the throat's tip. In this case a slow roll can occur when the D3 is close to where these forces balance, and ends if the D3 slowly rolls off as it succumbs to its attraction to the anti-D3 brane [66]. As mentioned earlier, the observational predictions for this inflation fall into the category of Hybrid Inflation, with the two fields physically corresponding to the interplay between the inter-brane separation and an open-string tachyon which describes the instability towards mutual annihilation. As a result models of this form exist for which both $n_{s}>1$ [66] and $n_{s}<1$ [67].

However, from the point of view of providing a string embedding of inflation, this kind of picture suffers from two drawbacks. First, it assumes the forces on the D3 brane stabilize it away from the throat's tip, without providing an explicit extra-dimensional construction which does so. Secondly, by relying on the brane-antibrane Coulomb force, it steps outside of the low-energy 4D supergravity approximation, and so makes difficult the quantification of the possible corrections to the semiclassical approximation which might arise. ${ }^{6}$

A more convincing stringy grounding of this type of inflation in string theory instead requires a description of all forces in terms of the low-energy supergravity. This has recently become possible using the $z$-dependence of the superpotential [65] which arises when a D7 extends partially down into a warped throat along particular kinds of cycles (see Figure 11). In this case, the resulting $z$-dependence of $W$ shows that D3 branes in the same throat can experience a balance of forces towards the tip and towards the D7 brane, allowing slow-roll inflation to occur for some choices of the various parameters describing the underlying vacuum [68].

\section{Brane Annihilation and Reheating}

Once the D3 brane and the anti D3-brane come to within the string length of one another, stringy physics intervenes and the two branes annihilate one another. This annihilation process has two potentially important observational implications. First, annihilation takes place through having

\footnotetext{
${ }^{6}$ Of course, this objection also applies to most of the other proposed brane-based inflationary mechanisms.
} 
their world sheets fragment into pairs a D1 and $\overline{\mathrm{D} 1}$ branes (or, D-strings), which then find one another and continue to annihilate in a cascade towards the vacuum state [24]. The competition of this annihilation rate with the expansion of the universe can be described in a manner very similar to the Kibble process describing phase transitions, familiar to cosmologists. This allows a quantitative estimate of the number of D1 and $\overline{\mathrm{D} 1}$ that fail to find their anti-branes to annihilate, with the result that they can be abundant enough to be detectable as cosmic strings in the present universe [69]. Furthermore (although this depends more on the details of the underlying Calabi-Yau geometry) these strings can be stable enough to avoid having decayed during the intervening epochs [70]. The observation of such cosmic strings together with inflation would provide compelling circumstantial evidence for brane-based inflation.

The second implication of annihilation is the mechanism it provides for reheating the later universe [24], by liberating the brane tensions which provide the underlying inflationary energy density. Once liberated, one must ask whether this energy can get funnelled efficiently enough into observable low-energy degrees of freedom to provide sufficient reheating. Since the observable degrees of freedom in these models tend to reside on other, spectator, branes, a potential danger here is that the released energy is dumped too efficiently into invisible, bulk degrees of freedom rather than into observable modes. However, an important observation [71] is that strong warping can help with the efficiency of energy transfer into the observed sector, provided that this observed sector resides at the tip of a strongly warped region (as tends to be required in any case by particle physics issues, like the Hierarchy Problem). This low-energy mechanism is supported, with some caveats, by the subsequent more detailed string calculations [72].

\section{DBI Inflation: Beyond Slow Rolls}

A related string-based inflationary proposal, again based on brane motion, differs from all of the others by not relying on the usual slow-roll approximation, and so also has a somewhat different observational signature. In this model — known as DBI Inflation - a D3 brane is again envisioned to roll down a strongly warped throat, attracted to an anti-D3 at the tip, but the motion is taken to be relativistically rapid rather than slow. Paradoxically, the energy of such a system can produce accelerated inflationary expansion, despite the motion being the opposite of a slow roll [73].

The starting point for this proposal is the action for a relativistically moving D3 brane moving through a throat, and with a cosmological 4D metric,

$$
\mathrm{d} s^{2}=h^{-1 / 2}(y)\left[-\mathrm{d} t^{2}+a^{2}(t) \mathrm{d} \vec{x}^{2}\right]+h^{1 / 2}(y) g_{m n}(y) \mathrm{d} y^{m} \mathrm{~d} y^{n} .
$$

Denoting the distance to the brane from the throat's tip by $q(t)$, the brane action takes the form

$$
S=-\int \mathrm{d}^{4} x a^{3}\left[\frac{T_{3}}{h(q)}\left(\sqrt{1-h(q) \dot{q}^{2} / T_{3}}-1\right)+V(q)\right],
$$

where $h(q) \simeq b^{4} / q^{4}$ in the throat. The square-root term in the square brackets represents the contribution of the first (Dirac-Born-Infeld, or DBI) term of eq. (3.16), while the second (-1) term is due to the Chern-Simons coupling (i.e. to $C$ of eq. (3.16)). Notice that these cancel when $\dot{q}^{2}=0$, showing the above-mentioned absence of a static force on the D3. In the potential, 
$V(q)=V_{0}+\frac{1}{2} m^{2} q^{2}-k / q^{4}, V_{0}$ describes the tension of other branes, $\frac{1}{2} m^{2} q^{2}$ phenomenologically describes the forces, discussed above, which act to localize the brane at the throat's tip, and $k / q^{4}$ describes the Coulomb attraction towards the antibrane, also located at the tip.

Notice that in the limit of a slow roll, when $\dot{q}^{2}$ is small, the lagrangian density of eq. (3.49) reduces to a standard non-relativistic point-particle action, $\mathscr{L} \simeq a^{3}\left[\frac{1}{2} \dot{q}^{2}-V\right]$. The full action provides the relativistic generalization, and takes the form of the action for a relativistic point particle, but with a speed, $v^{2} / c^{2}=h(q) \dot{q}^{2} / T_{3}$. Some comment is required about the validity of using the full form of eq. (3.49), including the full structure of the square root, given that this cannot be regarded as a standard expansion in derivatives as typically arises at low energies. Is it consistent to drop all higher derivatives (like $\ddot{q}$ ) in $S$ while keeping all powers of $\dot{q}^{2}$ all higher derivatives?

The relativistic particle action is one of the few cases where it can be a consistent approximation to trust the entire square-root action while neglecting higher time derivatives. It is selfconsistent to do so because as the motion becomes more and more relativistic, $v^{2} / c^{2}$ asymptotes to 1 and the equations of motion imply the higher derivatives go to zero. When $h$ is a constant the same is true for the DBI action, eq. (3.49), since its equations of motion imply that $\ddot{q}$ and higher derivatives become suppressed in the ultra-relativistic limit. The same should also hold if the spatial variation of $h(q)$ is sufficiently slow.

How can this kind of relativistic motion be consistent with a lengthy period of inflation and the equation of state, $p<-\frac{1}{3} \rho$ (and so potential-energy domination) which inflation requires? The answer is in the warping: $(i)$ when passing through a strongly warped region $h \gg 1$, and so $\dot{q}^{2}$ can be small (so inflation last a long time) even if $h \dot{q}^{2} / T_{3}$ is $O(1)$; and (ii) because the kinetic energy's pre-factor of $1 / h$ suppresses it relative to $V$ in strongly-warped regions, even if the motion is relativistic.

Because the motion is not slow, the predictions of DBI inflation cannot be inferred using the slow-roll expressions of the previous sections, which are entirely expressed in terms of the derivatives of the scalar potential. Instead we must generalize to define slow-roll parameters that rely only on what is important: the approximate constancy of $H$ during inflation. To this end define the generalized slow-roll parameters $\tilde{\varepsilon}$ and $\tilde{\eta}$ by [13]

$$
\tilde{\varepsilon} \equiv-\frac{\dot{H}}{H^{2}} \quad \text { and } \quad \tilde{\eta} \equiv \frac{\dot{\tilde{\varepsilon}}}{\tilde{\varepsilon} H}
$$

and so on, for successively higher derivatives.

To make contact between these definitions and the action, consider the general situation where

$$
S=\int \mathrm{d}^{4} x a^{3} p(q, \mathscr{X}),
$$

where $\mathscr{X}=\frac{1}{2} \dot{q}^{2} / T_{3}$. The action of interest, eq. (3.49), corresponds to the special case where

$$
p(q, \mathscr{X})=-\frac{T_{3}}{h(q)}[1-2 h(q) \mathscr{X}]^{1 / 2}+\frac{T_{3}}{h(q)}-V(q) .
$$

The energy density computed from this action is then

$$
\rho(q, \mathscr{X})=2 \mathscr{X} p, \mathscr{X}-p
$$


and it is useful to define the 'speed of sound',

$$
c_{s}^{2}=\frac{p, \mathscr{X}}{\rho_{, \mathscr{X}}}=\frac{p_{, \mathscr{X}}}{p_{, \mathscr{X}}+2 \mathscr{X} p, \mathscr{X} \mathscr{X}},
$$

which when specialized to the action, eq. (3.49), becomes

$$
c_{s}^{2}=1-2 h \mathscr{X}=\frac{1}{\gamma^{2}},
$$

where the relativistic $\gamma$ factor is defined, as usual, by $\gamma \equiv[1-2 h \mathscr{X}]^{-1 / 2} \geq 1$, with relativistic motion characterized by $\gamma \gg 1$. Using these expressions in the Friedmann and Raychaudhuri equations, eqs. (1.5) and (1.6), to evaluate $H$ and its derivatives, then gives, for instance

$$
\tilde{\varepsilon}=\frac{\mathscr{X} p_{, \mathscr{X}}}{M_{p}^{2} H^{2}}=\frac{3 \mathscr{X} p_{, \mathscr{X}}}{2 \mathscr{X} p_{, \mathscr{X}}-p},
$$

which reduces in the non-relativistic case, $p \simeq T_{3} \mathscr{X}-V$, to the usual slow-roll result $\tilde{\varepsilon} \simeq \frac{3}{2} \dot{q}^{2} / V \simeq$ $\varepsilon$.

The expressions for the amplitude of primordial fluctuations then generalize from the usual slow-roll results, eqs. (2.44) and (2.50), to

$$
\Delta_{\Phi}^{2}=\frac{H^{2}}{8 \pi^{2} M_{p}^{2} \tilde{\varepsilon} c_{s}} \quad \text { and } \quad \Delta_{T}^{2}=\frac{2 H^{2}}{\pi^{2} M_{p}^{2}} .
$$

From these the following formula for the spectral index are obtained

$$
n_{s}-1=-2 \tilde{\varepsilon}-\tilde{\eta}-s, \quad n_{T}=-2 \tilde{\varepsilon} \quad \text { and } \quad r=-16 \tilde{\varepsilon} c_{s},
$$

where the new contributions come from the appearance of $c_{s}$, and the parameter $s$ is defined by

$$
s \equiv \frac{\dot{c}_{s}}{c_{s} H} .
$$

The previous slow-roll formulae are obtained in the limit $c_{s}=1$, and so $s=0$.

There is an important observational way to distinguish between inflation of this type and that arising from an honest-to-God slow roll [74]. This is because the fluctuations predicted by DBI inflation are not Gaussian when the brane motion is in the ultra-relativistic limit, $\gamma \gg 1$. Although it goes beyond the scope of these lectures, the deviation from Gaussianity can be quantified by a dimensionless parameter $f_{N L}$, which vanishes for purely Gaussian fluctuations. Observations of the microwave background are consistent with Gaussian fluctuations, and currently constrain $-256<f_{N L}<332$. For comparison, the prediction of DBI inflation is $f_{N L} \simeq 0.32 \gamma^{2}$, implying the observational bound $\gamma \lesssim 32$.

\section{What We've Learned}

Recent years have seen some progress in trying to embed inflation into a string theoretic framework, recently stimulated by strides taken in understanding how moduli are fixed for Type IIB string vacua, and rapid progress continues to be made. Although it is still early days, string theory has already offered some insights into how inflation might work within a fundamental context. Some of these are, in a nutshell: 
- Single-Field Slow Roll Models: Single-field slow-roll models (and simple multi-field models, like Hybrid Inflation) capture most of the predictions of the known string-inflationary scenarios. Partly this is because the tools available only allow the exploration of string dynamics when it is described by an effective 4D theory. But it is also true that these low-energy field theories typically involve many light scalars during the inflationary epoch, and although it is necessary to properly follow the dynamics of these extra scalars when finding inflation, their presence often does not crucially alter the observational predictions for the spectrum of primordial fluctuations. This gives some assurance that we are not being led far astray when analyzing cosmological data using simple single-field models.

- Decoupling and Robustness: Even though there are many heavy fields in addition to the inflationary sector, all the evidence is that in string theory those with masses much greater than $H_{I}$ decouple and so have a negligible effect during horizon exit [21]. As a result it suffices to describe inflation purely in terms of the relevant inflaton physics at the inflationary scale. It can be possible to have decoupling break down, such as by having nominally heavy particles become light; by having some fields evolve non-adiabatically; or by having inflation start just before horizon exit. But the current evidence is that when it does so, it does so in the usual way that time-dependent effective field theories do [20].

- New Signatures: Although inflation, where found so far in string theory, is well-described by a $4 \mathrm{D}$ effective field theory, several inflationary scenarios do differ in their implications from simple slow-roll models. Brane-antbrane inflationary mechanisms can also give rise to relic cosmic strings $[24,69,70]$, and the detection of these would provide considerable circumstantial evidence for this kind of mechanism. DBI inflationary models can predict non-Gaussian primordial fluctuations, and their detection would definitively rule out inflation due to a single-field slow-roll mechanism [73].

- Naturalness: For most stringy scenarios parameters in the potentials must be adjusted in order to ensure a slow roll, at a level which is consistent with the adjustments that are required in simple single-field models. But two approaches may prove to be more promising in this regard: Kähler Moduli Inflation [58], and DBI inflation [73], since these may produce inflation more robustly than other models. Whether these models definitively emerge as more natural than others remains the subject of active current study.

- Reheating: It is a bit premature to fully address reheating issues, since no string model has yet been constructed which provides both a convincing inflationary picture as well as a properly formulated Standard Model sector to describe particle physics, including a proper understanding of the Hierarchy Problem $[66,75]$. Both are required to address reheating after inflation, but the first indications are that stringy inflationary scenarios provide a number of novel challenges and opportunities for reheating [71, 72].

Further insights are certain to emerge as the inflationary options become better investigated. 


\section{Acknowledgements}

I would like to thank the organizers of these schools for their kind invitation to present these lectures, and to thank my collaborators for their help in understanding the many thorny inflationary issues. My research has been supported by the Natural Sciences and Engineering Research Council of Canada, by McMaster University and by the Killam Foundation. Research at Perimeter Institute is supported in part by the Government of Canada through NSERC and by the Province of Ontario through MEDT.

\section{References}

[1] A. H. Guth, Phys. Rev. D23 (1981) 347; A. D. Linde, Phys. Rev. B108 (1982) 389; A. Albrecht and P. J. Steinhardt, Phys. Rev. Lett. 48 (1982) 1220.

[2] S. Weinberg, Gravitation and Cosmology: Principles and Applications of the General Theory of Relativity, Wiley 1972; E. W. Kolb and M. S. Turner, The Early Universe, Addison-Wesley (1990); P.J.E. Peebles, Principles of Physical Cosmology, Princeton University Press (1993); B. Ryden, Introduction to Cosmology, Pearson Education 2003; S. Dodelson, Modern Cosmology, Academic Press 2003.

[3] V. Mukhanov, Physical Foundations of Cosmology, Cambridge University Press (2005).

[4] D. N. Spergel et al. [WMAP Collaboration], "First Year Wilkinson Microwave Anisotropy Probe (WMAP) Observations: Determination of Cosmological Parameters,” Astrophys. J. Suppl. 148 (2003) 175 [astro-ph/0302209];

[5] D. N. Spergel et al., "Wilkinson Microwave Anisotropy Probe (WMAP) three year results: Implications for cosmology," [astro-ph/0603449].

[6] A. Linde, Particle Physics and Inflationary Cosmology, Harwood Academic Publishers (1990).

[7] A. R. Liddle and D. H. Lyth, Cosmological Inflation and Large-Scale Structure, Cambridge University Press (2000).

[8] A. Linde, arXiv:0705.0164 [hep-th].

[9] A. H. Guth and S. Y. Pi, Phys. Rev. D 32, 1899 (1985); M. A. Sakagami, Prog. Theor. Phys. 79, 442 (1988);

[10] L. P. Grishchuk and Y. V. Sidorov, Class. Quant. Grav. 6 (1989) L161; R. H. Brandenberger, R. Laflamme and M. Mijic, Mod. Phys. Lett. A 5, 2311 (1990); E. Calzetta and B. L. Hu, Phys. Rev. D 52, 6770 (1995) [gr-qc/9505046]; D. Polarski and A. A. Starobinsky, Class. Quant. Grav. 13, 377 (1996) [gr-qc/9504030]; F. C. Lombardo and D. Lopez Nacir, Phys. Rev. D 72, 063506 (2005) [gr-qc/0506051]; C.P. Burgess, R. Holman and D. Hoover, [astro-ph/0601646].

[11] L. P. Grishchuk and Y. V. Sidorov, Phys. Rev. D 42, 3413 (1990); A. Albrecht, P. Ferreira, M. Joyce and T. Prokopec, Phys. Rev. D 50, 4807 (1994) [astro-ph/9303001]; C. Kiefer, D. Polarski and A. A. Starobinsky, Int. J. Mod. Phys. D 7, 455 (1998) [gr-qc/9802003]; J. Lesgourgues, D. Polarski and A. A. Starobinsky, Nucl. Phys. B 497, 479 (1997) [gr-qc/9611019]. 
[12] L. Randall and S. D. Thomas, Nucl. Phys. B 449 (1995) 229 [hep-ph/9407248]; D. H. Lyth and E. D. Stewart, Phys. Rev. D 53 (1996) 1784 [hep-ph/9510204]; T. Barreiro, E. J. Copeland, D. H. Lyth and T. Prokopec, Phys. Rev. D 54 (1996) 1379 [hep-ph/9602263]; T. Matsuda, Phys. Lett. B 486 (2000) 300 [hep-ph/0002194]; M. Postma, JCAP 0403 (2004) 006 [astro-ph/0311563]; R. Allahverdi, K. Enqvist, J. Garcia-Bellido and A. Mazumdar, Phys. Rev. Lett. 97 (2006) 191304 [hep-ph/0605035]; D. H. Lyth, JCAP 0704 (2007) 006 [hep-ph/0605283]; R. Allahverdi, K. Enqvist, J. Garcia-Bellido, A. Jokinen and A. Mazumdar, JCAP 0706 (2007) 019 [hep-ph/0610134]; R. Allahverdi, A. R. Frey and A. Mazumdar, Phys. Rev. D 76 (2007) 026001 [hep-th/0701233]; R. Allahverdi, B. Dutta and A. Mazumdar, Phys. Rev. D 75 (2007) 075018 [hep-ph/0702112]; K. Enqvist, L. Mether and S. Nurmi, arXiv:0706.2355 [hep-th].

[13] H. V. Peiris et al., Astrophys. J. Suppl. 148 (2003) 213 [astro-ph/0302225].

[14] V. Barger, H. S. Lee and D. Marfatia, Phys. Lett. B 565 (2003) 33 [hep-ph/0302150]; S. M. Leach and A. R. Liddle, Phys. Rev. D 68 (2003) 123508 [astro-ph/0306305]; L. Alabidi and D. H. Lyth, [astro-ph/0603539]; W. H. Kinney, arXiv:0706.3699 [astro-ph].

[15] A. Albrecht, P. J. Steinhardt, M. S. Turner and F. Wilczek, Phys. Rev. Lett. 48 (1982) 1437; A. Ringwald, Z. Phys. C 34 (1987) 481; S. M. Barr and G. Segre, Phys. Rev. Lett. 62 (1989) 2781; R. Watkins and L. M. Widrow, Nucl. Phys. B 374 (1992) 446; N. Deruelle, J. Garriga and E. Verdaguer, Phys. Rev. D 43 (1991) 1032; L. Kofman, A. D. Linde and A. A. Starobinsky, Phys. Rev. Lett. 73 (1994) 3195 [hep-th/9405187]; Phys. Rev. D 56 (1997) 3258 [hep-ph/9704452]; Y. Shtanov, J. H. Traschen and R. H. Brandenberger, Phys. Rev. D 51 (1995) 5438 [hep-ph/9407247]; D. Boyanovsky, M. D'Attanasio, H. J. de Vega, R. Holman, D. S. Lee and A. Singh, Phys. Rev. D 52 (1995) 6805 [hep-ph/9507414]; R. Allahverdi and B. A. Campbell, Phys. Lett. B 395 (1997) 169 [hep-ph/9606463]; A. Dolgov, K. Freese, R. Rangarajan and M. Srednicki, Phys. Rev. D 56 (1997) 6155 [hep-ph/9610405]; T. Falk, K. A. Olive, L. Roszkowski, A. Singh and M. Srednicki, Phys. Lett. B 396 (1997) 50 [hep-ph/9611325]; S. A. Ramsey and B. L. Hu, Phys. Rev. D 56 (1997) 678 [Erratum-ibid. D 57 (1998) 3798] [hep-ph/9706207]; V. Zanchin, A. . J. Maia, W. Craig and R. H. Brandenberger, Phys. Rev. D 57 (1998) 4651 [hep-ph/9709273]; D. J. H. Chung, E. W. Kolb and A. Riotto, Phys. Rev. D 60 (1999) 063504 [hep-ph/9809453]; F. Finelli and R. H. Brandenberger, Phys. Rev. Lett. 82 (1999) 1362 [hep-ph/9809490]; R. Allahverdi, B. A. Campbell and J. R. Ellis, Nucl. Phys. B 579 (2000) 355 [hep-ph/0001122]; and so on.

[16] A. D. Linde, Phys. Lett. B 162 (1985) 281; A. D. Linde, D. A. Linde and A. Mezhlumian, Phys. Rev. D 49, 1783 (1994) [gr-qc/9306035]; A. D. Linde, Phys. Lett. B 327, 208 (1994) [astro-ph/9402031]; A. Vilenkin, Phys. Rev. Lett. 72, 3137 (1994) [hep-th/9402085]; N. Kaloper, M. Kleban, A. Lawrence, S. Shenker and L. Susskind, JHEP 0211 (2002) 037 [hep-th/0209231].

[17] P. J. Steinhardt, In: The Very Early Universe, ed. G.W. Gibbons, S.W. Hawking and S.Siklos, Cambridge University Press, (1983); A. D. Linde, Cambridge University preprint Print-82-0554 (1982); A. Vilenkin, Phys. Rev. D 27, 2848 (1983).

[18] A. Linde, "Inflation and string cosmology," eConf C040802 (2004) L024 [J. Phys. Conf. Ser. 24 (2005) 151] [hep-th/0503195]; C. P. Burgess, "Inflatable string theory?," Pramana 63 (2004) 1269 [hep-th/0408037]; "Strings, Branes and Cosmology: What Can We Hope to Learn?" [hep-th/0606020]; F. Quevedo, Class. Quant. Grav. 19 (2002) 5721, [hep-th/0210292]; AIP Conf. Proc. 743 (2005) 341. S. H. Henry Tye, [hep-th/0610221]; J. M. Cline, "String cosmology," [hep-th/0612129]; R. Kallosh, [hep-th/0702059]. 
[19] J. Martin and R. H. Brandenberger, Phys. Rev. D 63 (2001) 123501 [hep-th/0005209];

R. H. Brandenberger and J. Martin, Mod. Phys. Lett. A 16 (2001) 999 [astro-ph/0005432]; R. Easther,

B. R. Greene, W. H. Kinney and G. Shiu, Phys. Rev. D 64, 103502 (2001) [hep-th/0104102].

[20] C. P. Burgess, J. M. Cline, F. Lemieux and R. Holman, JHEP 0302 (2003) 048 [hep-th/0210233];

C. P. Burgess, J. M. Cline and R. Holman, JCAP 0310 (2003) 004 [hep-th/0306079].

[21] N. Kaloper, M. Kleban, A. E. Lawrence and S. Shenker, Phys. Rev. D 66 (2002) 123510 [hep-th/0201158].

[22] D. H. Lyth, Phys. Rev. Lett. 78 (1997) 1861 [hep-ph/9606387].

[23] G. R. Dvali and S. H. H. Tye, Phys. Lett. B 450 (1999) 72 [hep-ph/9812483].

[24] C. P. Burgess, M. Majumdar, D. Nolte, F. Quevedo, G. Rajesh and R. J. Zhang, JHEP 0107 (2001) 047 [hep-th/0105204].

[25] G. R. Dvali, Q. Shafi and S. Solganik, hep-th/0105203.

[26] D. Baumann and L. McAllister, Phys. Rev. D 75 (2007) 123508 [hep-th/0610285].

[27] M. Gasperini and G. Veneziano, Astropart. Phys. 1 (1993) 317 [hep-th/9211021]; C. Angelantonj, L. Amendola, M. Litterio and F. Occhionero, Phys. Rev. D 51 (1995) 1607 [astro-ph/9501008];

J. Khoury, B. A. Ovrut, P. J. Steinhardt and N. Turok, Phys. Rev. D 64 (2001) 123522

[hep-th/0103239]; P. J. Steinhardt and N. Turok, Phys. Rev. D 65 (2002) 126003

[arXiv:hep-th/0111098]; R. Kallosh, L. Kofman and A. D. Linde, Phys. Rev. D 64 (2001) 123523

[arXiv:hep-th/0104073]; L. Kofman, A. Linde and V. F. Mukhanov, JHEP 0210 (2002) 057

[hep-th/0206088]; R. H. Brandenberger, A. Nayeri, S. P. Patil and C. Vafa, [hep-th/0608121];

N. Kaloper, L. Kofman, A. Linde and V. Mukhanov, JCAP 0610 (2006) 006 [hep-th/0608200].

[28] M. B. Green, J. H. Schwarz and E. Witten, Superstring Theory, Vols. 1 \& 2, Cambridge University Press (1987); J. Polchinski, String Theory, Vols. 1 \& 2 Cambridge University Press (1998); B. Zweibach, A First Course in String Theory, Cambridge University Press (2004); K. Becker, M. Becker and J. H. Schwarz, String theory and M-theory: A modern introduction, Cambridge University Press (2007).

[29] J. Polchinski, "Lectures on D-branes," [hep-th/9611050]; S. Kachru, "Lectures on warped compactifications and stringy brane constructions," [hep-th/0009247].

[30] C. P. Burgess, F. Quevedo, S. J. Rey, G. Tasinato and I. Zavala, JHEP 0210 (2002) 028 [arXiv:hep-th/0207104]; N. Ohta, Phys. Rev. Lett. 91 (2003) 061303 [hep-th/0303238]; Prog. Theor. Phys. 110 (2003) 269 [hep-th/0304172]; C. M. Chen, P. M. Ho, I. P. Neupane, N. Ohta and J. E. Wang, JHEP 0310 (2003) 058 [hep-th/0306291]; C. P. Burgess, C. Nunez, F. Quevedo, G. Tasinato and I. Zavala, JHEP 0308 (2003) 056 [hep-th/0305211]; G. Tasinato, I. Zavala, C. P. Burgess and F. Quevedo, JHEP 0404 (2004) 038 [hep-th/0403156]. N. Ohta, Int. J. Mod. Phys. A 20 (2005) 1 [hep-th/0411230].

[31] A. D. Linde, Phys. Rev. D 49 (1994) 748 [astro-ph/9307002].

[32] Z. Lalak, D. Langlois, S. Pokorski and K. Turzynski, arXiv:0704.0212 [hep-th]; N. Barnaby and J. M. Cline, arXiv:0704.3426 [hep-th].

[33] P. Candelas, G. T. Horowitz, A. Strominger and E. Witten, Nucl. Phys. B 258 (1985) 46. 
[34] I. Antoniadis, C. Bachas, J. R. Ellis and D. V. Nanopoulos, Phys. Lett. B 211 (1988) 393; S. Kalara and K. A. Olive, Phys. Lett. B 218 (1989) 148; A. A. Tseytlin and C. Vafa, Nucl. Phys. B 372 (1992) 443 [hep-th/9109048]; B. A. Campbell, A. D. Linde and K. A. Olive, Nucl. Phys. B 355 (1991) 146; R. Brustein and P. J. Steinhardt, Phys. Lett. B 302 (1993) 196 [hep-th/9212049]; T. Damour and A. Vilenkin, Phys. Rev. D 53 (1996) 2981 [hep-th/9503149].

[35] C. P. Burgess, P. Martineau, F. Quevedo, G. Rajesh and R. J. Zhang, JHEP 0203 (2002) 052 [hep-th/0111025].

[36] A. Avgoustidis, D. Cremades and F. Quevedo, [hep-th/0606031].

[37] J. Garcia-Bellido, R. Rabadan and F. Zamora, JHEP 0201, 036 (2002); N. Jones, H. Stoica and S. H. H. Tye, JHEP 0207, 051 (2002); M. Gomez-Reino and I. Zavala, JHEP 0209, 020 (2002).

[38] L. Pogosian, S. H. H. Tye, I. Wasserman and M. Wyman, Phys. Rev. D 68 (2003) 023506 [Erratum-ibid. D 73 (2006) 089904] [hep-th/0304188]; L. Pilo, A. Riotto and A. Zaffaroni, JHEP 0407 (2004) 052 [hep-th/0401004]; D. H. Lyth and A. Riotto, Phys. Rev. Lett. 97 (2006) 121301 [astro-ph/0607326]; J. E. Lidsey and D. Seery, Phys. Rev. D 75 (2007) 043505 [astro-ph/0610398].

[39] B. C. Da Cunha and E. J. Martinec, Phys. Rev. D 68 (2003) 063502 [hep-th/0303087]; O. DeWolfe, S. Kachru and H. Verlinde, JHEP 0405 (2004) 017 [hep-th/0403123]; N. Iizuka and S. P. Trivedi, theory," [hep-th/0403203]; D. Cremades, F. Quevedo and A. Sinha, JHEP 0510 (2005) 106 [hep-th/0505252]; S. Dimopoulos, S. Kachru, J. McGreevy and J. G. Wacker, [hep-th/0507205]; B. Freivogel, V. E. Hubeny, A. Maloney, R. Myers, M. Rangamani and S. Shenker, JHEP 0603 (2006) 007 [hep-th/0510046]; K. Becker, M. Becker and A. Krause, Nucl. Phys. B715 (2005) 349-371 [hep-th/0501130]; [hep-th/0510066].

[40] S. B. Giddings, S. Kachru and J. Polchinski, Phys. Rev. D66, 106006 (2002); S. Sethi, C. Vafa and E. Witten, Nucl. Phys. B 480 (1996) 213 [hep-th/9606122]; K. Dasgupta, G. Rajesh and S. Sethi, JHEP 9908 (1999) 023 [hep-th/9908088].

[41] L. Randall and R. Sundrum, Phys. Rev. Lett. 83 (1999) 3370-3373, [hep-ph/9905221]; Phys. Rev. Lett. 83 (1999) 4690-4693, [hep-th/9906064].

[42] E. Cremmer, S. Ferrara, C. Kounnas and D.V. Nanonpoulos, Phys. Lett. B133, 61 (1983); E. Witten and J. Bagger, Phys. Lett. B 115 (1982) 202; Phys. Lett. B 118 (1982) 103.

[43] S. Gukov, C. Vafa and E. Witten, Nucl. Phys. B584, 69 (2000).

[44] E. Witten, Phys. Lett. B 155 (1985) 151; C. P. Burgess, A. Font and F. Quevedo, Action For The Superstring," Nucl. Phys. B 272 (1986) 661.

[45] J. Ellis, A.B. Lahanas, D.V. Nanopoulos and K. Tamvakis, Phys. Lett. B134, 429 (1984).

[46] M. T. Grisaru, W. Siegel and M. Rocek, Nucl. Phys. B 159 (1979) 429; E. Witten, Nucl. Phys. B 268 (1986) 79; M. Dine, N. Seiberg, Phys. Rev. Lett. 57 (1986) 21; N. Seiberg, Phys. Lett. B 318 (1993) 469 [hep-ph/9309335]; K. A. Intriligator and N. Seiberg, Nucl. Phys. Proc. Suppl. 45BC (1996) 1 [hep-th/9509066]; C. P. Burgess, C. Escoda and F. Quevedo, JHEP 0606 (2006) 044 [hep-th/0510213].

[47] M. Berg, M. Haack and B. Kors, Phys. Rev. D 71 (2005) 026005 [hep-th/0404087]; M. Berg, M. Haack and B. Kors, JHEP 0511 (2005) 030 [hep-th/0508043]; M. Berg, M. Haack and B. Kors, Phys. Rev. Lett. 96 (2006) 021601 [hep-th/0508171]; A. Westphal, JCAP 0511 (2005) 003 [hep-th/0507079]; 
[48] S. Kachru, R. Kallosh, A. Linde and S. P. Trivedi, Phys. Rev. D 68 (2003) 046005 [ hep-th/0301240]; B. S. Acharya, [hep-th/0212294]; R. Brustein and S. P. de Alwis, Phys. Rev. D 69 (2004) 126006 [hep-th/0402088]; F. Denef, M. R. Douglas, B. Florea, A. Grassi and S. Kachru, [hep-th/0503124].

[49] E. Witten, “Non-Perturbative Superpotentials In String Theory,” Nucl. Phys. B 474, 343 (1996) [hep-th/9604030].

[50] J. P. Derendinger, L. E. Ibáñez and H. P. Nilles, "On The Low-Energy D = 4, N=1 Supergravity Theory Extracted From The D = 10, N=1 Superstring," Phys. Lett. B 155 (1985) 65; M. Dine, R. Rohm, N. Seiberg and E. Witten, "Gluino Condensation In Superstring Models," Phys. Lett. B 156 (1985) 55; C.P. Burgess, J.P. Derendinger and F. Quevedo and M. Quiros,, Ann. Phys. 250 (1996) 193 [hep-th/9505171]; Phys. Lett. B348 (1995) 428, [hep-th/9501065].

[51] S. Weinberg, The Quantum Theory of Fields III, Cambridge University Press (1996); J. Terning, Modern supersymmetry: Dynamics and duality, Oxford University Press (2006).

[52] C. P. Burgess, R. Kallosh and F. Quevedo, JHEP 0310 (2003) 056, [hep-th/0309187]. A. Saltman and E. Silverstein, JHEP 0411 (2004) 066 [hep-th/0402135].

[53] K. Choi, A. Falkowski, H.P. Nilles and M. Olechowski, Nucl. Phys. B718 (2005) 113 [hep-th/0503216]; S.P. de Alwis, Phys. Lett. B626 (2005) 223 [hep-th/0506266]; G. Villadoro and F. Zwirner, Phys. Rev. Lett. 95 (2005) 231602 [hep-th/0508167]; A. Achucarro, B. de Carlos, J.A. Casas and L. Doplicher, JHEP 0606, 014 (2006) [hep-th/0601190]; G. Villadoro and F. Zwirner, JHEP 0603 (2006) 087 [hep-th/0602120]; Ph. Brax, C. . v. de Bruck, A. C. Davis, S. C. Davis, R. Jeannerot and M. Postma, [hep-th/0610195]; D. Cremades, M. P. Garcia del Moral, F. Quevedo and K. Suruliz, JHEP 0705 (2007) 100 [arXiv:hep-th/0701154]; B. de Carlos, J. A. Casas, A. Guarino, J. M. Moreno and O. Seto, JCAP 0705 (2007) 002 [arXiv:hep-th/0702103].

[54] E. Dudas, C. Papineau and S. Pokorski, JHEP 0702 (2007) 028 [hep-th/0610297]; C.P. Burgess, J.M. Cline, K. das Gupta and H. Firouzjahi, JHEP 0703 (2007) 027 [hep-th/0610320)]; H. Abe, T. Higaki, T. Kobayashi and Y. Omura, Phys. Rev. D 75 (2007) 025019 [hep-th/0611024]; A. Westphal, JHEP 0703 (2007) 102 [hep-th/0611332]; R. Kallosh and M. Soroush, JHEP 0706 (2007) 041 [hep-th/0612057]; S. L. Parameswaran and A. Westphal, Fortsch. Phys. 55 (2007) 804 [hep-th/0701215]; Z. Lalak, O. J. Eyton-Williams and R. Matyszkiewicz, JHEP 0705 (2007) 085 [hep-th/0702026].

[55] J. J. Blanco-Pillado et al., JHEP 0411 (2004) 063 [hep-th/0406230]; Z. Lalak, G. G. Ross and S. Sarkar, [hep-th/0503178]; B. Greene and A. Weltman, [hep-th/0512135].

[56] J. J. Blanco-Pillado et al., JHEP 0609 (2006) 002 [hep-th/0603129].

[57] F. Denef, M. R. Douglas and B. Florea, JHEP 0406 (2004) 034 [hep-th/0404257]; F. Denef, M. R. Douglas, B. Florea, A. Grassi and S. Kachru, [hep-th/0503124].

[58] J. P. Conlon and F. Quevedo, JHEP 0601 (2006) 146 [hep-th/0509012].

[59] J. Simon, R. Jimenez, L. Verde, P. Berglund and V. Balasubramanian, [astro-ph/0605371]; J. R. Bond, L. Kofman, S. Prokushkin and P. M. Vaudrevange, Phys. Rev. D 75 (2007) 123511 [arXiv:hep-th/0612197].

[60] V. Balasubramanian, P. Berglund, J. P. Conlon and F. Quevedo, JHEP 0503 (2005) 007 [hep-th/0502058]; J. P. Conlon, F. Quevedo and K. Suruliz, JHEP 0508 (2005) 007 [hep-th/0505076]; J. P. Conlon, [hep-th/0602233]; J. P. Conlon and F. Quevedo, arXiv:0705.3460 [hep-ph]; M. Berg, M. Haack and E. Pajer, arXiv:0704.0737 [hep-th]. 
[61] C. Herdeiro, S. Hirano and R. Kallosh, JHEP 0112 (2001) 027 [hep-th/0110271]; K. Dasgupta, C. Herdeiro, S. Hirano and R. Kallosh, Phys. Rev. D 65, 126002 (2002) [hep-th/0203019]; J. P. Hsu, R. Kallosh and S. Prokushkin, JCAP 0312 (2003) 009 [hep-th/0311077]; F. Koyama, Y. Tachikawa and T. Watari, [hep-th/0311191]; J. P. Hsu and R. Kallosh, JHEP 0404 (2004) 042 [hep-th/0402047]. K. Dasgupta, J. P. Hsu, R. Kallosh, A. Linde and M. Zagermann, JHEP 0408, 030 (2004) [hep-th/0405247]; P. Chen, K. Dasgupta, K. Narayan, M. Shmakova and M. Zagermann, JHEP 0509, 009 (2005) [hep-th/0501185]; L. McAllister, JCAP 0602 (2006) 010 [hep-th/0502001].

[62] H. Firouzjahi and S. H. H. Tye, Phys. Lett. B 584 (2004) 147 [hep-th/0312020]; S.E. Shandera and S.H. Tye, [hep-th/0601099]. MORE

[63] S. Kachru, R. Kallosh, A. Linde, J. Maldacena, L. McAllister and S. P. Trivedi, JCAP 0310 (2003) 013 [hep-th/0308055].

[64] O. DeWolfe and S. B. Giddings, Phys. Rev. D 67, 066008 (2003) [hep-th/0208123].

[65] D. Baumann, A. Dymarsky, I. R. Klebanov, J. Maldacena, L. McAllister and A. Murugan, [hep-th/0607050].

[66] C. P. Burgess, J. M. Cline, H. Stoica and F. Quevedo, JHEP 0409 (2004) 033 [hep-th/0403119].

[67] J. M. Cline and H. Stoica, Phys. Rev. D 72, 126004 (2005) [hep-th/0508029].

[68] D. Baumann, A. Dymarsky, I. Klebanov, L. McAllister and P. Steinhardt, arXiv:0705.3837 [hep-th]; D. Baumann, A. Dymarsky, I. R. Klebanov and L. McAllister, arXiv:0706.0360 [hep-th]; A. Krause and E. Pajer, arXiv:0705.4682 [hep-th].

[69] S. Sarangi and S. H. H. Tye, Phys. Lett. B 536 (2002) 185 [hep-th/0204074]; T. Matsuda, Phys. Rev. D 70 (2004) 023502 [hep-ph/0403092]; H. Firouzjahi and S. H. Tye, JCAP 0503 (2005) 009 [hep-th/0501099].

[70] E. J. Copeland, R. C. Myers and J. Polchinski, JHEP 0406 (2004) 013 [hep-th/0312067]; G. Dvali, R. Kallosh and A. Van Proeyen, JHEP 0401 (2004) 035 [hep-th/0312005]; G. Dvali and A. Vilenkin, JCAP 0403 (2004) 010 [hep-th/0312007]; L. Leblond and S. H. H. Tye, JHEP 0403 (2004) 055 [hep-th/0402072]; K. Dasgupta, J. P. Hsu, R. Kallosh, A. Linde and M. Zagermann, [hep-th/0405247]; K. Becker, M. Becker and A. Krause, Phys. Rev. D74 (2006) 045023 [hep-th/0510066].

[71] N. Barnaby, C. P. Burgess and J. M. Cline, JCAP 0504 (2005) 007 [hep-th/0412040]; A. R. Frey, A. Mazumdar and R. Myers, Phys. Rev. D 73 (2006) 026003 [hep-th/0508139].

[72] L. Kofman and P. Yi, Phys. Rev. D 72 (2005) 106001 [hep-th/0507257]; D. Chialva, G. Shiu and B. Underwood, JHEP 0601 (2006) 014 [hep-th/0508229]; X. Chen and S. H. Tye, [hep-th/0602136]; P. Langfelder, [hep-th/0602296].

[73] E. Silverstein and D. Tong, from D-cceleration,” Phys. Rev. D 70 (2004) 103505 [hep-th/0310221]; M. Alishahiha, E. Silverstein and D. Tong, Phys. Rev. D 70 (2004) 123505 [hep-th/0404084]; X. g. Chen, JHEP 0508 (2005) 045 [hep-th/0501184]; X. G. Chen, [astro-ph/0507053]; D. Cremades, F. Quevedo and A. Sinha, JHEP 0510 (2005) 106 [hep-th/0505252].

[74] H. V. Peiris, D. Baumann, B. Friedman and A. Cooray, arXiv:0706.1240 [astro-ph].

[75] R. Kallosh and A. Linde, JHEP 0412 (2004) 004 [hep-th/0411011]; J. J. Blanco-Pillado, R. Kallosh and A. Linde, JHEP 0605 (2006) 053 [hep-th/0511042]; R. Kallosh and A. Linde, JHEP 0702 (2007) 002 [hep-th/0611183]. 